TRANSACTIONS OF THE

AMERICAN MATHEMATICAL SOCIETY

Volume 361, Number 7, July 2009, Pages 3535-3596

S 0002-9947(09)04524-3

Article electronically published on March 4, 2009

\title{
CLASSIFICATION OF WEIGHTED DUAL GRAPHS WITH ONLY COMPLETE INTERSECTION SINGULARITIES STRUCTURES
}

\author{
FAN CHUNG, YI-JING XU, AND STEPHEN S.-T. YAU \\ Dedicated to Henry Laufer on the occasion of his 65th birthday
}

\begin{abstract}
Let $p$ be normal singularity of the 2-dimensional Stein space $V$. Let $\pi: M \rightarrow V$ be a minimal good resolution of $V$, such that the irreducible components $A_{i}$ of $A=\pi^{-1}(p)$ are nonsingular and have only normal crossings. Associated to $A$ is weighted dual graph $\Gamma$ which, along with the genera of the $A_{i}$, fully describes the topology and differentiable structure of $A$ and the topological and differentiable nature of the embedding of $A$ in $M$. In this paper we give the complete classification of weighted dual graphs which have only complete intersection singularities but no hypersurface singularities associated to them. We also give the complete classification of weighted dual graphs which have only complete intersection singularities associated with them.
\end{abstract}

\section{INTRODUCTION}

Let $p$ be a normal singularity of the 2-dimensional Stein space $V$. Let $\pi: M \rightarrow$ $V$ be a resolution of $V$ such that the irreducible components $A_{i}, 1 \leq i \leq n$, of $A=\pi^{-1}(p)$ are nonsingular and have only normal crossings. Associated to $A$ is a weighted dual graph $\Gamma$ (e.g., see HNK or La1] which, along with the genera of the $A_{i}$, fully describes the topology and differentiable structure of $A$ and the topological and differentiable nature of the embedding of $A$ in $M$. One of the famous important questions in normal two-dimensional singularities asks: What conditions are imposed on the abstract topology of $(V, p)$ by the complete intersection hypothesis? Recall a theorem of Milnor [Mi, Theorem 2, p. 18] that essentially says that any isolated singularity is a cone over its link $L$ which is the intersection of $V$ with a small sphere centered at $p . \quad L$ is a compact real 3manifold whose oriented homeomorphism type determines and is determined by the weighted dual graph $\Gamma$ of a canonically determined resolution (cf. $[\mathrm{Ne}$ ). So, we may equivalently ask: What conditions will the existence of a complete intersection representative $(V, p)$ put on a weighted dual graph $\Gamma$ ? A complete intersection singularity $(V, p)$ is Gorenstein $\mathrm{Ba}$, Gr-Ri]. So there exists an integral cycle $K$ on $\Gamma$ which satisfies the adjunction formula $[\mathrm{Se}$. The purpose of this paper is to give a complete classification of those weighted dual graphs which have only complete intersection singularities associated to them.

Received by the editors March 2, 2007.

2000 Mathematics Subject Classification. Primary 32S25, 58K65, $14 \mathrm{~B} 05$.

The third author's research was partially supported by an NSF grant. 
M. Artin has studied the rational singularities (those for which $\left.R^{1} \pi_{*}(\mathcal{O})=0\right)$. It is well known that rational complete intersection singularities are hypersurfaces (cf. Theorem 4.3 below). Artin has shown that all hypersurface rational singlarities have multiplicities two and the graphs associated with those singularities are one of the graphs $A_{k}, k \geq 1 ; D_{k}, k \geq 4 ; E_{6}, E_{7}$ and $E_{8}$ which arise in the classification of simple Lie groups. In [La4, Laufer examines a class of elliptic singularities which satisfy a minimality condition. These minimally elliptic singularities have a theory much like the theory for rational singularities. Laufer La4 proved that $p$ is minimally elliptic if and only if $H^{1}(M, \mathcal{O})=\mathbb{C}$ and $\mathcal{O}_{V, p}$ is a Gorenstein ring. Let $Z$ be the fundamental cycle [Ar, p. 132] of the minimal resolution of a minimally elliptic singularity. If $Z^{2}=-1$ or -2 , then $p$ is a double point [La4]. Laufer La4] proved that if $Z^{2}=-3$, then $p$ is a hypersurface singularity with multiplicity 3 . In fact he shows that for a minimally elliptic singularity $Z^{2} \geq-4$ if and only if $p$ is a complete intersection singularity.

Now let $p$ be an arbitrary singularity in the Stein normal 2-dimensional space $V$ having $p$ as its only singularity. Let $\Gamma$ denote the weighted dual graph of the exceptional set of the minimal good resolution $\pi: M \rightarrow V$. In La3, Laufer developed a deformation theory preserving $\Gamma$. This theory allows him to introduce the notion of a property of the associated singularity holding generically for $\Gamma$. Now suppose that $\Gamma$ is a weighted dual graph which does not correspond to a rational double point or to a minimally elliptic singlarity. Then a deep theorm of Laufer [La4] asserts that the corresponding singularity is generically non-Gorenstein. In particular, it is generically not a complete intersection. As a consequence we can characterize those weighted dual graphs which have only complete intersection singularities associated to them. These are precisely rational double point graphs and minimally elliptic graphs with $Z^{2}=-1,-2,-3$ or -4 . Notice that rational double point graphs and minimally elliptic graphs with $Z^{2}=-1,-2$ or -3 are precisely those graphs which have only hypersurface singularities associated with them. Laufer [La4 has completely classified minimally elliptic graphs with $Z^{2}=-1,-2$, or -3 . Therefore in order to classify those weighted dual graphs which have only complete intersection singularities associated with them, we only need to classify all the minimally elliptic graphs with $Z^{2}=-4$. This will be done in section 6 . Incidentally, these graphs are precisely the graphs with complete intersection singularities associated with them but no hypersurface singularities associated with them. We summarize our results in the following theorems.

Theorem A. The complete classification of weighted dual graphs which have only complete intersection singularities but no hypersurface singularities associated to them consists of the minimally elliptic singularity graphs with $Z^{2}=-4$ which are listed in section 7 .

Theorem B. The complete classification of weighted dual graphs which have only complete intersection singularities associated with them consists of rational double point graphs listed in $[\mathrm{Ar}$, minimal elliptic hypersurface singularity graphs listed in [La4] and the minimal elliptic complete intersection singularity graphs listed in section 7 .

Our strategy of classification of all minimally elliptic singularity graphs with $Z^{2}=-4$ is quite simple. We first introduce the concept of an effective component, 
which is an irreducible component $A_{*}$ of the exceptional set such that $A_{*} \cdot Z<0$. It turns out that there are at most 4 effective components with known fundamental coefficients (Proposition 6.2). Let $\Gamma^{\prime}$ be the subgraph of $\Gamma$ obtained by removing all the effective components of $\Gamma$. Suppose $A_{*}$ is an effective component of $\Gamma$. Let $\Gamma_{1}$ be any connected component of $\Gamma^{\prime}$ which intersect with $A_{*}$. Then $\Gamma_{1}$ is necessarily one of the rational double point graphs appearing in Theorem 4.2 . Let $Z_{1}$ be the fundamental cycle of $\Gamma_{1}$. Then $A_{*} \cdot Z_{1} \leq 2$. If $A_{*} \cdot Z_{1}=2$, then $\Gamma=A_{*} \cup \Gamma_{1}$ and $Z=A_{*}+Z_{1}$; moreover for any $A_{j} \in \Gamma_{1}, A_{j} \cdot A_{k}>0$ if and only if $A_{j} \cdot Z_{1}<0$ (Proposition 6.3). In order to find out how one can add $A_{*}$ to the rational double point graphs, we use Theorem 3.5 and the adjunction formula (2.3).

\section{Preliminaries}

Let $\pi: M \rightarrow V$ be a resolution of the normal two-dimensional Stein space $V$. We assume that $p$ is the only singularity of $V$. Let $\pi^{-1}(p)=A=\bigcup A_{i}, 1 \leq i \leq n$, be the decomposition of the exceptional set $A$ into irreducible components.

A cycle $D=\sum d_{i} A_{i}, 1 \leq i \leq n$ is an integral combination of the $A_{i}$, with $d_{i}$ an integer. There is a natural partial ordering, denoted by $<$, between cycles defined by comparing the coefficients. We let supp $D=\bigcup A_{i}, d_{i} \neq 0$, denote the support of $D$.

Let $\mathcal{O}$ be the sheaf of germs of holomorphic functions on $M$. Let $\mathcal{O}(-D)$ be the sheaf of germs of holomorphic functions on $M$ which vanish to order $d_{i}$ on $A_{i}$. Let $\mathcal{O}_{D}$ denote $\mathcal{O} / \mathcal{O}(-D)$. Define

$$
\chi(D):=\operatorname{dim} H^{0}\left(M, \mathcal{O}_{D}\right)-\operatorname{dim} H^{1}\left(M, \mathcal{O}_{D}\right) .
$$

The Riemann-Roch theorem [Se, Proposition IV.4, p. 75] says that

$$
\chi(D)=-\frac{1}{2}\left(D^{2}+D \cdot K\right),
$$

where $K$ is the canonical divisor on $M . D \cdot K$ may be defined as follows. Let $\omega$ be a meromorphic 2-form on $M$. Let $(\omega)$ be the divisor of $\omega$. Then $D \cdot K=D \cdot(\omega)$ and this number is independent of the choice of $\omega$. In fact, let $g_{i}$ be the geometric genus of $A_{i}$, i.e., the genus of the desingularization of $A_{i}$. Then the adjunction formula [Se, Proposition IV, 5, p. 75] says that

$$
A_{i} \cdot K=-A_{i}^{2}+2 g_{i}-2+2 \delta_{i}
$$

where $\delta_{i}$ is the "number" of nodes and cusps on $A_{i}$. Each singular point on $A_{i}$ other than a node or cusp counts as at least two nodes. It follows immediately from (2.2) that if $B$ and $C$ are cycles, then

$$
\chi(B+C)=\chi(B)+\chi(C)-B \cdot C .
$$

Definition 2.1. Associated to $\pi$ is a unique fundamental cycle $Z$ [Ar pp. 131-132] such that $Z>0, A_{i} \cdot Z \leq 0$ for all $A_{i}$ and such that $Z$ is minimal with respect to those two properties. $Z$ may be computed from the intersection as follows via a computation sequence for $Z$ in the sense of Laufer [La2, Proposition 4.1, p. 607]:

$$
\begin{aligned}
Z_{0}=0, Z_{1} & =A_{i_{1}}, Z_{2}=Z_{1}+A_{i_{2}}, \ldots, Z_{j}=Z_{j-1}+A_{i_{j}}, \ldots, \\
Z_{\ell} & =Z_{\ell-1}+A_{i_{\ell}}=Z
\end{aligned}
$$

where $A_{i_{1}}$ is arbitrary and $A_{i_{j}} \cdot Z_{j-1}>0,1<j \leq \ell$. 
$\mathcal{O}\left(-Z_{j-1}\right) /\left(\mathcal{O}\left(-Z_{j}\right)\right.$ represents the sheaf of germs of sections of a line bundle over $A_{i_{j}}$ of Chern class $-A_{i_{j}} \cdot Z_{j-1}$. So

$$
H^{0}\left(M, \mathcal{O}\left(-Z_{j-1}\right) / \mathcal{O}\left(-Z_{j}\right)\right)=0
$$

for $j>1$ and

$$
0 \rightarrow \mathcal{O}\left(-Z_{j-1}\right) / \mathcal{O}\left(-Z_{j}\right) \rightarrow \mathcal{O}_{Z_{j}} \rightarrow \mathcal{O}_{Z_{j-1}} \rightarrow 0
$$

is an exact sheaf sequence. From the long exact cohomology sequence for (2.5), it follows by induction that

$$
\begin{gathered}
H^{0}\left(M, \mathcal{O}_{Z_{k}}\right)=\mathbb{C}, \quad 1 \leq k \leq \ell, \\
\left.\operatorname{dim} H^{1}\left(M, \mathcal{O}_{Z_{k}}\right)=\sum \operatorname{dim}^{1}(M, \mathcal{O})\left(-Z_{j-1}\right) / \mathcal{O}\left(-Z_{j}\right)\right), \\
1 \leq j \leq k .
\end{gathered}
$$

Lemma 2.2 (La4). Let $Z_{k}$ be part of a computation sequence for $Z$ and such that $\chi\left(Z_{k}\right)=0$. Then $\operatorname{dim} H^{1}\left(M, \mathcal{O}_{D}\right) \leq 1$ for all cycles $D$ such that $0 \leq D \leq Z_{k}$. Also $\chi(D) \geq 0$.

\section{Minimally ElLiptic Singularities}

In this section we shall recall some of the properties of minimally elliptic singularities which we need for our classification problem.

Definition 3.1. A cycle $E>0$ is minimally elliptic if $\chi(E)=0$ and $\chi(D)>0$ for all cycles $D$ such that $0<D<E$.

Wagreich Wa defined the singularity $p$ to be elliptic if $\chi(D) \geq 0$ for all cycles $D \geq 0$ and $\chi(F)=0$ for some cycles $F>0$. He proved that this definition is independent of the resolution. It is easy to see that under this hypothesis, $\chi(Z)=0$. The converse is also true La4. Henceforth, we shall adopt the following definition.

Definition 3.2. $p$ is said to be weakly elliptic if $\chi(Z)=0$.

The following proposition and lemma hold for a weakly elliptic singularity.

Proposition 3.3 (La4). Suppose that $\chi(D) \geq 0$ for all cycles $D>0$. Let $B=$ $\sum b_{i} A_{i}$ and $C=\sum c_{i} A_{i}, 1 \leq i \leq n$, be any cycles such that $0<B, C$ and $\chi(B)=\chi(C)=0$. Let $F=\sum \min \left(b_{i}, c_{i}\right) A_{i}, 1 \leq i \leq n$. Then $F>0$ and $\chi(F)=0$. In particular, there exists a unique minimally elliptic cycle $E$.

Lemma 3.4 ([La4]). Let $E$ be a minimally elliptic cycle. Then for $A_{i} \subset \operatorname{supp} E$, $A_{i} \cdot E=-A_{i} \cdot K$. Suppose additionally that $\pi$ is the minimal resolution. Then $E$ is the fundamental cycle for the singularity having supp $E$ as its exceptional set. Also, if $E_{k}$ is part of a computation sequence for $E$ as a fundamental cycle and $A_{j} \subset \operatorname{supp}\left(E-E_{k}\right)$, then the computation sequence may be continued past $E_{k}$ so as to terminate at $E=E_{\ell}$ with $A_{i_{\ell}}=A_{j}$.

Theorem 3.5 ([La4]). Let $\pi: M \rightarrow V$ be the minimal resolution of the normal two-dimensional variety $V$ with one singular point $p$. Let $Z$ be the fundamental cycle on the exceptional set $A=\pi^{-1}(p)$. Then the following are equivalent:

(1) $Z$ is a minimally elliptic cycle,

(2) $A_{i} \cdot Z=-A_{i} \cdot K$ for all irreducible components $A_{i}$ in $A$,

(3) $\chi(Z)=0$ and any connected proper subvariety of $A$ is the exceptional set for a rational singularity. 
In [La4, Laufer introduced the notion of minimally elliptic singularity.

Definition 3.6. Let $p$ be a normal two-dimensional singularity. $p$ is minimally elliptic if the minimal resolution $\pi: M \rightarrow V$ of a neighborhood of $p$ satisfies one of the conditions of Theorem 3.5 .

Proposition 3.7 ([La4]). Let $\pi: M \rightarrow V$ and $\pi^{\prime}: M^{\prime} \rightarrow V$ be the minimal resolution and minimal good resolution respectively for a minimally elliptic singularity $p$. Then $\pi=\pi^{\prime}$ and all the $A_{i}$ are rational curves except for the following cases:

(1) $A$ is an elliptic curve. $\pi$ is a minimal good resolution.

(2) A is a rational curve with a node singularity.

(3) $A$ is a rational curve with a cusp singularity.

(4) A is two nonsingular rational curves which have first order tangential contact at one point.

(5) $A$ is three nonsingular rational curves all meeting transversely at the same point.

In case (2), the weighted dual graph of the minimal good resolution is

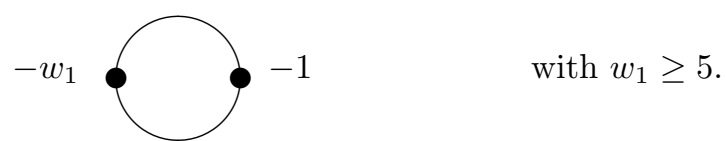

In cases (3)-(5), $\pi^{\prime}$ has the following weighted dual graph:

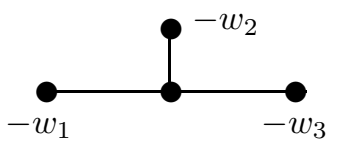

with $w_{i} \geq 2,1 \leq i \leq 3$.

Minimally elliptic singularities can be characterized without explicit use of the resolution as follows because $H^{1}(M, \mathcal{O})$ can be described in terms of $V$ La2, Theorem 3.4, p. 604].

Theorem 3.8 (La4). Let $V$ be a Stein normal two-dimensional space with $p$ as its only singularity. Let $\pi: M \rightarrow V$ be a resolution of $V$. Then $p$ is a minimally elliptic singularity if and only if $H^{1}(M, \mathcal{O})=\mathbb{C}$ and $\mathcal{O}_{V, p}$ is a Gorenstein ring.

\section{Weighted DUAL GRAPHS ADMITTING NO COMPLETE INTERSECTION SINGULARITIES STRUCTURES}

In this section, we shall show that there is a large class of weighted dual graphs not admitting any complete intersection singularity structure. Let $(V, p)$ be a normal 2-dimensional singularity. Let $\pi: M \rightarrow V$ be the minimal resolution. Let $Z$ be the fundamental cycle.

Definition 4.1. $p$ is a rational singularity if $\chi(Z)=1$.

If $p$ is a rational singularity, then $\pi$ is also a minimal good resolution, i.e., exceptional set with nonsingular $A_{i}$ and normal crossings. Moreover each $A_{i}$ is a rational curve $\mathrm{Ar}$. 
Theorem $4.2(\underline{\mathrm{Ar}})$. If $p$ is a hypersurface rational singularity, then $p$ is a rational double point. Moreover the set of weighted dual graphs of hypersurface rational singularities consists of the following graphs:
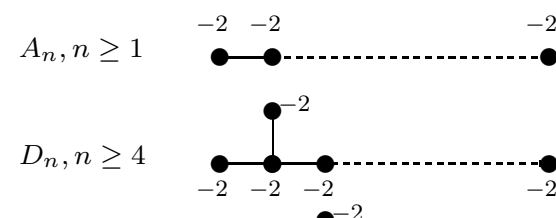

$E_{6}$

(4) $\quad E_{7}$

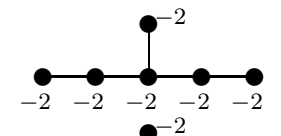

(5) $E_{8}$

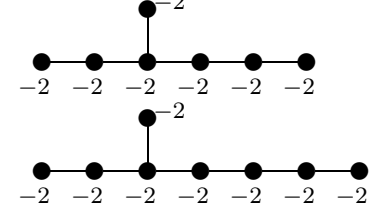

Theorem 4.3. Let $\Gamma$ be a weighted dual graph of a rational singularity. If $\Gamma$ is not one of the five types in Theorem 4.2, then $\Gamma$ does not admit any Gorenstein singularity structure; in particular, $\Gamma$ does not admit any complete intersection singularity structure.

Proof. Since in the definition of a rational singularity, $\chi(Z)$ can be computed from the weighted dual graph, any singularity associated to $\Gamma$ is a rational singularity. To prove the theorem, we only need to prove that if $p$ is a Gorenstein rational singularity, then its graph is one of the five types in Theorem 4.2 Suppose $(V, p)$ is a Gorenstein rational singularity. Then $\operatorname{dim} H^{1}(M, \mathcal{O})=0$ Ar. By a result of Laufer [La2], $\operatorname{dim} H^{1}(M, \mathcal{O})=\operatorname{dim} H^{0}\left(M-A, \Omega^{2}\right) / H^{0}\left(M, \Omega^{2}\right)$ where $\Omega^{2}$ is the sheaf of germs of holomorphic 2-forms on $M$. Therefore there exists an effective canonical divisor $K=\sum k_{i} A_{i}, k_{i}$ a nonnegative integer, on $M$. Since $M$ is a minimal resolution, by the adjunction formula, we have

$$
A_{i} \cdot K \geq 0 \text { for all } A_{i} \subseteq A \text {. }
$$

It follows that

$$
K^{2}=\sum k_{i}\left(A_{i} \cdot K\right) \geq 0
$$

On the other hand, the intersection matrix is a negative definition $\mathrm{Gr}$. Therefore $K^{2} \leq 0$. This together with (4.2) implies $K^{2}=0$. The negative definiteness of the intersection matrix implies $K=0$. The adjunction formula tells us that $A_{i}^{2}=-2$ for all $A_{i}$. Then as an easy exercise, one can show that the weighted dual graph of the exceptional set is one of the five types listed in Theorem 4.2 .

\section{Characterization of Weighted DuAl Graphs admitting only COMPLETE INTERSECTION SINGULARITIES STRUCTURES}

In this section we shall give a characterization of weighted dual graphs admitting only hypersurface singularities structures. We shall also give a characterization of weighted dual graphs admitting only complete intersection singularities structures. 
It turns out that the latter list minus the former list corresponds to the list of weighted dual graphs which admit only complete intersection singularities structures but not hypersurface singularities structures.

Theorem 5.1 ([La4]). Let $p$ be a minimally elliptic singularity. Let $\pi: M \rightarrow V$ be a resolution of a Stein neighborhood $V$ of $p$ with $p$ as its only singular point. Let $m$ be the maximal ideal in $\mathcal{O}_{V, p}$. Let $Z$ be the fundamental cycle on $A=\pi^{-1}(p)$.

(1) If $Z^{2} \leq-2$, then $\mathcal{O}(-Z)=m \mathcal{O}$ on $A$.

(2) If $Z^{2}=-1$, and $\pi$ is the minimal resolution or the minimal resolution with nonsingular $A_{i}$ and normal crossings, then $\mathcal{O}(-Z) / m \mathcal{O}$ is the structure sheaf for an embedded point.

(3) If $Z^{2}=-1$ or -2 , then $p$ is a double point.

(4) If $Z^{2}=-3$, then for all integers $n \geq 1, m^{n} \approx H^{0}(A, \mathcal{O}(-n Z)$ ) and $\operatorname{dim} m^{n} / m^{n+1}=-n Z^{2}$.

(5) If $-3 \leq Z^{2} \leq-1$, then $p$ is a hypersurface singularity.

(6) If $Z^{2}=-4$, then $p$ is a complete intersection and in fact a tangential complete intersection.

(7) If $Z^{2} \leq-5$, then $p$ is not a complete intersection.

Let $p$ be a normal two-dimensional singularity. Choose the minimal resolution of $p$ having nonsingular $A_{i}$ and normal crossings. Let $\Gamma$ denote the weighted dual graph along with the genera. See [HNK] or [La1 for a more detailed description of $\Gamma$. $\Gamma$ may be described abstractly. Given $\Gamma$, we say that $p$ is a singularity associated to $\Gamma$. As in [La1, Theorem 6.20, p. 132] we may choose a suitably large infinitesimal neighborhood $B$ of the exceptional set such that $B$ depends only on $\Gamma$ and determines $p$. We can deform $B$ in such a way that $\Gamma$ is preserved. See La3] for the general theory in this situation.

Definition 5.2. Let $\Gamma$ be a weighted dual graph, including genera for the vertices. A property is generically true for an associated singularity of $\Gamma$ if given any normal two-dimensional singularity $p$ having $\Gamma$ as the weighted dual graph of its minimal resolution with nonsingular $A_{i}$ and normal crossings, then the property is true for all singularities near $p$ and off a proper subvariety of the parameter space of a complete deformation of a suitable large infinitesimal neighborhood $B$ of the exceptional set for $P$.

The following deep theorem is due to Laufer.

Theorem 5.3 ([La4]). All rational double points and all minimally elliptic singularities are Gorenstein. Let $\Gamma$ be a weighted dual graph, including genera for the vertices, associated to a minimal resolution with nonsingular $A_{i}$ and normal crossings of a singularity $p$. Suppose that $p$ is not a rational double point or minimally elliptic. Then an associated singularity of $\Gamma$ is generically non-Gorenstein.

Now we are ready to give a characterization of weighted dual graphs admitting only complete intersection singularities structures (respectively hypersurface singularities structures). Recall that rational and minimally elliptic singularities have topological definitions; i.e., they can be defined in terms of their weighted dual graphs. 


\section{Theorem 5.4.}

(1) The weighted dual graphs which have only hypersurface singularities associated to them are precisely those graphs coming from rational double points, minimally elliptic double points $\left(Z^{2}=-1\right.$, or -2$)$, or minimally elliptic triple points $\left(Z^{2}=-3\right)$.

(2) The weighted dual graphs which have only complete intersection singularities associated to them are precisely those graphs coming from rational double points, minimally elliptic double points $\left(Z^{2}=-1\right.$, or -2$)$, minimally elliptic triple points $\left(Z^{2}=-3\right)$, or minimally elliptic quadruple points $\left(Z^{2}=-4\right)$.

(3) The weighted dual graphs which have only complete intersection but not hypersurface singularities associated to them are precisely those graphs coming from minimally elliptic quadruple points $\left(Z^{2}=-4\right)$.

Proof. We only need to observe that hypersurface or complete intersection singularities are Gorenstein. Theorem $[5.4$ follows directly from Theorem 5.1 and Theorem 5.3 .

\section{Classification of Weighted dual Graphs With only Complete INTERSECTION BUT NOT HYPERSURFACE SINGULARITIES STRUCTURES}

By Theorem 5.4 the classification of weighted dual graphs with only complete intersection but not hypersurface singularity structure is equal to the classification of minimally elliptic singularity weighted dual graphs with $Z^{2}=-4$.

Definition 6.1. Let $(V, p)$ be a germ of weakly elliptic singularity. Let $\pi: M \rightarrow V$ be the minimal resolution with $\pi^{-1}(p)=A=\bigcup A_{i}, 1 \leq i \leq n$ the irreducible decomposition of the exceptional set. Let $Z$ be the fundamental cycle. The set of effective components $\left\{A_{* 1}, \ldots, A_{* n}\right\}$ is the set $\left\{A_{i}: A_{i} \cdot Z<0\right\}$.

Proposition 6.2. Let $(V, p)$ be a germ of minimally elliptic singularity. Let $\pi$ : $M \rightarrow V$ be the minimal resolution of $p$. If $\pi$ is also a minimal good resolution and $Z^{2}=-4$, then the set of effective components $\left\{A_{* 1}, \ldots, A_{* m}\right\}$ must be one of the following:

(1) $\left\{A_{* 1}\right\}, A_{* 1}^{2}=-3, z_{1}=4$

(2) $\left\{A_{* 1}\right\}, A_{* 1}^{2}=-4, z_{1}=2$

(3) $\left\{A_{* 1}\right\}, A_{* 1}^{2}=-6, z_{1}=1$

(4) $\left\{A_{* 1}, A_{* 2}\right\}, A_{* 1}^{2}=A_{* 2}^{2}=-3, z_{1}=z_{2}=2$

(5) $\left\{A_{* 1}, A_{* 2}\right\}, A_{* 1}^{2}=A_{* 2}^{2}=-3, z_{1}=3, z_{2}=1$

(6) $\left\{A_{* 1}, A_{* 2}\right\}, A_{* 1}^{2}=-3, A_{* 2}^{2}=-4, z_{1}=2, z_{2}=1$

(7) $\left\{A_{* 1}, A_{* 2}\right\}, A_{* 1}^{2}=A_{* 2}^{2}=-4, z_{1}=z_{2}=1$

(8) $\left\{A_{* 1}, A_{* 2}\right\}, A_{* 1}^{2}=-3, A_{* 2}^{2}=-5, z_{1}=z_{2}=1$

(9) $\left\{A_{* 1}, A_{* 2}, A_{* 3}\right\}, A_{* 1}^{2}=A_{* 2}^{2}=A_{* 3}^{2}=-3, z_{1}=z_{2}=1, z_{3}=2$

(10) $\left\{A_{* 1}, A_{* 2}, A_{* 3}\right\}, A_{* 1}^{2}=A_{* 2}^{2}=-3, A_{* 3}^{2}=-4, z_{1}=z_{2}=z_{3}=1$

(11) $\left\{A_{* 1}, A_{* 2}, A_{* 3}, A_{* 4}\right\}, A_{* 1}^{2}=-3, z_{i}=1, i=1,2,3,4$,

where $A_{* i} \neq A_{* j}$ if $i \neq j$ and $z_{i}$ is the coefficient of $A_{* i}$ in $Z$. 
Proof. Let $\left\{A_{* 1}, \ldots, A_{* m}\right\}$ be the set of effective components. Then, by Theorem 3.5 we have

$$
\begin{aligned}
-Z^{2} & =-\sum_{i=1}^{n} z_{i}\left(A_{i} \cdot Z\right)=-\sum_{i=1}^{m} z_{i}\left(A_{* i} \cdot Z\right) \\
& =\sum_{i=1}^{m} z_{i}\left(A_{* i} \cdot K\right) .
\end{aligned}
$$

This implies that $4=\sum_{i=1}^{m} z_{i}\left(-A_{* i}^{2}-2\right)$. By the definition of the effective component, we have $-A_{* i}^{2}-2=A_{* i} \cdot K=-A_{* i} \cdot Z>0$. Hence we have $1 \leq$ $m \leq 4$. If $m=1$, then $-4=z_{1}\left(A_{* 1}^{2}+2\right)$ and we are in case (1), case (2) or case (3). If $m=2$, then $-4=z_{1}\left(A_{* 1}^{2}+2\right)+z_{2}\left(A_{* 2}^{2}+2\right)$. It follows easily that we are in case (4), case (5), case (6), case (7), or case (8). If $m=3$, then $-4=z_{1}\left(A_{* 1}^{2}+2\right)+z_{2}\left(A_{* 2}^{2}+2\right)+z_{3}\left(A_{* 3}^{2}+2\right)$. It is easy to see that we are in case (9) or case (10). If $m=4$, then $-4=\left(A_{* 1}^{2}+2\right)+\left(A_{* 2}^{2}+2\right)+\left(A_{* 3}^{2}+2\right)+\left(A_{* 4}^{2}+2\right)$. So we are in case (11).

Proposition 6.3. Let $\Gamma$ be the minimal resolution graph of a minimally elliptic singularity with fundamental cycle $Z$. Let $\Gamma^{\prime}$ be the subgraph of $\Gamma$ obtained by removing all the effective components of $\Gamma$. Suppose that $A_{*}$ is an effective component of $\Gamma$, and let $\left\{\Gamma_{1}, \ldots, \Gamma_{n}\right\}$ be the set of connected components of $\Gamma^{\prime}$ which intersect with $A_{*}$. Then $\Gamma_{1}, \ldots, \Gamma_{n}$ are necessarily one of the rational double point graphs appearing in Theorem 4.2. Let $Z_{1}, \ldots, Z_{n}$ be the fundamental cycles of $\Gamma_{1}, \ldots, \Gamma_{n}$ respectively. Then $A_{*} \cdot Z_{1} \leq 2$. If $A_{*} \cdot Z_{1}=2$, then $\Gamma=A_{*} \cup \Gamma_{1}$ and $Z=A_{*}+Z_{1}$; moreover for any $A_{1} \in \Gamma_{1}, A_{1} \cdot A_{*}>0$ if and only if $A_{1} \cdot Z_{1}<0$.

Proof. For any $A_{j} \in \Gamma_{i}, 0=A_{j} \cdot Z=A_{j} \cdot(-K)=A_{j}^{2}+2$. Hence $A_{j}^{2}=-2$. It follows that $\Gamma_{i}$ are rational double point graphs.

Since $\Gamma$ is the graph of a minimally elliptic singularity, we have

$$
\begin{aligned}
0 & \leq \chi\left(A_{*}+Z_{1}\right) \\
& =\chi\left(A_{*}\right)+\chi\left(Z_{1}\right)-A_{*} \cdot Z_{1},
\end{aligned}
$$

which implies

$$
A_{*} \cdot Z_{1} \leq \chi\left(A_{*}\right)+\chi\left(Z_{1}\right)=2 .
$$

Observe that if $\Gamma \neq A_{*} \cup \Gamma_{1}$ or $Z>A_{*}+Z_{1}$, then the inequalities in (6.1) and (6.2) are strict inequalities. Hence $A_{*} \cdot Z_{1}=1$. We have proved that if $A_{*} \cdot Z_{1}=2$, then $\Gamma=A_{*} \cup \Gamma_{1}$ and $Z=A_{*}+Z_{1}$.

We shall assume from now on that $A_{*} \cdot Z_{1}=2$. Let $A_{1} \in \Gamma_{1}$ such that $A_{1} \cdot A_{*}>0$. $A_{1} \cdot Z_{1}=0$ would imply $A_{1} \cdot\left(Z_{1}+A_{*}\right)>0$ and hence $A_{1} \cdot Z>0$, which is absurd. It follows that $A_{1} \cdot Z_{1}<0$.

Conversely, if $A_{1} \in \Gamma_{1}$ and $A_{1} \cdot Z_{1}<0$, but $A_{*} \cdot A_{1}=0$, then there is an $A_{2} \in \Gamma_{1}$ such that $A_{2} \cdot A_{*}>0$ and $A_{2} \cdot Z_{1}<0$. Since $Z_{1}^{2}=-2$, we have $A_{2} \cdot Z_{1}=A_{1} \cdot Z_{1}=-1$ and the coefficient of $A_{2}$ in $Z_{1}$ is one. It follows that $A_{2}$ is the only component in $\Gamma_{1}$ which intersects with $A_{*}$ and $A_{2} \cdot A_{*}=2$. Observe that $\chi\left(A_{*}+A_{2}\right)=0$ and $A_{*}+A_{2}<Z$. This contradicts the fact that $Z$ is the minimally elliptic cycle. So we have shown that $A_{*} \cdot A_{1}>0$ if and only if $A_{1} \cdot Z_{1}<0$. 
Notation. From now on, we shall denote $\bullet$ a nonsingular rational curve with -2 weight.

Corollary 6.4. Let $\Gamma$ be the minimal resolution graph of a minimally elliptic singularity with fundamental cycle $Z$. Let $\Gamma_{1}$ be a rational double point subgraph of $\Gamma$ with fundamental cycle $Z_{1}$ in Proposition 6.3. Let $A_{*}$ be an effective component attaching on $\Gamma_{1}$. Suppose that $A_{*} \cdot Z_{1}=2$. Then one of the following cases holds.

(1) $\Gamma$ is of the following form:

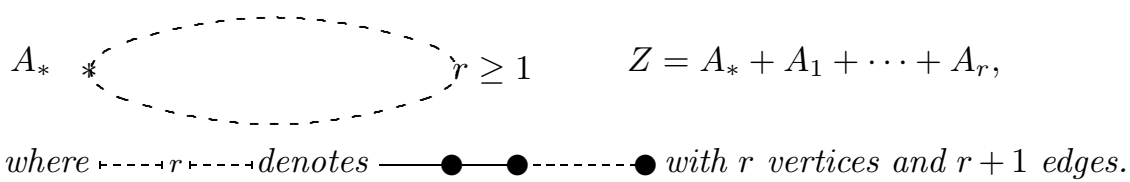

- is a nonsingular rational curve with weight -2 .

(2) $\Gamma_{1}$ is either $D_{n}, E_{6}, E_{7}$ or $E_{8}$. There exists a unique $A_{1}$ in $\Gamma_{1}$ such that $A_{1} \cdot A_{*}=1$ and $A_{1} \cdot Z_{1}<0$. The coefficient of $A_{1}$ in $Z_{1}$ is 2 . $\Gamma=A_{*} \cup \Gamma_{1}$ and $Z=A_{*}+Z_{1} . \Gamma$ is one of the following forms.

(a) (i)

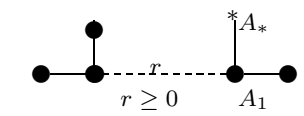

$$
\begin{aligned}
& Z=1 \quad \begin{array}{rrrr}
1 & & 1 \\
& 2 & 2 & 2
\end{array} \\
& Z=1 \begin{array}{ll}
1 \\
2 & 1 \\
1
\end{array}
\end{aligned}
$$

(ii)

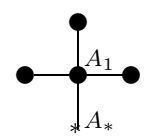

(b)
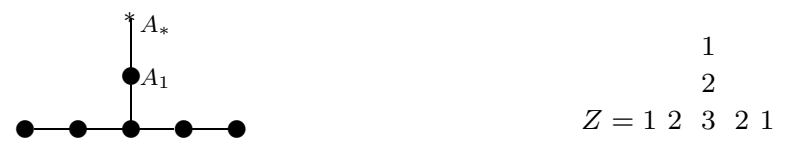

(c)

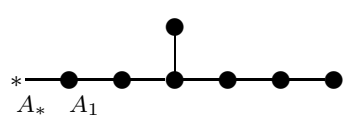

$Z=1223 \quad 4 \quad 3221$

(d)

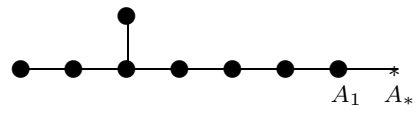

3

$Z=24 \quad 6 \quad 543221$

Proof. This follows from Proposition 6.3 and Theorem 4.2 .

Definition 6.5. Let $A_{1}$ be an irreducible component in a weighted dual graph $\Gamma$. The degree of $A_{1}$ is defined to be the number of distinct irreducible components in $\Gamma$ intersecting with $A_{1}$ positively.

Lemma 6.6. Let $\Gamma$ be the minimal resolution graph of a minimally elliptic singularity with fundamental cycle $Z$. Let $\Gamma_{1}$ be a subgraph of $\Gamma$ in Proposition 6.3 with fundamental cycle $Z_{1}$. Let $A_{*}$ be an effective component attaching on $\Gamma_{1}$. Suppose 
that the coefficient $z_{*}$ of $A_{*}$ in $Z$ is one. Then either $A_{*}$ has degree one or $\Gamma$ is of the following form:

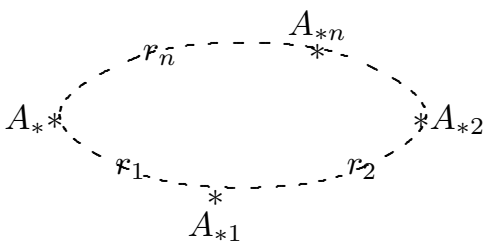

where $n \geq 1$ and $\Gamma_{1}$ is $\ldots-\ldots r+--$ which denotes $\longrightarrow-\bullet-\ldots$ with $r_{1}$ vertices and $r_{1}+1$ edges.

Proof. By Proposition 6.3, $A_{*} \cdot Z_{1}$ is either 1 or 2 . If $A_{*} \cdot Z_{1}=2$, then the lemma follows from Corollary 6.4.

From now on, we shall assume that $A_{*} \cdot Z_{1}=1$. To prove the lemma, we only need to prove that if $\operatorname{deg} A_{*}>1$, then $\Gamma$ must be of the circular form shown as above. If $\operatorname{deg} A_{*}>1$, then there exists $A_{2}$ not in $\Gamma_{1}$ such that $A_{2} \cdot A_{*}>0$. Clearly $A_{2} \cdot A_{*}=1$ by the minimal ellipticity of $\Gamma$. We claim that $A_{2}$ is connected to $\Gamma_{1}$ via a path in $\Gamma$ which is disjoint from $A_{*}$.

By Theorem 3.4 we can choose a computation sequence of the fundamental cycle $Z$ starting from $A_{*}$ continuing to $\Gamma_{1}$ and ending at $A_{2}$. Now $z_{*}=1, A_{*}^{2}+2=A_{*} \cdot Z$, and $\operatorname{deg} A_{*}>1$ implies that the computation sequence contains $A_{*}$ only once and the coefficient of $A_{2}$ in $Z$ must also be one. Hence the computation sequence must contain $A_{2}$ only once. Moreover $A_{2}^{2}+2=A_{2} \cdot Z$ implies that $\operatorname{deg} A_{2}=2$. Repeating the same argument, we see that for every component in that computation sequence its coefficient in $Z$ is one, its degree is 2 and the computation sequence passes it only once. Therefore $\Gamma$ must be the form shown in the lemma.

Remark 6.7. With the same assumption and notation as in Lemma 6.6, so long as the interseciton matrix remains negative definite, $A_{*}^{2}$ can be given any value at most -2 and $Z$ remains unchanged and $\Gamma$ still corresponds to a minimally elliptic singularity.

Proposition 6.8. Let $\Gamma$ be the minimal resolution graph of minimally elliptic singularity with fundamental cycle $Z$. Suppose that there is no effective component with coefficient in $Z$ strictly greater than 1 . Set all $A_{*}^{2}$ of effective components of $\Gamma$ but one to -2 and the remaining weight to -3 . Then the new weighted dual graph $\widetilde{\Gamma}$, which coincides with $\Gamma$ except for the weights, is obtained from a rational double point weighted dual graph by the addition of one additional vertex $A_{*}$. In fact $\widetilde{\Gamma}$ corresponds to a minimally elliptic double point with $Z^{2}=-1$.

Proof. Since $A_{*} \cdot Z=-A_{*} \cdot K=A_{*}^{2}+2$, after setting all $A_{*}^{2}$ of effective components of $\Gamma$ but one to -2 and the remaining weight to -3 , it is still true that $A_{i} \cdot Z \leq 0$ for all $i$ and that $A_{*} Z<0$ for one $A_{*}$. Therefore $Z$ is also the fundamental cycle for $\widetilde{\Gamma}$ and the intersection matrix of $\widetilde{\Gamma}$ is still negative definite $\mathrm{Ar}$, Proposition 2, pp. 130-131]. By Lemma 6.6. $\Gamma$ is obtained from a rational double point weighted dual graph by the addition of one additional vertex $A_{*}$. Clearly $Z_{\widetilde{\Gamma}}^{2}=-1$. 
Proposition 6.9. Let $\Gamma$ be the minimal resolution graph of a minimally elliptic singularity with fundamental cycle $Z$. Let $\Gamma^{\prime}$ be the subgraph of $\Gamma$ obtained by removing all the effective components of $\Gamma$. Let $A_{*}$ be an effective component of $\Gamma$. Suppose that $\Gamma_{1}$ is a connected component of $\Gamma^{\prime}$ which corresponds to the $A_{n}$ graph in case (1) of Theorem 4.2. Suppose also that $\Gamma_{1}$ intersects with $A_{*}$ but is disjoint from other effective components. Let $Z_{1}$ be the fundamental cycle on $\Gamma_{1}$. Suppose $A_{*} \cdot Z_{1}=1$. If the coefficient $z_{*}$ of $A_{*}$ in $Z$ is four and $A_{*}^{2}=-3$, then $A_{*} \cup \Gamma_{1}$ and the restriction of $Z$ on $A_{*} \cup \Gamma_{1}$ must be one of the following forms.

(1)

$A_{*}$

(2)

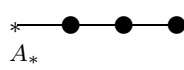

(3)

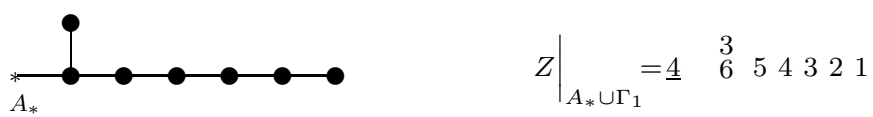

(4)

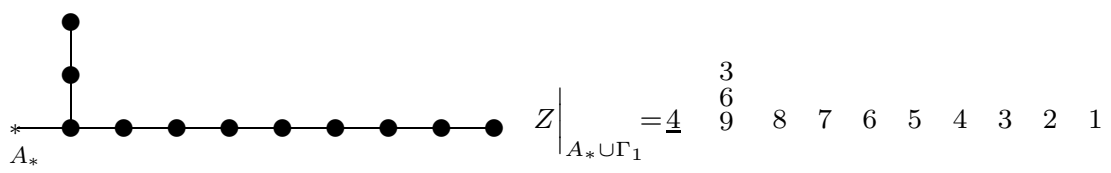

(5)

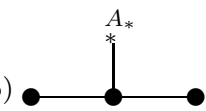

(6)
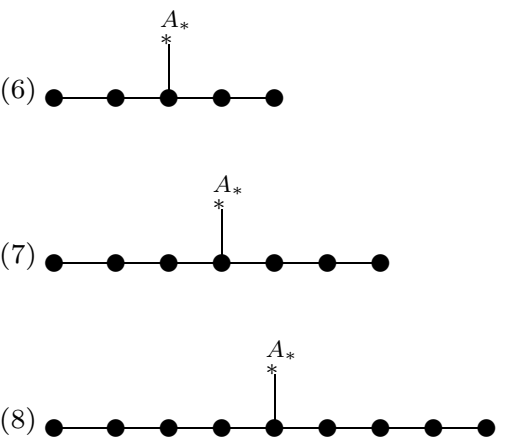

$\left.Z\right|_{A_{*} \cup \Gamma_{1}}=\underline{4} \quad 2$

$\left.Z\right|_{A_{*} \cup \Gamma_{1}}=\underline{4} \quad 3 \quad 2 \quad 1$

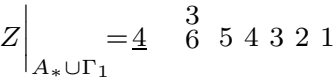

$\left.Z\right|_{A_{*} \cup \Gamma_{1}}=2 \quad \frac{4}{4} \quad 2$

$\left.Z\right|_{A_{*} \cup \Gamma_{1}}=2 \quad 4 \quad \frac{4}{6} \quad 4 \quad 2$

$\left.Z\right|_{A_{*} \cup \Gamma_{1}}=2 \quad 4 \quad 6 \quad \frac{4}{8} \quad 6 \quad 4 \quad 2$

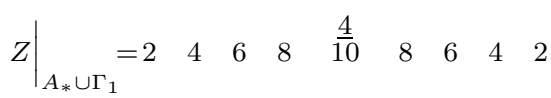

Proof. Consider $A_{*}$ attaching on $\Gamma_{1}$ in the following form:

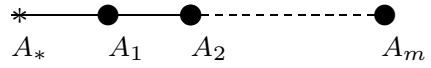

$$
\left.Z\right|_{A_{*} \cup \Gamma_{1}}=4 \quad n_{1} \quad n_{2} \ldots n_{m}
$$


Since $A_{i} \cdot Z=A_{i} \cdot(-K)=A_{i}^{2}+2=0,1 \leq i \leq m$, we have the following system of equations:

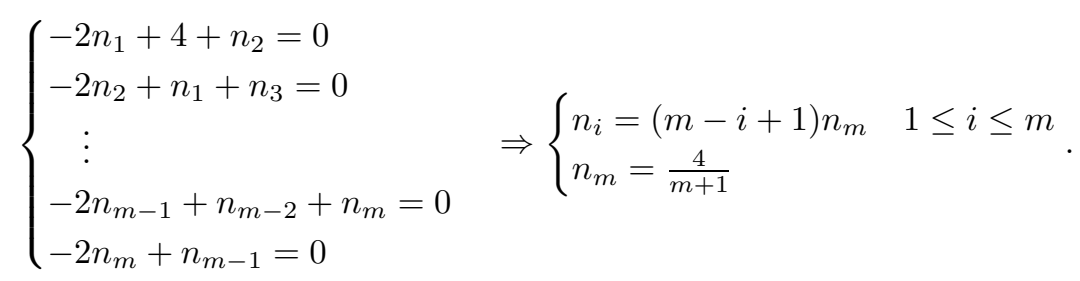

Therefore $m=1$ or $m=3$ and we are in case (1) or case (2) respectively.

Consider $A_{*}$ attaching on $\Gamma_{1}$ in the following form:

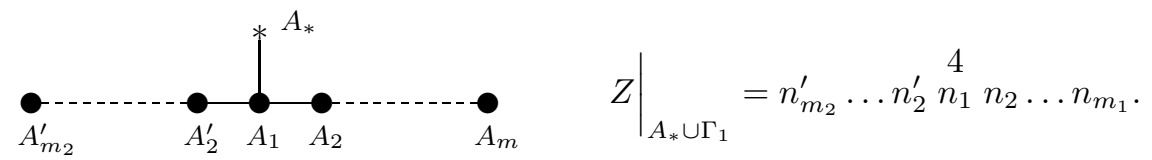

Since $A_{i} \cdot Z=A_{i} \cdot(-K)=A_{i}^{2}+2=0,1 \leq i \leq m_{1}$ and similarly $A_{j}^{\prime} \cdot Z=0$ for $2 \leq j \leq m_{2}^{\prime}$, we have the following system of equations:

$$
\left\{\begin{array} { l } 
{ - 2 n _ { m _ { 1 } } + n _ { m _ { 1 } - 1 } = 0 } \\
{ - 2 n _ { m _ { 1 } - 1 } + n _ { m _ { 1 } - 2 } + n _ { m _ { 1 } } = 0 } \\
{ \vdots } \\
{ - 2 n _ { 3 } + n _ { 2 } + n _ { 4 } = 0 } \\
{ - 2 n _ { 2 } + n _ { 1 } + n _ { 3 } = 0 }
\end{array} \quad \left\{\begin{array}{l}
-2 n_{m_{2}}^{\prime}+n_{m_{2}-1}^{\prime}=0 \\
-2 n_{m_{2}-1}^{\prime}+n_{m_{2}-2}^{\prime}+n_{m_{2}}^{\prime}=0 \\
\vdots \\
-2 n_{3}^{\prime}+n_{2}^{\prime}+n_{4}^{\prime}=0 \\
-2 n_{2}^{\prime}+n_{1}^{\prime}+n_{3}^{\prime}=0
\end{array}\right.\right.
$$

(6.3) implies

$$
\begin{array}{rlrl}
n_{i} & =\left(m_{1}-i+1\right) n_{m_{1}} & & 1 \leq i \leq m_{1}, \\
n_{j}^{\prime} & =\left(m_{2}-j+1\right) n_{m_{2}}^{\prime} & & 2 \leq j \leq m_{2}, \\
m_{1} n_{m_{1}} & =m_{2} n_{m_{2}}^{\prime} .
\end{array}
$$

Putting (6.5) and (6.6) into (6.4), we get

$$
\begin{aligned}
0 & =4-2 m_{1} n_{m_{1}}+\left(m_{1}-1\right) n_{m_{1}}+\left(m_{2}-1\right) n_{m_{2}}^{\prime} \\
& =4-\left(m_{1}+1\right) n_{m_{1}}+\left(m_{2}-1\right) n_{m_{2}}^{\prime}=0 .
\end{aligned}
$$

(6.7) and (6.8) imply

$$
n_{m_{1}}+n_{m_{2}}^{\prime}=4
$$

(6.9) implies that either $n_{m_{1}}=3, n_{m_{2}}^{\prime}=1$ or $n_{m_{1}}=2=n_{m_{2}}^{\prime}$.

Case I. $n_{m_{1}}=3$ and $n_{m_{2}}^{\prime}=1$. By (6.7), we have $3 m_{1}=m_{2}$. Observe that

$$
\begin{aligned}
& -1=A_{*}^{2}+2=A_{*} \cdot(-K)=A_{*} \cdot Z \geq 4(-3)+n_{1}=-12+3 m_{1} \\
\Rightarrow & 3 m_{1} \leq 11 \\
\Rightarrow & m_{1} \leq 3
\end{aligned}
$$


If $m_{1}=1$, or 2 , or 3 , then we are in case (2), case (3) or case (4) respectively in the statement of the proposition.

Case II. $n_{m_{1}}=2=n_{m_{2}}^{\prime}$. By (6.7), we have $m_{1}=m_{2}$. Observe that

$$
\begin{aligned}
& -1=A_{*} \cdot(-K)=A_{*} \cdot Z \geq 4(-3)+n_{1}=-12+2 m_{1} \\
\Rightarrow & 2 m_{1} \leq 11 \\
\Rightarrow & m_{1} \leq 5 .
\end{aligned}
$$

If $m_{1}=2$, or 3 , or 4 , or 5 , then we are in case (5), case (6), case $(7)$ or case $(8)$ respectively in the statement of the proposition.

Proposition 6.10. Let $\Gamma$ be the minimal resolution graph of a minimally elliptic singularity with fundamental cycle $Z$. Let $\Gamma^{\prime}$ be the subgraph of $\Gamma$ obtained by removing all the effective components of $\Gamma$. Let $A_{*}$ be an effective component of $\Gamma$. Suppose that $\Gamma_{1}$ is a connected component of $\Gamma^{\prime}$ which corresponds to the $D_{n}$ graph in case (2) of Theorem 4.2. Suppose also that $\Gamma_{1}$ intersects with $A_{*}$ but is disjoint from other effective components. Let $Z_{1}$ be the fundamental cycle on $\Gamma_{1}$. Suppose $A_{*} \cdot Z_{1}=1$. If the coefficient $z_{*}$ of $A_{*}$ in $Z$ is four and $A_{*}^{2}=-3$, then $A_{*} \cup \Gamma_{1}$ and the restriction of $Z$ on $A_{*} \cup \Gamma_{1}$ must be one of the following forms.

(1)

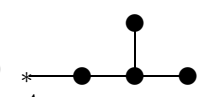

(2)

$A_{*}$

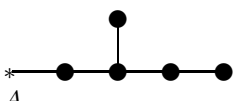

(3)

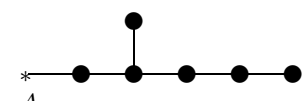

(4)

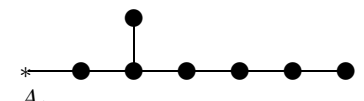

(5)

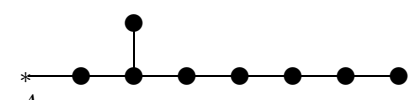

(6)

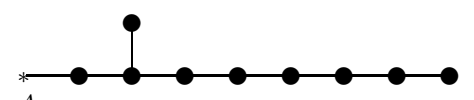

(7)

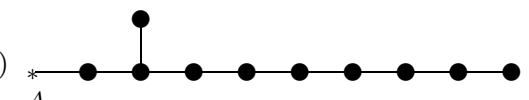

(8)

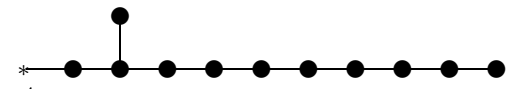

$A_{*}$

(9)

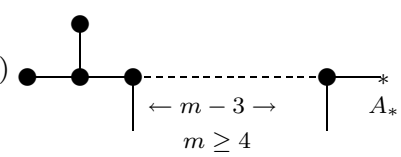

$Z_{A_{*} \cup \Gamma_{1}}=\underline{4} 4 \stackrel{2}{4} 2$

$Z_{A_{*} \cup \Gamma_{1}}=\underline{4} 5 \stackrel{3}{6} \quad 42$

$Z_{A_{*} \cup \Gamma_{1}}=\underline{4} 6 \stackrel{4}{8} \quad 6 \quad 42$

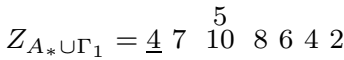

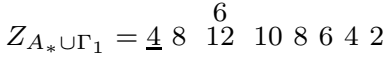

$Z_{A_{*} \cup \Gamma_{1}}=\underline{4} 9 \quad \stackrel{7}{14} 12108642$

$Z_{A_{*} \cup \Gamma_{1}}=\underline{4} 10 \quad \stackrel{8}{16} 141412108642$

$Z_{A_{*} \cup \Gamma_{1}}=\underline{4} 11 \stackrel{9}{9} 18 \quad 161412108642$

$Z_{A_{*} \cup \Gamma_{1}}=2 \stackrel{2}{4} \quad 4 \quad \ldots .4 \quad \underline{4}$ 
Proof. Consider $A_{*}$ attaching on $\Gamma_{1}$ in the following form:

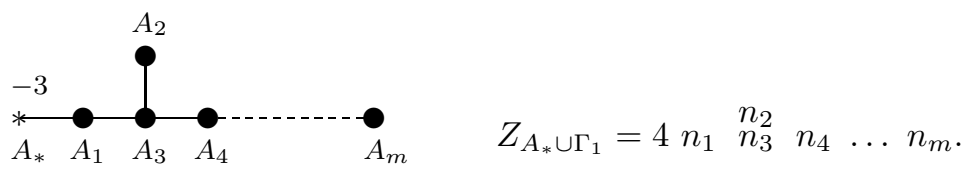

Since $A_{i} \cdot Z=A_{i} \cdot(-K)=A_{i}^{2}+2=0,1 \leq i \leq m$, we have the following system of equations:

$$
\begin{aligned}
& \quad-2 n_{1}+4+n_{3}=0 \\
& \left\{\begin{array}{l}
-2 n_{2}+n_{3}=0 \\
-2 n_{3}+n_{1}+n_{2}+n_{4}=0 \\
-2 n_{4}+n_{3}+n_{5}=0 \\
\vdots \\
-2 n_{m-1}+n_{m-2}+n_{m}=0 \\
-2 n_{m}+n_{m-1}=0
\end{array}\right.
\end{aligned}
$$

(6.11) implies

$$
n_{1}=\frac{m}{2} n_{m}, n_{2}=\frac{m-2}{2} n_{m}, n_{j}=(m-j+1) n_{m}, 3 \leq j \leq m .
$$

(6.10) and (6.12) imply $n_{m}=2$ and hence $n_{1}=m$. Recall that

$$
\begin{aligned}
& -1=A_{*} \cdot(-K)=A_{*} \cdot Z \geq 4(-3)+n_{1}=-12+m \\
\Rightarrow \quad & m \leq 11 .
\end{aligned}
$$

Since $4 \leq m \leq 11$, we are in case (1)-case (8) of the proposition.

We next consider $A_{*}$ attaching on $\Gamma_{1}$ in the following form:

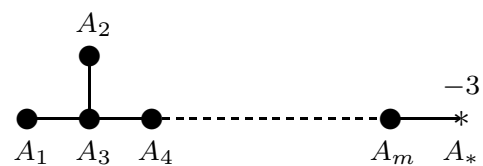

$$
\left.Z\right|_{A_{*} \cup \Gamma_{1}}=n_{1} \quad n_{3} n_{4} n_{4} \ldots n_{m} 4
$$

Since $A_{i} \cdot Z=0,1 \leq i \leq m$, we have the following system of equations:

$$
\left\{\begin{array}{l}
-2 n_{1}+n_{3}=0 \\
-2 n_{2}+n_{3}=0 \\
-2 n_{3}+n_{1}+n_{2}+n_{4}=0 \\
-2 n_{4}+n_{3}+n_{5}=0 \\
\quad \vdots \\
-2 n_{m-1}+n_{m-2}+n_{m}=0 \\
-2 n_{m}+n_{m-1}+4=0 \\
-2 n_{m}+n_{m-1}+4=0
\end{array}\right.
$$

(6.13) implies $n_{3}=n_{4}=\cdots=n_{m}=2 n_{1}=2 n_{2}$. By (6.14), we know that $n_{1}=2$. Therefore we are in case (9) of the proposition. 
Proposition 6.11. Let $\Gamma$ be the minimal resolution graph of a minimally elliptic singularity with fundamental cycle $Z$. Let $\Gamma^{\prime}$ be the subgraph of $\Gamma$ obtained by removing all the effective components of $\Gamma$. Let $A_{*}$ be an effective component of $\Gamma$. Suppose that $\Gamma_{1}$ is a connected component of $\Gamma^{\prime}$ which corresponds to either the $E_{6}, E_{7}$ or $E_{8}$ graph in case (3)-case (5) of Theorem 4.2 . Suppose also that $\Gamma_{1}$ intersects with $A_{*}$ but is disjoint from other effective components. Let $Z_{1}$ be the fundamental cycle of $\Gamma_{1}$. Suppose $A_{*} \cdot Z_{1}=1$. If the coefficient $z_{*}$ of $A_{*}$ in $Z$ is four and $A_{*}^{2} \leq-3$, then $A_{*} \cup \Gamma_{1}$ and the restriction of $Z$ on $A_{*} \cup \Gamma_{1}$ must be of the form

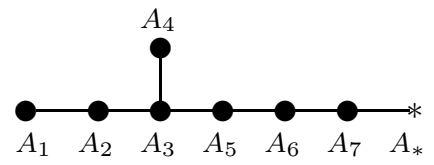

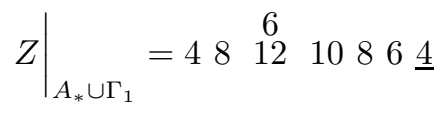

Proof. By Theorem 4.2. $A_{*}$ attaching on $E_{6}$ must be of the following form:

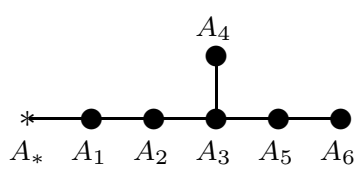

$$
\left.Z\right|_{A_{*} \cup \Gamma_{1}}=4 n_{1} n_{2} \quad n_{3} n_{5} n_{6}
$$

Since $A_{i} \cdot Z=A_{i}(-K)=0$ for $1 \leq i \leq 6$, we have the following system of equations:

$$
\left\{\begin{array}{l}
-2 n_{1}+4+n_{2}=0 \\
-2 n_{2}+n_{1}+n_{3}=0 \\
-2 n_{3}+n_{2}+n_{4}+n_{5}=0 \\
-2 n_{4}+n_{3}=0 \\
-2 n_{5}+n_{3}+n_{6}=0 \\
-2 n_{6}+n_{5}=0,
\end{array}\right.
$$

which imply $n_{6}=\frac{16}{3}$. This contradicts the fact that $n_{6}$ is an integer.

By Theorem 4.2, $A_{*}$ attaching on $E_{7}$ must be of the following form:

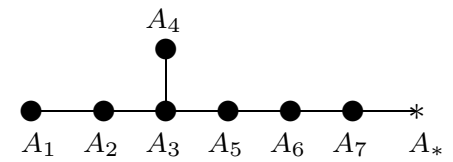

$$
\left.Z\right|_{A_{*} \cup \Gamma_{1}}=n_{1} n_{2} \quad n_{3} n_{3} n_{5} n_{6} n_{7} 4
$$

Since $A_{i} \cdot Z=A_{i} \cdot(-K)=0$ for $1 \leq i \leq 7$, we have the following system of equations:

$$
\left\{\begin{array}{l}
-2 n_{1}+n_{2}=0 \\
-2 n_{2}+n_{1}+n_{3}=0 \\
-2 n_{3}+n_{2}+n_{4}+n_{5}=0 \\
-2 n_{4}+n_{3}=0 \\
-2 n_{5}+n_{3}+n_{6}=0 \\
-2 n_{6}+n_{5}+n_{7}=0 \\
-2 n_{7}+n_{6}+4=0 .
\end{array}\right.
$$

An easy exercise shows that we are in the form of the proposition.

By Theorem 4.2, $A_{*}$ cannot attach on $E_{8}$ because $A_{*} \cdot Z_{1} \geq 2$. 
Theorem 6.12. Let $(V, p)$ be a germ of minimally elliptic singularity. Let $\pi$ : $M \longrightarrow V$ be the minimal resolution of $p$. If case (1) of Proposition 6.2 holds, i.e., there exists only one effective component $A_{*}$, and $A_{*}^{2}=-3, z_{*}=4$, then the weighted dual graph $\Gamma$ of the exceptional set is one of the following forms.

(1)

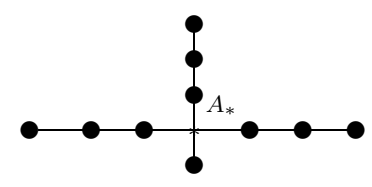

1

2

3

$Z=12 \quad 3 \quad \underline{4} \quad 3 \quad 2 \quad 1$

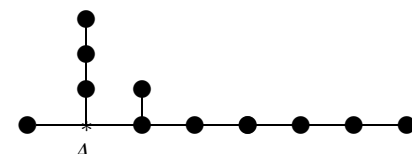

1

2

$3 \quad 3$

$(2)$

$A_{*}$

$Z=2 \quad \underline{4} \quad 6 \quad 5 \quad 4 \quad 321$

(3)

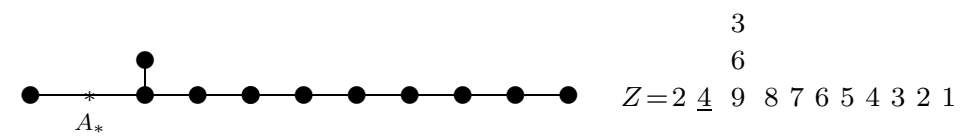

(4)

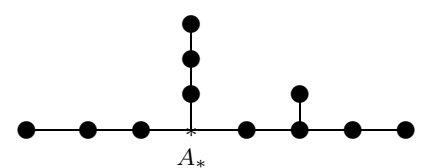

1

2

33

$Z=123 \quad \underline{4} \quad 5 \quad 6 \quad 42$

(5)

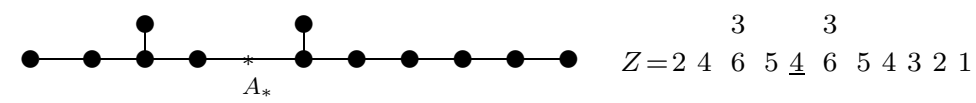

Proof. Let $\Gamma^{\prime}$ be the graph obtained by deleting $A_{*}$ from $\Gamma$. Let $\Gamma_{1}, \ldots, \Gamma_{m}$ be the connected components of $\Gamma^{\prime}$ with fundamental cycles $Z_{1}, \ldots, Z_{m}$ respectively. Since $z_{*}=4$, in view of Proposition 6.3, we have $A_{*} \cdot Z_{i}=1$ for $1 \leq i \leq m$. By Proposition 6.9, Proposition 6.10 and Proposition 6.11, we have

$$
\left\{A_{*} \cdot Z / \Gamma_{1}, \ldots, A_{*} \cdot Z / \Gamma_{m}\right\} \subseteq\{2,3,4,5,6,7,8,9,10,11\}
$$

Since the singularity is minimally elliptic, we have

$$
A_{*} \cdot\left(Z-4 A_{*}\right)=-A_{*} \cdot\left(K+4 A_{*}\right)=A_{*}^{2}+2-4 A_{*}^{2}=11 .
$$


Observe that we can write

$$
\begin{aligned}
11 & =2+2+2+2+3 \\
& =2+2+2+5 \\
& =2+2+3+4 \\
& =2+2+7 \\
& =2+3+3+3 \\
& =2+3+6 \\
& =2+4+5 \\
& =2+9 \\
& =3+3+5 \\
& =3+4+4 \\
& =3+8 \\
& =4+7 \\
& =5+6 \\
& =11 .
\end{aligned}
$$

In case of $11=2+2+2+2+3$, by Proposition 6.9 (1) and (2) we obtain a graph with the proposed fundamental cycle

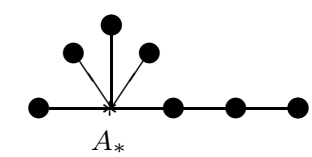

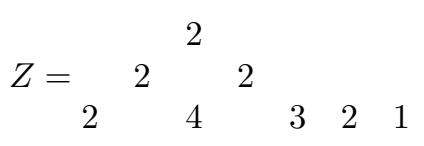

On the other hand one may find a positive cycle on the graph

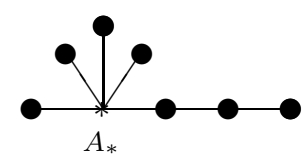

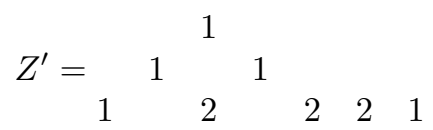

with $Z^{\prime}<Z$ that also satisfies Definition 2.1. Therefore the proposed fundamental cycle $Z$ does not satisfy the minimum condition stated in Definition 2.1. Hence there is no dual graph produced from this case.

Similarly, by Propositions 6.9, 6.10 and 6.11 together with Definition 2.1, in cases $11=2+2+2+5,11=2+2+3+4$ and $11=2+2+7$, there is no dual graph produced. In the case of $11=1+3+3+3$, we have case (1). In the case of $11=2+3+6$, we only have case (2). In the case of $11=2+4+5$, there is no dual graph produced. In the case of $11=2+9$, we only have case $(3)$. In the case of $11=3+3+5$, we have case (4). In the cases $11=3+4+4,11=3+8$ and $11=4+7$, there is no dual graph produced. In the case of $11=5+6$, we only have case (5). Finally case $11=11$ does not produce any dual graph.

Proposition 6.13. Let $\Gamma$ be the minimal resolution graph of a minimally elliptic singularity with fundamental cycle $Z$. Let $\Gamma^{\prime}$ be the subgraph of $\Gamma$ obtained by removing all the effective components of $\Gamma$. Let $A_{*}$ be an effective component of $\Gamma$. Suppose that $\Gamma_{1}$ is a connected component of $\Gamma^{\prime}$ which corresponds to a rational 
double point graph in Theorem 4.2. Suppose also that $\Gamma_{1}$ intersects with $A_{*}$ but is disjoint from other effective components. Let $Z_{1}$ be the fundamental cycle on $\Gamma_{1}$. Suppose $A_{*} \cdot Z_{1}=1$. If the coefficient $z_{*}$ of $A_{*}$ in $Z$ is 2 and $A_{*}^{2}=-4$, then $A_{*} \cup \Gamma_{1}$ and the restriction of $Z$ on $A_{*} \cup \Gamma_{1}$ must be one of the following forms.

(1)

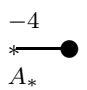

$(2)$

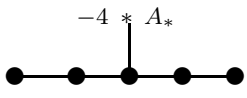

(3)

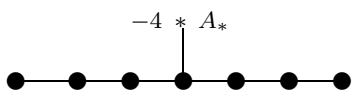

(4)

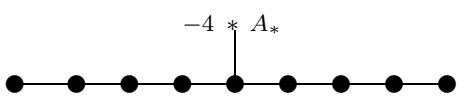

(5)

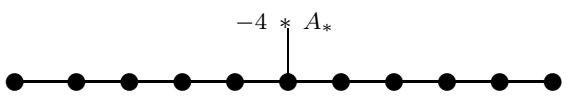

(6)

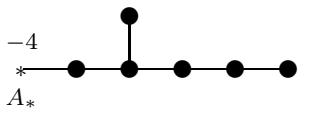

(7)

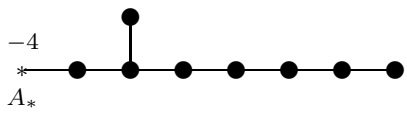

(8)

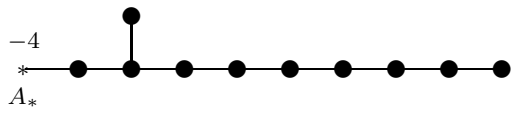

(9)

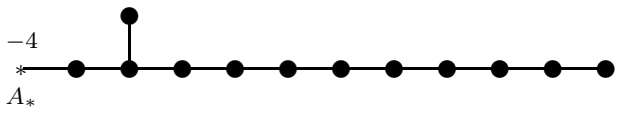

$(10)$

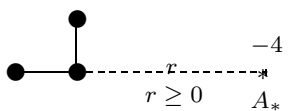

(11)

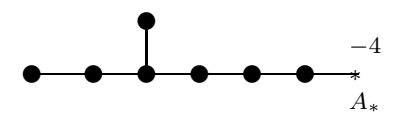

$\left.Z\right|_{A_{*} \cup \Gamma_{1}}=\underline{2} 1$

$\left.Z\right|_{A_{*} \cup \Gamma_{1}}=1 \quad \begin{array}{llll}2 & \frac{2}{3} & 2 & 1\end{array}$

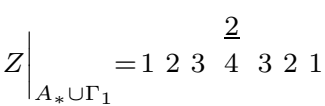

$\left.Z\right|_{A_{*} \cup \Gamma_{1}}=1234 \frac{2}{5} 4321$

$\left.Z\right|_{A_{*} \cup \Gamma_{1}}=12345 \stackrel{2}{6} 54321$

$\left.Z\right|_{A_{*} \cup \Gamma_{1}}=\underline{2} \begin{array}{ccccc}2 & 4 & 3 & 2 & 1\end{array}$

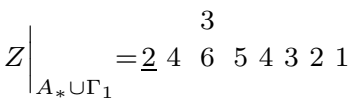

$\left.Z\right|_{A_{*} \cup \Gamma_{1}}=\underline{2} 5 \quad \begin{array}{r}4 \\ 8\end{array}$

$\left.Z\right|_{A_{*} \cup \Gamma_{1}}=\underline{2} 6 \quad 10987654321$

$\left.Z\right|_{A_{*} \cup \Gamma_{1}}=1 \quad 2 \underbrace{2 \ldots \ldots 2}_{r \geq 0} \underline{2}$

$\left.Z\right|_{A_{*} \cup \Gamma_{1}}=24 \begin{array}{lllll}3 & 6 & 5 & 4 & 3\end{array}$

Proof. The proof is similar to those of Propositions 6.9, 6.10 and 6.11,

Theorem 6.14. Let $(V, p)$ be a germ of minimally elliptic singularity. Let $\pi$ : $M \rightarrow V$ be the minimal resolution of $p$. If case (2) of Proposition 6.2 holds, i.e., if there exists only one effective component $A_{*}$, and $A_{*}^{2}=-4, z_{*}=2$, then the weighted dual graph $\Gamma$ of the exceptional set is one of the following forms. 
(1)

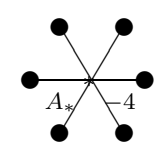

(2)

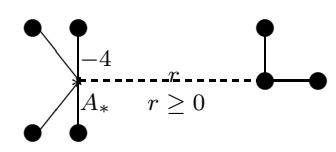

(3)

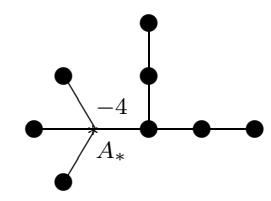

(4)

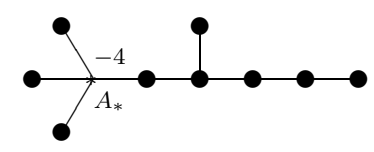

(5)

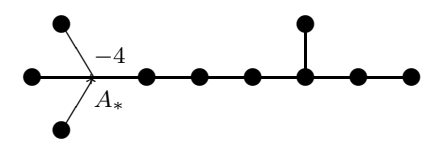

(6)

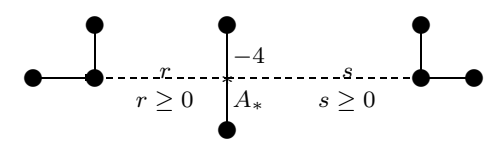

(7)

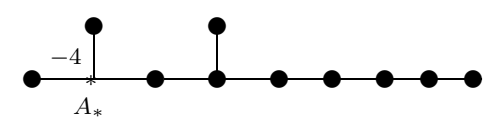

(8)

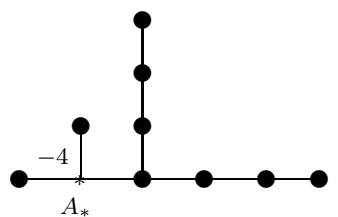

(9)

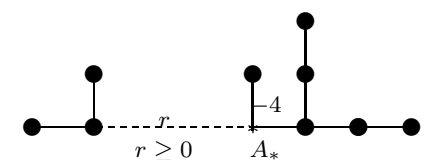

(10)

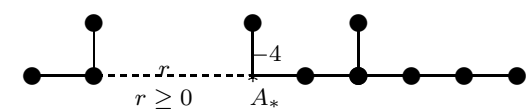

$$
\begin{aligned}
& Z=1{ } \stackrel{1}{ } \underline{2}_{1}{ }^{1} 1 \\
& Z=\begin{array}{rrrrr}
1 & 1 & & 1 \\
& 2 & \ldots & 2 & 1 \\
1 & 1 & &
\end{array}
\end{aligned}
$$

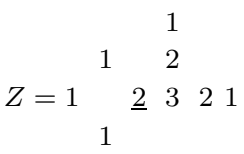

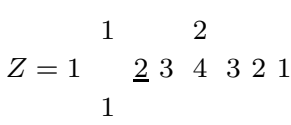

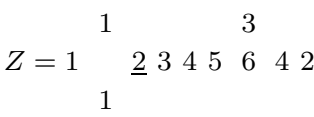$$
\begin{array}{lllllll} 
& 1 & & 1 & & 1 \\
& & & & & & \\
1 & 2 & \ldots & \ldots & 2 & 2 & 1 \\
1 & & & &
\end{array}
$$$$
Z=1 \quad \begin{array}{rrrrrrrr} 
& 1 & 3 & & & & \\
Z & 4 & 6 & 5 & 4 & 3 & 2 & 1
\end{array}
$$

$$
\begin{aligned}
& \begin{array}{r}
1 \\
2
\end{array} \\
& Z=1 \underline{2} 4 \quad 3 \quad 21
\end{aligned}
$$

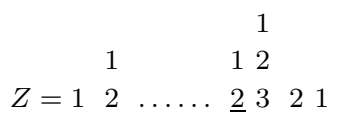

$Z=1 \quad \begin{array}{rrrrrrrrrr} & 1 & & & 1 & & 2 & & & \\ Z & 2 & \ldots & \underline{2} & 3 & 4 & 3 & 2 & 1\end{array}$ 
(11)

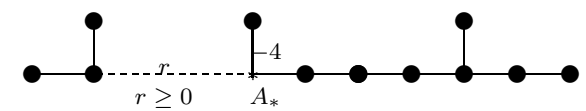

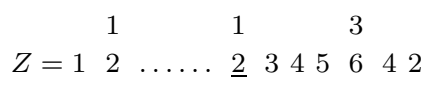

(12)

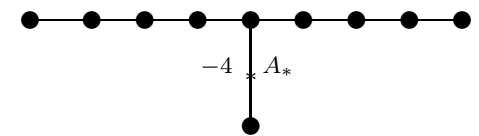

$$
Z=123454321
$$$$
\frac{2}{1}
$$

(13)

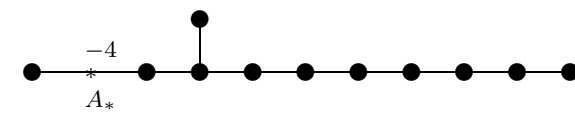

$$
Z=1 \underline{2} 5 \quad \begin{aligned}
& 4 \\
& 8
\end{aligned} 76654321
$$

(14)
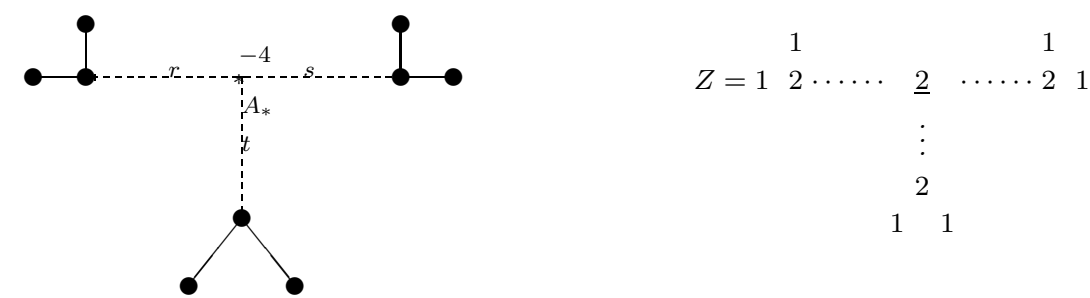

(15)
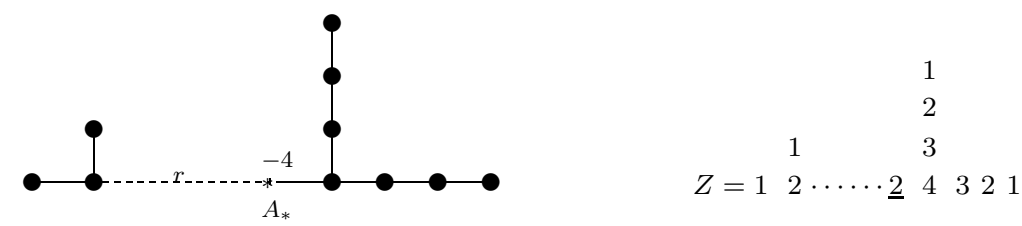

(16)

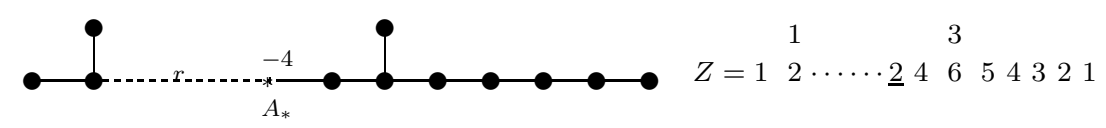

(17)

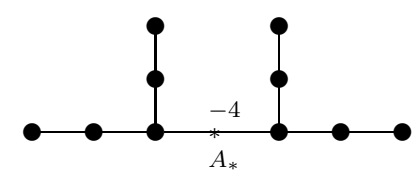

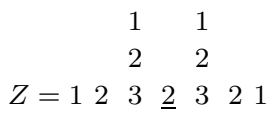

(18)

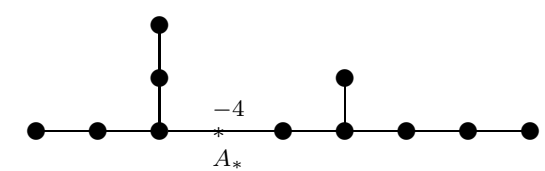

$$
\begin{aligned}
& \begin{array}{ll}
1 & \\
2 &
\end{array} \\
& Z=12 \begin{array}{lllllll}
2 & 3 & 3 & 4 & 3 & 2 & 1
\end{array}
\end{aligned}
$$


(19)

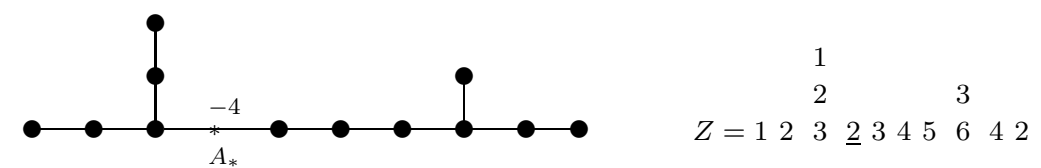

(20)

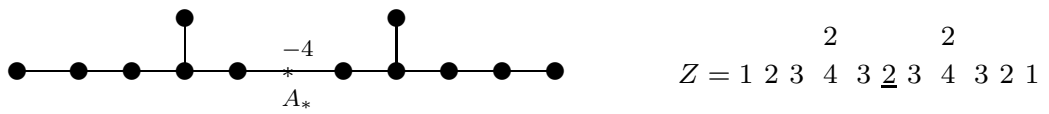

(21)

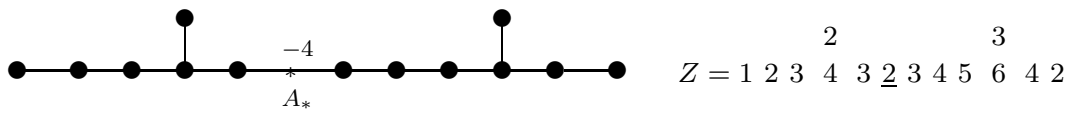

(22)

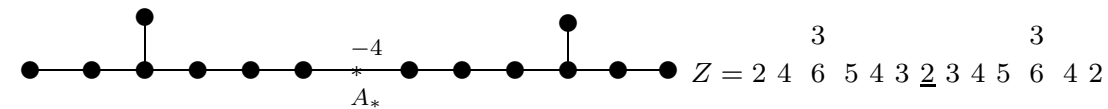

(23)

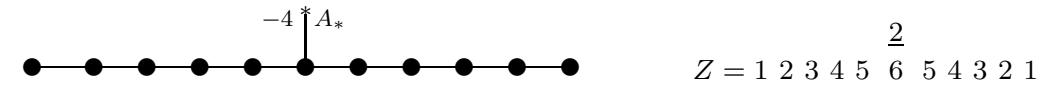

(24)

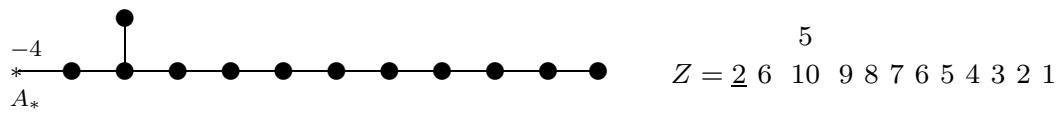

Proof. The proof is the same as those given in Theorem6.12, By Proposition 6.13. we have

$$
\left\{A_{*} \cdot Z / \Gamma_{m}, \ldots, Z_{*} \cdot Z / \Gamma_{m}\right\} \subseteq\{1,2,3,4,5,6\} .
$$

Since the singularity is minimally elliptic, we have

$$
A_{*} \cdot\left(Z-2 A_{*}\right)=-A_{*}\left(K+2 A_{*}\right)=-A_{*}^{2}+2=6 .
$$

Observe that we can write

$$
\begin{aligned}
& 6=1+1+1+1+1+1 \quad(\text { case }(1)) \\
& =1+1+1+1+2 \quad(\text { case }(2)) \\
& =1+1+1+3 \quad \text { (case }(3) \text {, case }(4) \text {, case }(5)) \\
& =1+1+2+2 \quad(\text { case }(6)) \\
& =1+1+4 \quad(\text { case }(7), \text { case }(8)) \\
& =1+2+3 \quad \text { (case }(9) \text {, case }(10) \text {, case }(11)) \\
& =1+5 \quad(\text { case }(12), \text { case }(13)) \\
& =2+2+2 \quad(\text { case }(14)) \\
& =2+4 \quad(\text { case }(15), \text { case }(16))
\end{aligned}
$$




$$
\begin{array}{ll}
=3+3 & (\text { case }(17)-\text { case }(22)) \\
=6 & (\text { case }(23), \text { case }(24)) .
\end{array}
$$

Proposition 6.15. Let $\Gamma$ be the minimal resolution graph of a minimally elliptic singularity with fundamental cycle $Z$. Let $\Gamma^{\prime}$ be the subgraph of $\Gamma$ obtained by removing all the effective components of $\Gamma$. Let $A_{*}$ be an effective component of $\Gamma$. Suppose that $\Gamma_{1}$ is a connected component of $\Gamma^{\prime}$ which corresponds to a rational double point graph in Theorem 4.2. Suppose also that $\Gamma_{1}$ intersects with $A_{*}$ but is disjoint from other effective components. Let $Z_{1}$ be the fundamental cycle on $\Gamma_{1}$. Suppose $A_{*} \cdot Z_{1}=1$. If the coefficient $z_{*}$ of $A_{*}$ in $Z$ is 1 and $A_{*}^{2}=-6$, then such a graph does not exist.

Proof. The proof is similar to those of Propositions 6.9, 6.10 and 6.11.

Theorem 6.16. Let $(V, p)$ be a germ of minimally elliptic singularity. Let $\pi: M \rightarrow$ $V$ be the minimal resolution of p. If case (3) of Proposition 6.2 holds, i.e., if there exists one effective component $A_{*}$, and $A_{*}^{2}=-6, z_{*}=1$, then the weighted dual graph $\Gamma$ of the exceptional set is one of the following forms.

(1)

(2) (i)

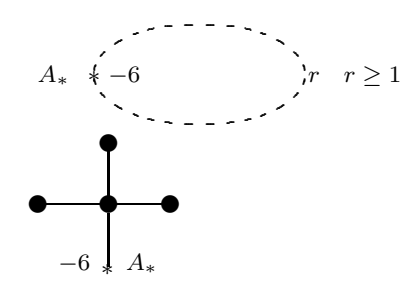

(ii)

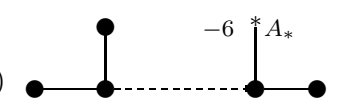

(3)

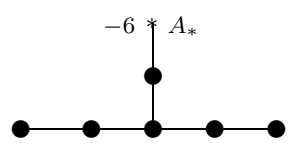

(4)

(5)
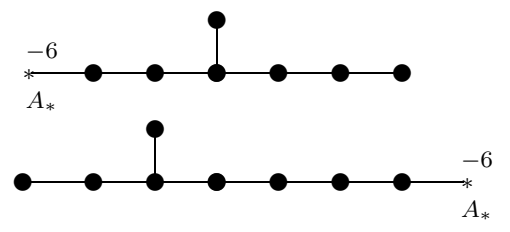

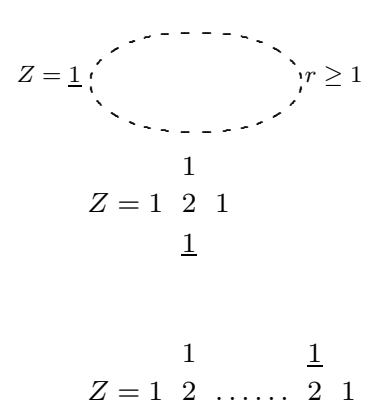

$\underline{1}$

$Z=12321$

2

3

$Z=24 \quad 6 \quad 5432 \underline{1}$

Proof. This follows easily from Proposition 6.3, Corollary 6.4 and Proposition 6.15,

Proposition 6.17. Let $\Gamma$ be the minimal resolution graph of a minimally elliptic singularity with fundamental cycle $Z$. Let $\Gamma^{\prime}$ be the subgraph of $\Gamma$ obtained by removing all the effective components of $\Gamma$. Let $A_{*}$ be an effective component of $\Gamma$. Suppose that $\Gamma_{1}$ is a connected component of $\Gamma^{\prime}$ which corresponds to a rational 
double point graph in Theorem 4.2. Suppose also that $\Gamma_{1}$ intersects with $A_{*}$ but is disjoint from other effective components. Let $Z_{1}$ be the fundamental cycle on $\Gamma_{1}$. Suppose $A_{*} \cdot Z_{1}=1$. If the coefficient $z_{*}$ of $Z_{*}$ in $Z$ is 2 and $A_{*}^{2}=-3$, then $A_{*} \cup \Gamma_{1}$ and the restriction of $Z$ on $A_{*} \cup \Gamma_{1}$ must be one of the following forms.

(1)

$(2)$

(3)

(4)

(5)

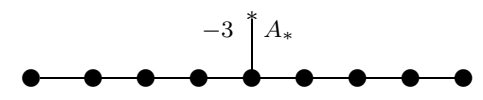

(6)

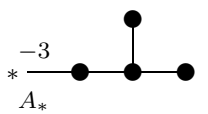

(7)

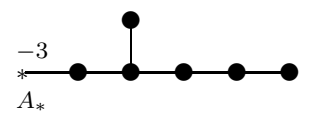

(8)

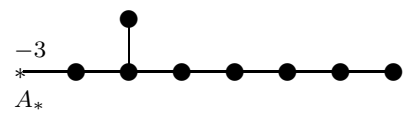

(9)

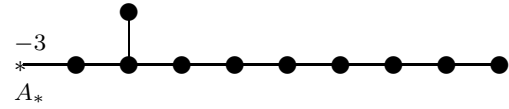

(10)

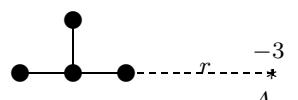

(11)

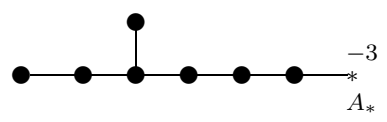

$\left.Z\right|_{A_{*} \cup \Gamma_{1}}=\underline{2} 1$

$\left.Z\right|_{A_{*} \cup \Gamma_{1}}=1 \stackrel{\underline{2}}{2} 1$

$\left.Z\right|_{A_{*} \cup \Gamma_{1}}=12 \begin{array}{llll} & 2 & 3 & 2\end{array}$

$\left.Z\right|_{A_{*} \cup \Gamma_{1}}=123 \quad \begin{array}{lllll}4 & 3 & 2 & \end{array}$

$\left.Z\right|_{A_{*} \cup \Gamma_{1}}=1234 \begin{aligned} & \underline{2} \\ & 5\end{aligned} 4321$

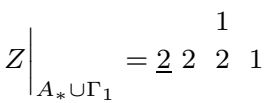

$\left.Z\right|_{A_{*} \cup \Gamma_{1}}=\underline{2} 3 \quad 4 \quad 321$

$\left.Z\right|_{A_{*} \cup \Gamma_{1}}=\underline{2} 4 \quad 6 \quad 54321$

$\left.Z\right|_{A_{*} \cup \Gamma_{1}}=\underline{2} 5 \quad 8 \quad 7 \quad 7654321$

$\left.Z\right|_{A_{*} \cup \Gamma_{1}}=1 \quad \begin{array}{rrrr}1 & & \\ & 2 & 2 & \ldots\end{array}$

$\left.Z\right|_{A_{*} \cup \Gamma_{1}}=24 \begin{array}{lllll}3 & 5 & 43 & \underline{2}\end{array}$

Proof. The proof is similar to those of Propositions 6.9, 6.10 and 6.11.

Proposition 6.18. Let $\Gamma$ be the minimal resolution graph of a minimally elliptic singularity with fundamental cycle $Z$. Let $\Gamma^{\prime}$ be the subgraph of $\Gamma$ obtained by removing all the effective components of $\Gamma$. Let $A_{* 1}$ and $A_{* 2}$ be two effective components of $\Gamma$. Suppose that $\Gamma_{1}$ is a connected component of $\Gamma^{\prime}$ which corresponds to a rational double point graph in Theorem 4.2, Suppose also that $\Gamma_{1}$ intersects with both $A_{* 1}$ and $A_{* 2}$, but no other effective component. Let $Z_{1}$ be the fundamental cycle on $\Gamma_{1}$. Suppose $A_{* 1} \cdot Z_{1}=A_{* 2} \cdot Z_{1}=1$. If $A_{* 1} \cdot A_{* 2}=0$ and the coefficients 
$z_{* 1}$ of $A_{* 1}$ and $z_{* 2}$ of $A_{* 2}$ in $Z$ are 2 and $A_{* 1}^{2}=A_{* 2}^{2}=-3$, then $A_{* 1} \cup A_{* 2} \cup \Gamma_{1}$ and the restriction of $Z$ on $A_{* 1} \cup A_{* 2} \cup \Gamma_{1}$ must be one of the following forms.

(1)

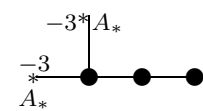

(2)

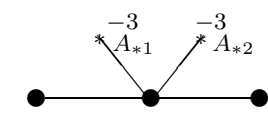

(3)

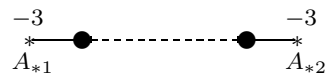

(4)

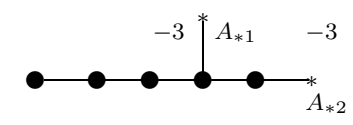

(5)

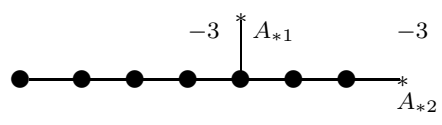

(6)

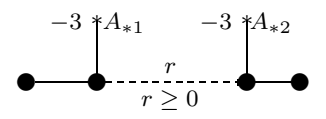

(7)

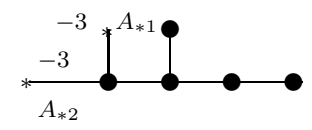

(8)

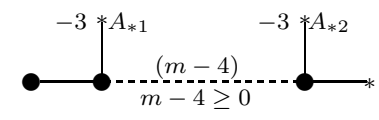

(9)

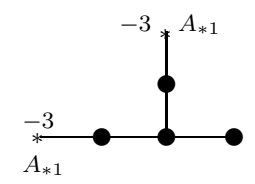

(10)

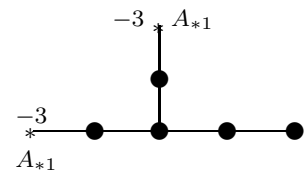

$\left.Z\right|_{A_{* 1} \cup A_{* 2} \cup \Gamma_{1}}=\underline{2} \frac{2}{3} 21$

$\left.Z\right|_{A_{* 1} \cup A_{* 2} \cup \Gamma_{1}}=2^{\underline{2}}{ }^{\underline{2}} 2$

$\left.Z\right|_{A_{* 1} \cup A_{* 2} \cup \Gamma_{1}}=\underline{2} \underbrace{2 \ldots \ldots 2}_{r \geq 1} \underline{2}$

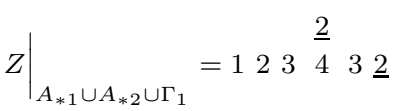

$\left.Z\right|_{A_{* 1} \cup A_{* 2} \cup \Gamma_{1}}=12234 \overline{5} 44 \underline{2}$

$\left.Z\right|_{A_{* 1} \cup A_{* 2} \cup \Gamma_{1}}=2 \stackrel{\underline{2}}{4} \underbrace{4 \ldots \ldots 4}_{r \geq 0} \stackrel{\underline{2}}{4} 2$

$\left.Z\right|_{A_{* 1} \cup A_{* 2} \cup \Gamma_{1}}=\underline{2} \begin{aligned} & \underline{2} 3 \\ & 5\end{aligned}$

$\left.Z\right|_{A_{* 1} \cup A_{* 2} \cup \Gamma_{1}}=2 \stackrel{\frac{2}{4}}{\underbrace{4 \ldots \ldots 4}_{m-4 \geq 0}} \stackrel{\underline{2}}{4} \underline{2}$

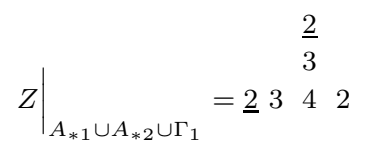

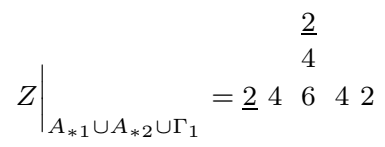


(11)

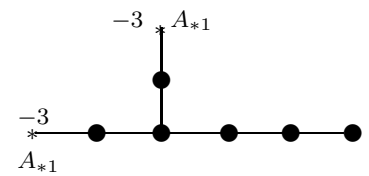

(12)

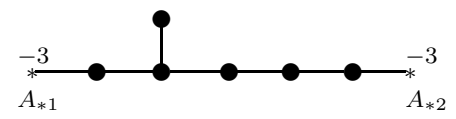

(13)

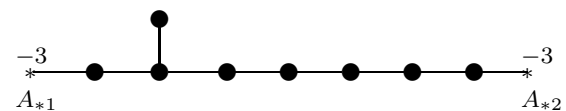

(14)

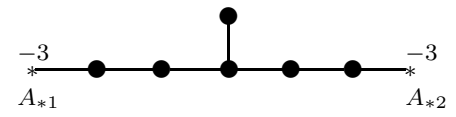

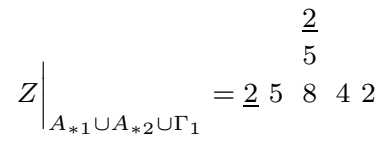

$\left.Z\right|_{A_{* 1} \cup A_{* 2} \cup \Gamma_{1}}=\underline{2} 4 \quad 6 \quad 543 \underline{2}$

$\left.Z\right|_{A_{* 1} \cup A_{* 2} \cup \Gamma_{1}}=\underline{2} 5 \quad \begin{array}{r}4 \\ 5\end{array}$

$\left.Z\right|_{A_{* 1} \cup A_{* 2} \cup \Gamma_{1}}=\underline{2} 46 \quad 8 \quad 8 \quad 64 \underline{2}$

Proof. (I) Assume that $\Gamma_{1}$ is of the form of case (1) in Theorem 4.2.

Consider $A_{* 1}$ and $A_{* 2}$ attaching on $\Gamma_{1}$ in the following form:

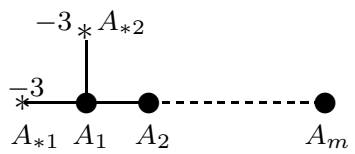

$$
\left.Z\right|_{A_{* 1} \cup A_{* 2} \cup \Gamma_{1}}=2 \begin{array}{cccc}
2 & n_{1} & n_{2} & \ldots
\end{array} n_{m} .
$$

As in the proof of Proposition 6.9, we have $m=1$ or $m=3$. If $m=1$, then we are in case (3). If $m=3$, then we are in case (1).

Consider $A_{* 1}$ and $A_{* 2}$ attaching on $\Gamma_{1}$ in the following form:

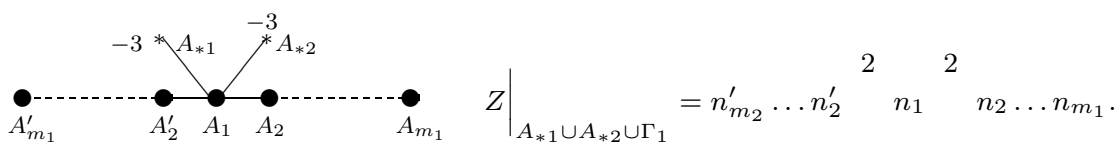

As in the proof of Proposition 6.9, we have either $n_{m_{1}}=3, n_{m_{2}}^{\prime}=1$ or $n_{m_{1}}=$ $2=n_{m_{2}}^{\prime}$.

If $n_{m_{1}}=3, n_{m_{2}}^{\prime}=1$, then $m_{2}=3 m_{1}$ and $n_{1}=3 m_{1}$. Since $-1=A_{* 1}^{2}+2=$ $A_{* 1} \cdot(-K)=A_{* 1} \cdot Z \geq 2(-3)+n_{1} \Rightarrow n_{1}=3 m_{1} \leq 5$, therefore $m_{1}=1, m_{2}=3$ and we are in case (1).

If $n_{m_{1}}=2=n_{m_{2}}^{\prime}$, then $m_{1}=m_{2}$ and $n_{1}=2 m_{1}$. The same argument as above shows that $2 m_{1} \leq 5$, i.e., $m_{1} \leq 2$. If $m_{1}=1$, then we are in case (3). If $m_{1}=2$, then we are in case (2).

Consider $A_{* 1}$ and $A_{* 2}$ attaching a $\Gamma_{1}$ in the following form:

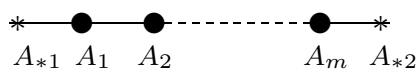

$$
\left.Z\right|_{A_{* 1} \cup A_{* 2} \cup \Gamma_{1}}=2 n_{1} n_{2} \ldots n_{m} 2 .
$$


Since $A_{i} \cdot Z=A_{i} \cdot(-K)=A_{i}^{2}+2=0,1 \leq i \leq m$, we have

$$
\left\{\begin{array}{c}
-2 n_{1}+2+n_{2}=0 \\
-2 n_{2}+n_{1}+n_{3}=0 \\
\vdots \\
-2 n_{m-1}+n_{m-2}+n_{m}=0 \\
-2 n_{m}+n_{m-1}+2=0 .
\end{array}\right.
$$

(6.15) implies

$$
n_{j}=j n_{1}-2(j-1), \quad 2 \leq j \leq m .
$$

(6.16) and (6.17) imply $n_{1}=2=n_{2}=\cdots=n_{m}$. We are in case (3).

Consider $A_{* 1}$ and $A_{* 2}$ attaching on $\Gamma_{1}$ in the following form:

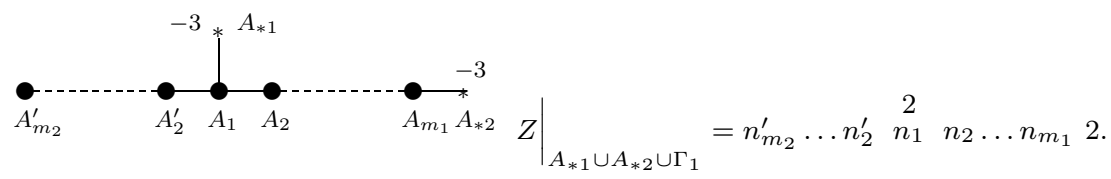

Since $A_{i} \cdot Z=A_{i} \cdot(-K)=A_{i}^{2}+2=0,1 \leq i \leq m$ and $A_{j}^{\prime} \cdot Z=A_{j}^{\prime} \cdot(-K)=$ $A_{j}^{\prime 2}+2=0,2 \leq j \leq m_{2}$, we have

$$
\begin{aligned}
& \left\{\begin{array}{c}
-2 n_{m_{1}}+n_{m_{1}-1}+2=0 \\
-2 n_{m_{1}-1}+n_{m_{1}-2}+n_{m_{1}}=0 \\
\vdots \\
-2 n_{3}+n_{2}+n_{4}=0 \\
-2 n_{2}+n_{1}+n_{3}=0,
\end{array}\right. \\
& \left\{\begin{array}{c}
-2 n_{m_{2}}^{\prime}+n_{m_{2}-1}^{\prime}=0 \\
-2 n_{m_{2}-1}^{\prime}+n_{m_{2}-2}^{\prime}+n_{m_{2}}^{\prime}=0 \\
\vdots \\
-2 n_{3}^{\prime}+n_{2}^{\prime}+n_{4}^{\prime}=0 \\
-2 n_{2}^{\prime}+n_{1}+n_{3}^{\prime}=0, \\
2-2 n_{1}+n_{2}+n_{2}^{\prime}=0 .
\end{array}\right.
\end{aligned}
$$

(6.18) implies

$$
n_{j}=\left(m_{1}-j+1\right) n_{m_{1}}-2\left(m_{1}-j\right), \quad 1 \leq j \leq m_{1}-1 .
$$

(6.19) implies

$$
n_{j}^{\prime}=\left(m_{2}-j+1\right) n_{m_{2}}^{\prime}, \quad 1 \leq j \leq m_{2}-1 .
$$

(6.21) and (6.22) imply

$$
m_{1} n_{m_{1}}-2\left(m_{1}-1\right)=m_{2} n_{m_{2}}^{\prime}=n_{1} .
$$

(6.20), (6.21) and (6.22) imply $n_{m_{1}}+n_{m_{2}}^{\prime}=4$. We have either $n_{m_{1}}=3, n_{m_{2}}^{\prime}=1$ or $n_{m_{1}}=2=n_{m_{2}}^{\prime}$.

If $n_{m_{1}}=3$ and $n_{m_{2}}^{\prime}=1$, then (6.23) implies $m_{2}=m_{1}+2=n_{1} .-1=A_{* 1}^{2}+2=$ $A_{* 1} \cdot(-K)=A_{* 1} \cdot Z \geq 2(-3)+n_{1}$ implies $m_{1}+2=n_{1} \leq 5$, i.e., $m_{1} \leq 3$. If 
$m_{1}=1$, then $m_{2}=3$ and we are in case (1). If $m_{1}=2$, then $m_{2}=4$ and we are in case (4). If $m_{1}=3$, then $m_{2}=5$ and we are in case (5).

If $n_{m_{1}}=2=n_{m_{2}}^{\prime}$, then (6.23) implies $m_{2}=1$ and we are in case (3).

Consider $A_{* 1}$ and $A_{* 2}$ attaching on $\Gamma_{1}$ in the following form:

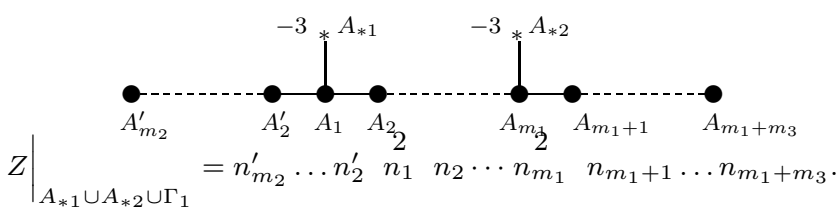

By the same argument as before, we have the following equations:

$$
\begin{aligned}
& \left\{\begin{array}{l}
-2 n_{m_{2}}^{\prime}+n_{m_{2}-1}^{\prime}=0 \\
-2 n_{m_{2}-1}^{\prime}+n_{m_{2}-2}^{\prime}+n_{m_{2}}^{\prime}=0 \\
\vdots \\
-2 n_{3}^{\prime}+n_{2}^{\prime}+n_{4}^{\prime}=0 \\
-2 n_{2}^{\prime}+n_{1}+n_{3}^{\prime}=0,
\end{array}\right. \\
& -2 n_{1}+n_{2}^{\prime}+n_{2}+2=0 \text {, }
\end{aligned}
$$$$
\left\{\begin{array}{l}
-2 n_{2}+n_{1}+n_{3}=0 \\
-2 n_{3}+n_{2}+n_{4}=0 \\
\vdots \\
-2 n_{m_{1}-2}+n_{m_{1}-3}+n_{m_{1}-1}=0 \\
-2 n_{m_{1}-1}+n_{m_{1}-2}+n_{m_{1}}=0,
\end{array}\right.
$$

$$
-2 n_{m_{1}}+n_{m_{1}-1}+n_{m_{1}+1}+2=0,
$$

$$
\left\{\begin{array}{l}
-2 n_{m_{1}+1}+n_{m_{1}}+n_{m_{1}+2}=0 \\
-2 n_{m_{1}+2}+n_{m_{1}+1}+n_{m_{1}+3}=0 \\
\vdots \\
-2 n_{m_{1}+m_{3}-1}+n_{m_{1}+m_{3}-2}+n_{m_{1}+m_{3}}=0 \\
-2 n_{m_{1}+m_{3}}+n_{m_{1}+m_{3}-1}=0 .
\end{array}\right.
$$

(6.24) implies

$$
n_{j}^{\prime}=\left(m_{2}-j+1\right) n_{m_{2}}^{\prime}, \quad 1 \leq j \leq m_{2}-1 .
$$

(6.25) and (6.29) imply

$$
n_{2}=\left(m_{2}+1\right) n_{m_{2}}^{\prime}-2 \text {. }
$$

(6.30) and (6.26) imply

$$
n_{j}=\left(m_{2}+j-1\right) n_{m_{2}}^{\prime}-2(j-1), \quad 3 \leq j \leq m_{1} .
$$

(6.28) implies

$$
n_{m_{1}+j}=\left(m_{3}-j+1\right) n_{m_{1}+m_{3}}, \quad 0 \leq j \leq m_{3}-1 .
$$

(6.31) and (6.32) imply

$$
n_{m_{1}}=\left(m_{2}+m_{1}-1\right) n_{m_{2}}^{\prime}-2\left(m_{1}-1\right)=\left(m_{3}+1\right) n_{m_{1}+m 2} .
$$


(6.31), (6.32) and (6.27) imply

$$
\left(m_{2}+m_{1}\right) n_{m_{2}}^{\prime}-2 m_{1}=m_{3} n_{m_{1}+m_{3}}+2 .
$$

(6.33) and (6.34) imply $n_{m_{2}}^{\prime}+n_{m_{1}+m_{3}}=4$. Therefore we have either $n_{m_{2}}^{\prime}=1$, $n_{m_{1}+m_{3}}=3$ or $n_{m_{2}}^{\prime}=2=n_{m_{1}+m_{3}}$.

If $n_{m_{2}}^{\prime}=1$ and $n_{m_{1}+m_{3}}=3$, then $m_{2}=m_{1}+3 m_{3}+2$ by $(6.34)$ and $n_{1}=m_{2}$ by (6.29). Since $-1=A_{* 1}^{2}+2=A_{* 1}(-K)=A_{* 1} \cdot Z \geq 2(-3)+n_{1}$, we have $m_{2} \leq 5$. Hence $m_{1}+3 m_{3} \leq 3$. Since $m_{1} \geq 2$, we have either $m_{3}=0, m_{1}=2, m_{2}=4$, or $m_{3}=0, m_{1}=3, m_{2}=5$. If $m_{3}=0, m_{1}=2, m_{2}=4$, then we are in case (4). If $m_{3}=0, m_{1}=3, m_{2}=5$, then we are in case (5).

If $n_{m_{2}}^{\prime}=2=n_{m_{1}+m_{3}}$, then $n_{1}=2 m_{2}$ and $m_{2}=m_{3}+1$ by (6.33). Since $-1=A_{* 1}^{2}+2=A_{* 1} \cdot(-K)=A_{* 1} \cdot Z \geq 2(-3)+n_{1}$, we have $2 m_{2} \leq 5$, which implies $m_{2} \leq 2$ and $m_{3} \leq 1$. If $m_{3}=0$ and $m_{2}=1$, then we are in case (3). If $m_{3}=1$ and $m_{2}=2$, then $n_{j}=4,1 \leq j \leq m_{1}$ and we are in case (6).

(II) Assume that $\Gamma_{1}$ is of the form $D_{m}$ of case (2) in Theorem 4.2

Consider $A_{* 1}$ and $A_{* 2}$ attaching on $\Gamma_{1}$ in the following form:

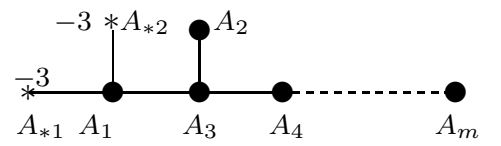

$$
\left.Z\right|_{A_{* 1} \cup A_{* 2} \cup \Gamma_{1}}=2 \begin{array}{ccc}
2 & n_{2} & \\
n_{1} & n_{3} & n_{4} \ldots n_{m} .
\end{array}
$$

As in the proof of Proposition 6.10, we have $n_{m}=2, n_{1}=m, n_{2}=m-2, n_{j}=$ $2(m-j+1), 3 \leq j \leq m$. Since $-1=A_{* 1}^{2}+2=A_{* 1} \cdot(-K)=A_{* 1} \cdot Z \geq 2(-3)+n_{1}$, we have $m \leq 5$. If $m=4$, then we are in case (8). If $m=5$, then we are in case (7).

Consider $A_{* 1}$ and $A_{* 2}$ attaching on $\Gamma_{1}$ in the following form:
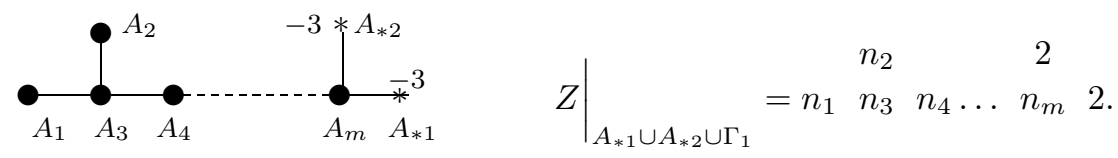

As in the proof of Proposition 6.10, we have $n_{1}=n_{2}=2, n_{3}=\cdots=n_{m}=4$ and we are in case (8).

Consider $A_{* 1}$ and $A_{* 2}$ attaching on $\Gamma_{1}$ in the following form:
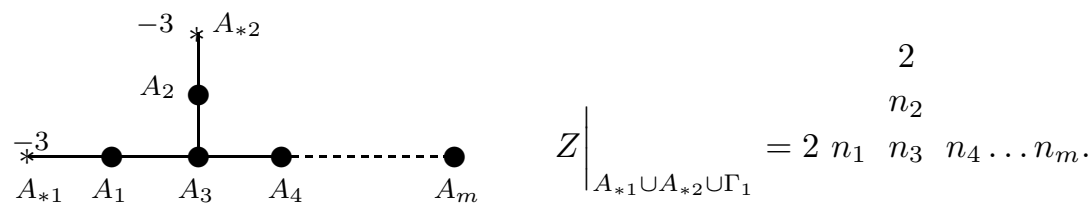

By the same argument as before, we have the following equations:

$$
\begin{aligned}
& \left\{\begin{array}{l}
-2 n_{1}+2+n_{3}=0 \\
-2 n_{2}+2+n_{3}=0 \\
-2 n_{4}+n_{3}+n_{5}=0 \\
\vdots \\
-2 n_{m-1}+n_{m-2}+n_{m}=0 \\
-2 n_{m}+n_{m-1}=0,
\end{array}\right. \\
& -2 n_{3}+n_{1}+n_{2}+n_{4}=0 .
\end{aligned}
$$


(6.35) implies

$$
n_{1}=n_{2}=1+\frac{m-2}{2} n_{m}, \quad n_{j}=(m-j+1) n_{m}, \quad 3 \leq j \leq m .
$$

(6.36) and (6.37) imply $n_{m}=2$ and $n_{1}=n_{2}=m-1$. Since $-1=A_{* 1}^{2}+1=$ $A_{* 1} \cdot(-K)=A_{* 1} \cdot Z \geq 2(-3)+n_{1}$, we have $m \leq 6$. Hence we are in case (9) and case (10) and case (11).

Consider $A_{* 1}$ and $A_{* 2}$ attaching on $\Gamma_{1}$ in the following form:

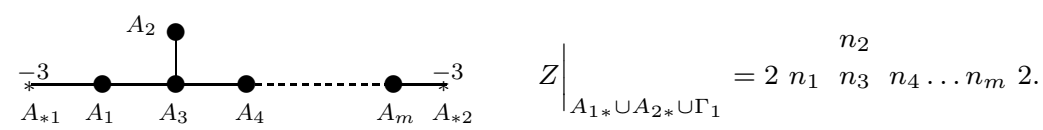

By the same argument as before, we have the following equations:

$$
\begin{aligned}
& \left\{\begin{array}{l}
-2 n_{1}+2+n_{3}=0 \\
-2 n_{2}+n_{3}=0 \\
-2 n_{3}+n_{1}+n_{2}+n_{4}=0 \\
-2 n_{4}+n_{3}+n_{5}=0 \\
\vdots \\
-2 n_{m-1}+n_{m-2}+n_{m}=0
\end{array}\right. \\
& -2 n_{m}+n_{m-1}+2=0
\end{aligned}
$$

(6.38) implies

$$
n_{1}=1+n_{2}, \quad n_{j}=2 n_{2}-(j-3), \quad 3 \leq j \leq m .
$$

(6.39) and (6.40) imply $n_{2}=\frac{m}{2}$. In particular $m$ is even. Since $-1=A_{* 1}^{2}+2=$ $A_{* 1} \cdot(-K)=A_{* 1} \cdot Z \geq 2(-3)+n_{1}$, we have $n_{1} \leq 5$, which implies $1+\frac{m}{2} \leq 5$ and hence $m \leq 8$. If $m=4,6,8$, then we are in case (9), case (12) and case (13) respectively.

(III) Assume that $\Gamma_{1}$ is of the form $E_{6}$ of case (3) in Theorem 4.2.

Consider $A_{* 1}$ and $A_{* 2}$ attaching on $E_{6}$ in the following form:

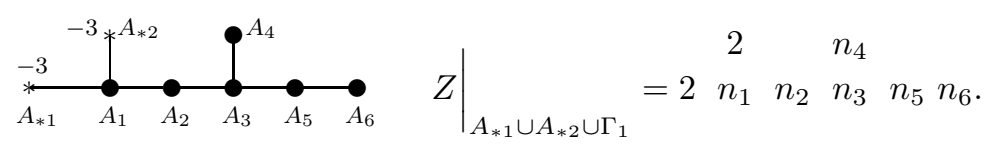

As in the proof of Proposition 6.11, we find out that this case is not possible. Consider $A_{* 1}$ and $A_{* 2}$ attaching on $E_{6}$ in the following form:

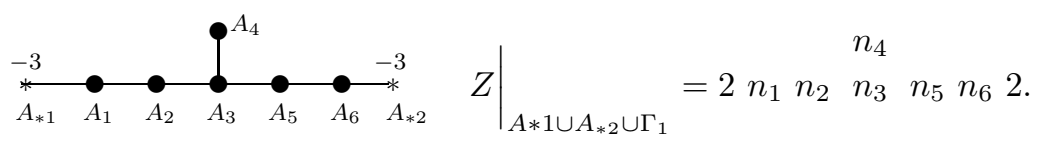


By the same argument as before, we have the following equations:

$$
\left\{\begin{array}{l}
-2 n_{1}+2+n_{2}=0 \\
-2 n_{2}+n_{1}+n_{3}=0 \\
-2 n_{3}+n_{2}+n_{4}+n_{5}=0 \\
-2 n_{4}+n_{3}=0 \\
-2 n_{5}+n_{3}+n_{6}=0 \\
-2 n_{6}+n_{5}+2=0 .
\end{array}\right.
$$

An easy exercise shows that we are in case (14).

(IV) Assume that $\Gamma_{1}$ is of the form $E_{7}$ of case (4) in Theorem 4.2 , Consider $A_{* 1}$ and $A_{* 2}$ attaching on $E_{7}$ in the following form:

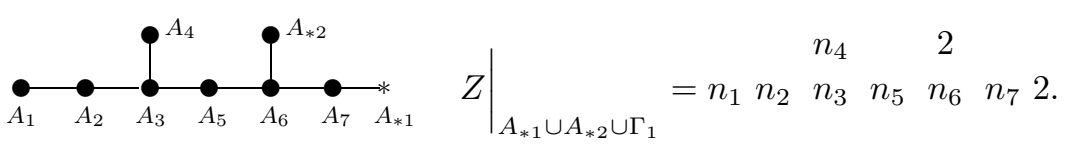

By the same argument as before, we have the following equations:

$$
\left\{\begin{array}{l}
-2 n_{1}+n_{2}=0 \\
-2 n_{2}+n_{1}+n_{3}=0 \\
-2 n_{3}+n_{2}+n_{4}+n_{5}=0 \\
-2 n_{4}+n_{3}=0 \\
-2 n_{5}+n_{3}+n_{6}=0 \\
-2 n_{6}+n_{5}+n_{7}=0 \\
-2 n_{7}+n_{6}+4=0 .
\end{array}\right.
$$

(6.11) implies

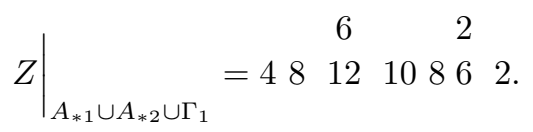

Since $-1=A_{* 1}^{2}+2=A_{* 1} \cdot(-K)=A_{* 1} \cdot Z \geq 2(-3)+6=0$, this is absurd. This case is impossible.

(V) Assume that $\Gamma_{1}$ is of the form $E_{8}$ of case (5) in Theorem 4.2. This case cannot happen because $A_{* 1} \cdot Z_{1} \geq 2$.

Theorem 6.19. Let $(V, p)$ be a germ of minimally elliptic singularity. Let $\pi: M \rightarrow$ $V$ be the minimal resolution of p. If case (4) of Proposition 6.2 holds, i.e., if there exist two effective components $A_{* 1}$ and $A_{* 2}$ with $A_{* 1}^{2}=-3=A_{* 2}^{2}$ and $z_{* 1}=2=$ $z_{* 2}$, then the weighted dual graph $\Gamma$ of the exceptional set is one of the following forms.

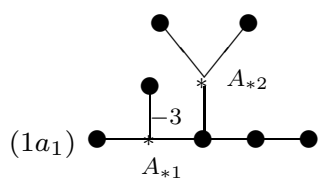

$$
\begin{aligned}
& 11 \\
& 1 \quad \underline{2} \\
& Z=1 \underline{2} \quad \begin{array}{lll}
3 & 21
\end{array}
\end{aligned}
$$


$\left(1 a_{2}\right)$

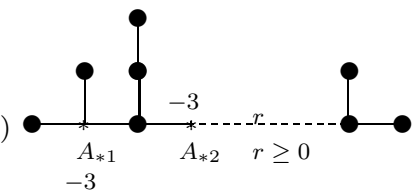

$(1 b)$

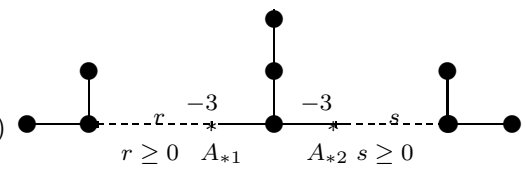

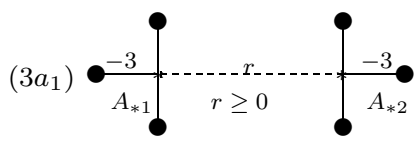

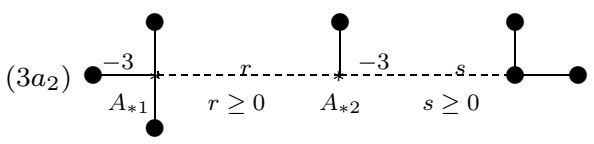

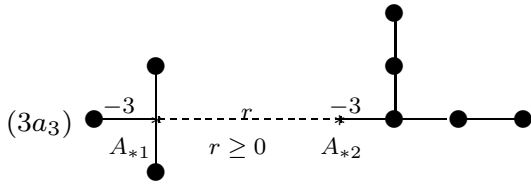

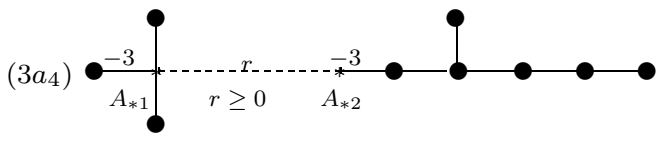

$\left(3 a_{5}\right)$

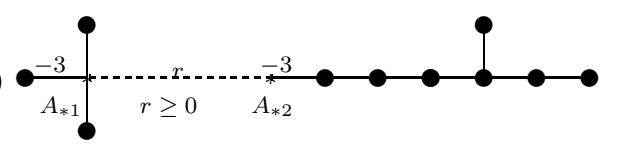

$\left(3 b_{1}\right)$

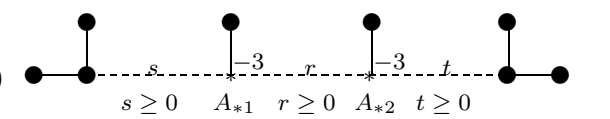

$\left(3 b_{2}\right)$

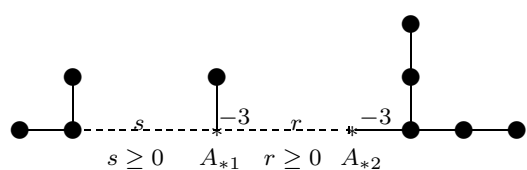

$\left(3 b_{3}\right)$

$\left(3 b_{4}\right)$

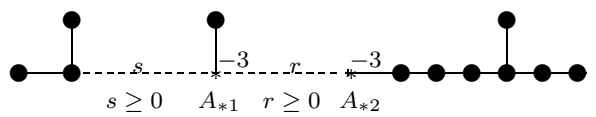

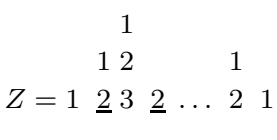

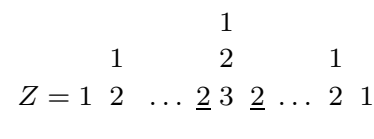

$Z=1 \begin{array}{cccc}1 & & 1 \\ \frac{2}{1} & \cdots & \frac{2}{1} & 1\end{array}$

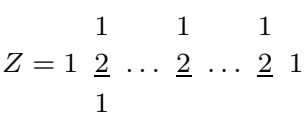

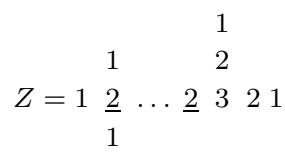

$1 \quad 2$

$Z=1 \underline{2} \ldots \underline{2} 34321$

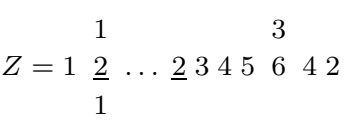

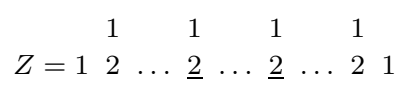

\begin{tabular}{lllllllll} 
& & & & & \multicolumn{3}{c}{1} & \\
& 1 & & & & 2 & & \\
1 & 2 & $\ldots$ & $\underline{2}$ & $\cdots$ & $\underline{2}$ & 3 & 2 & 1
\end{tabular}

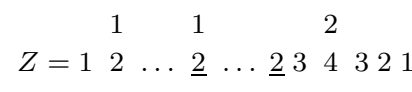

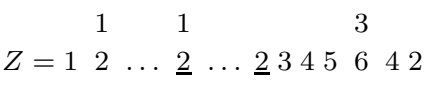


$\left(3 c_{1}\right)$

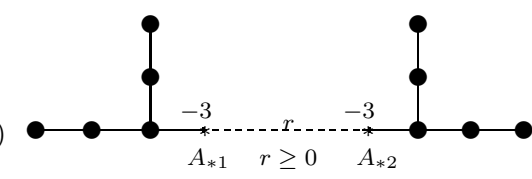

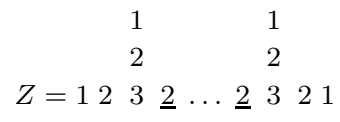

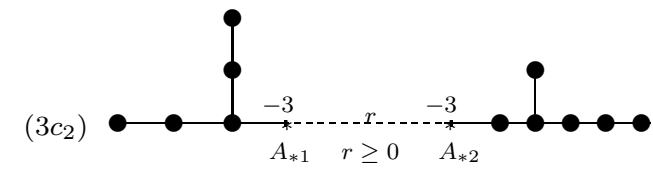

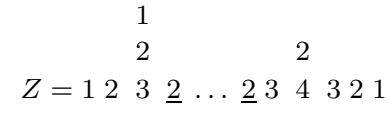

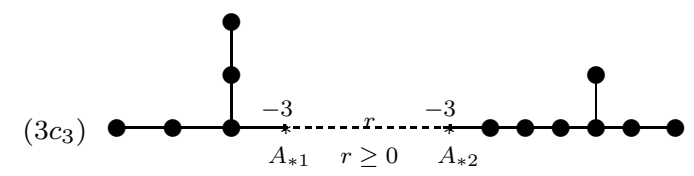

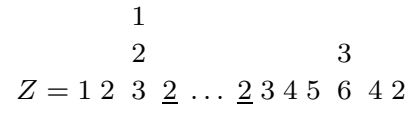

$\left(3 d_{1}\right)$

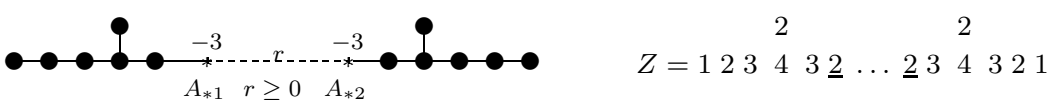

$\left(3 d_{2}\right)$

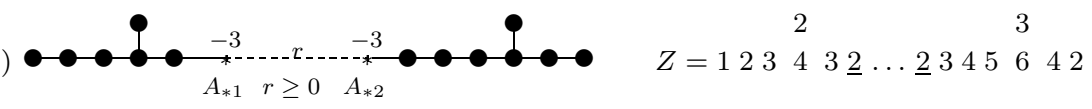

$(3 e)$

$\longrightarrow-\longrightarrow-3-3=246543 \underline{2} \ldots \underline{2} 345642$
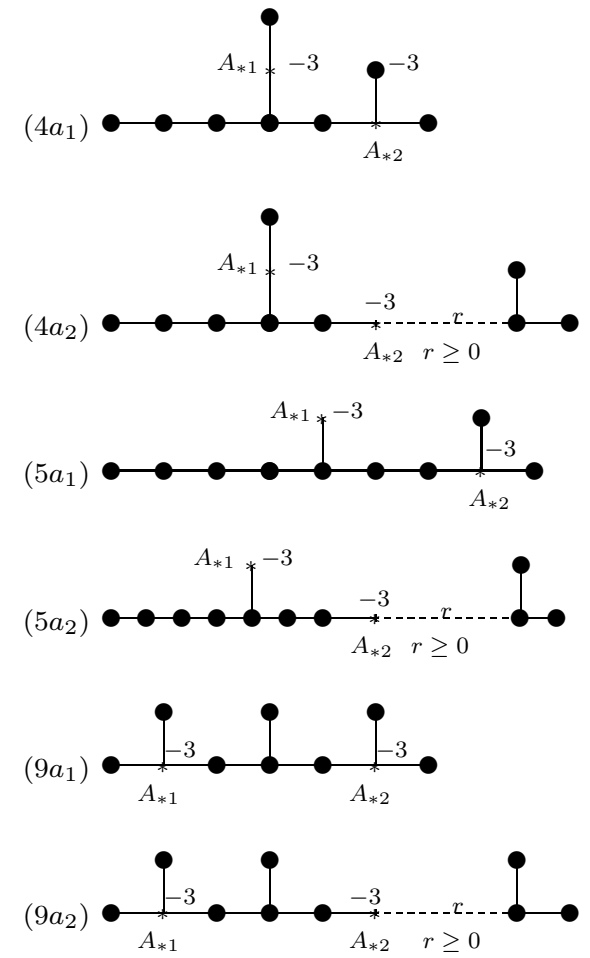

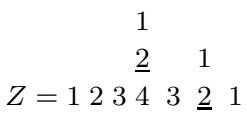

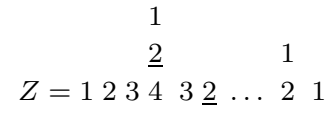

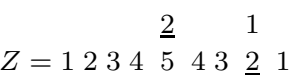

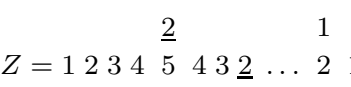

$$
\begin{aligned}
& 21 \\
& Z=1 \underline{2} \quad 3 \quad 4 \quad 3 \quad \underline{2} 1
\end{aligned}
$$

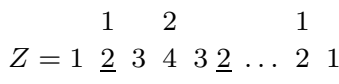


$(9 b)$

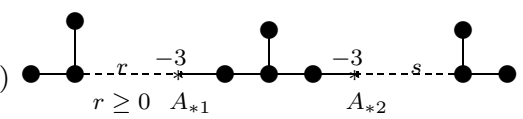

$\left(12 a_{1}\right)$

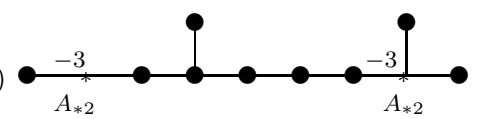

$\left(12 a_{2}\right)$

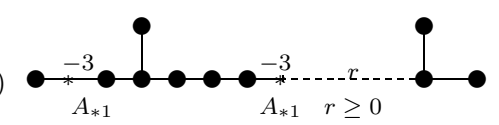

$\left(13 a_{1}\right)$
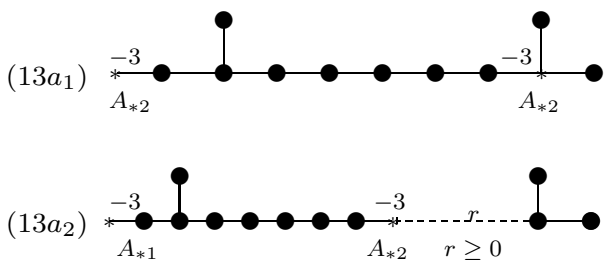

$$
\begin{aligned}
& Z=1 \quad \begin{array}{ccccccc}
1 & & 2 & & 1 \\
2 & 2 & 4 & 3 & \underline{2} & \ldots & 2
\end{array} \\
& Z=1 \underline{2} \begin{array}{rrrrr}
3 & & 1 \\
6 & 54 & 4 & \underline{2} & 1
\end{array} \\
& Z=1 \underline{2} 4 \begin{array}{l}
3 \\
6
\end{array} 543 \underline{2} \ldots 2 \quad \begin{array}{l}
1 \\
2
\end{array}
\end{aligned}
$$

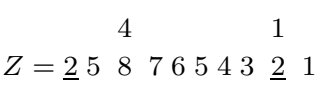

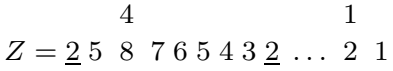

Proof. Since the singularity is minimally elliptic, $A_{* i}^{2}=-3, z_{* i}=2$ for $i=1$, 2 , we have

$$
A_{* i} \cdot\left(Z-2 A_{* i}\right)=-A_{* i} \cdot\left(K+2 A_{* i}\right)=A_{* i}^{2}+2-2 A_{* i}^{2}=5 .
$$

Let $\Gamma^{\prime}$ be the graph obtained by deleting $A_{* 1}$ and $A_{* 2}$ from $\Gamma$. Let $\Gamma_{1}, \ldots, \Gamma_{m}$ be the connected components of $\Gamma^{\prime}$ with fundamental cycles $Z_{1}, \ldots, Z_{m}$ respectively. (6.42) implies that

$$
\sum_{j=1}^{m} A_{* i} \cdot Z / \Gamma_{j}=5, \quad \text { for } i=1,2 .
$$

Since we have two effective components, by Corrollay 6.4 we have

$$
A_{* i} \cdot Z_{j}=1 \quad \text { for } i=1,2 \text { and } 1 \leq j \leq m .
$$

Consider first that $A_{* 1}$ and $A_{* 2}$ do not meet. Then Proposition 6.18 applies. For case (1) of Proposition 6.18 if the decomposition (6.43) at $A_{* 1}$ is $5=1+1+3$ and the decomposition (6.43) of $A_{* 2}$ is $5=1+1+3$, then we are in case $\left(1 a_{1}\right)$. If the decomposition (6.43) at $A_{* 1}$ is $5=1+1+3$ and the decomposition of (6.43) at $A_{* 2}$ is $2+3$, then we are in case $1\left(a_{2}\right)$. If the decomposition (6.43) at $A_{* 1}$ and $A_{* 2}$ are $2+3$, then we are in case $(1 b)$.

For case (2) of Proposition 6.18, the decomposition of (6.43) at $A_{* 1}$ and $A_{* 2}$ must be $5=4+1$. From Proposition 6.17, we obtain a possible dual graph together with a proposed fundamental cycle. One may check that the proposed fundamental cycle does not meet the minimum condition required by Definition 2.1. Therefore there is no dual graph produced from this case.

For case (3) of Proposition 6.18, if the decomposition of (6.43) at $A_{* 1}$ is $5=$ $2+1+1+1$ and the decomposition at $A_{* 2}$ is either $5=2+1+1+1$, or $5=2+2+1$, or $5=2+3$, according to Proposition 6.17 , we are in case $\left(3 a_{1}\right), \ldots,\left(3 a_{5}\right)$ respectively. 
If the decomposition of (6.43) at $A_{* 1}$ is $5=2+2+1$ and the decomposition at $A_{* 2}$ is $5=2+2+1$, or $5=2+3$, we are in case $\left(3 b_{1}\right), \ldots,\left(3 b_{4}\right)$. If the decompositions of $(6.43)$ at $A_{* 1}$ and at $A_{* 2}$ are both $5=2+3$, we are in case $\left(3 c_{1}\right),\left(3 c_{2}\right),\left(3 c_{3}\right)$, $\left(3 d_{1}\right),\left(3 d_{2}\right)$, and $(3 e)$.

For case (4) of Proposition 6.18, if the decomposition of (6.43) at $A_{* 1}$ is $5=4+1$ and the decomposition of (6.43) at $A_{* 2}$ is $5=3+1+1$, we have case $4\left(a_{1}\right)$. If the decompositions of (6.43) at $A_{* 1}$ and $A_{* 2}$ are $5=4+1$ and $5=3+2$ respectively, we have case $4\left(a_{2}\right)$.

For case (5) of Proposition 6.18, the decomposition of (6.43) at $A_{* 1}$ must be $5=5+0$. If the decomposition of $(6.43)$ at $A_{* 2}$ is $5=3+1+1$, we have case $5\left(a_{1}\right)$. If the decomposition of (6.43) at $A_{* 2}$ is $5=3+2$, we have case $5\left(a_{2}\right)$.

For case (6) of Proposition 6.18, the decompositions of (6.43) at $A_{* 1}$ and $A_{* 2}$ must be $5=4+1$. For case (7) of Proposition 6.18, the decomposition of (6.43) at $A_{* 1}$ and $A_{* 2}$ must be $5=5+0$. For case (8) of Proposition 6.18, the decompositions of (6.43) at $A_{* 1}$ and $A_{* 2}$ must be $5=4+1$. In all these cases, the proposed fundamental cycles on the possible dual graphs obtained via Proposition 6.17 do not meet the minimum condition required in Definition 2.1. Therefore there is no dual graph produced from these cases.

For case (9) of Proposition 6.18, if the decomposition of (6.43) at $A_{* 1}$ is $5=$ $3+1+1$ and the decomposition of $(6.43)$ at $A_{* 2}$ is $5=3+1+1$ or $5=3+2$, we have cases $9\left(a_{1}\right)$ and $9\left(a_{2}\right)$ respectively. If the decomposition of $(6.43)$ at $A_{* 1}$ and $A_{* 2}$ is $5=3+2$, we have case $9(b)$.

For case (10) of Proposition 6.18, the decomposition of (6.43) at $A_{* 1}$ and $A_{* 2}$ must be $5=4+1$. For case (11) of Proposition 6.18, the decomposition of (6.43) at $A_{* 1}$ and $A_{* 2}$ must be $5=5+0$. The proposed fundamental cycles on the possible dual graphs obtained via Proposition 6.17 do not meet the minimum condition of Definition 2.1. Hence there is no dual graph produced from these two cases.

For case (12) of Proposition 6.18, the decomposition of (6.43) at $A_{* 1}$ must be $5=4+1$. The decomposition of $(6.43)$ at $A_{* 2}$ must be $5=3+1+1$ or $5=3+2$. We have cases $12\left(a_{1}\right)$ and $12\left(a_{2}\right)$ respectively.

For case (13) of Proposition 6.18, the decomposition of (6.43) at $A_{* 1}$ must be $5=5+0$. The decomposition of $(6.43)$ at $A_{* 2}$ must be $5=3+1+1$ or $5=3+2$. We have cases $13\left(a_{1}\right)$ and $13\left(a_{2}\right)$ respectively.

For case (14) of Proposition 6.18, the decomposition of (6.43) at $A_{* 1}$ and $A_{* 2}$ must be $5=4+1$. Again the proposed fundamental cycle does not meet the minimum condition of Definition 2.1. There is no dual graph produced from this case.

We next consider the case $A_{* 1} \cdot A_{* 2}>0$. Since the singularity is minimally elliptic and $z_{* 1}=z_{* 2}=2$, it follows that $A_{* 1} \cdot A_{* 2}=1$. Then we are in cases $\left(3 a_{1}\right)-(3 e)$ by an argument similar to the above.

Proposition 6.20. Let $\Gamma$ be the minimal resolution graph of a minimally elliptic singularity with fundamental cycle $Z$. Let $\Gamma^{\prime}$ be the subgraph of $\Gamma$ obtained by removing all the effective components of $\Gamma$. Let $A_{*}$ be an effective component of $\Gamma$. Suppose that $\Gamma_{1}$ is a connected component of $\Gamma^{\prime}$ which corresponds to a rational double point graph in Theorem 4.2. Suppose also that $\Gamma_{1}$ intersects with $A_{*}$ but is disjoint from other effective components. Let $Z_{1}$ be the fundamental cycle on $\Gamma_{1}$. Suppose $A_{*} \cdot Z_{1}=1$. If the coefficient $z_{*}$ of $A_{*}$ in $Z$ is 3 and $A_{*}^{2}=-3$, then $A_{*} \cup \Gamma_{1}$ and the restriction of $Z$ on $A_{*} \cup \Gamma_{1}$ must be one of the following forms. 
(1)

$A_{*}^{*}$

(2)

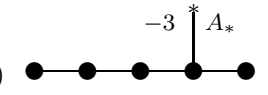

(3)

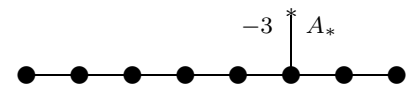

(4)

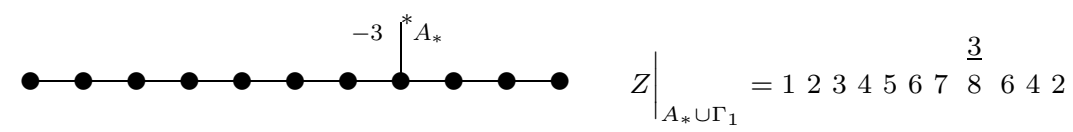

(5)

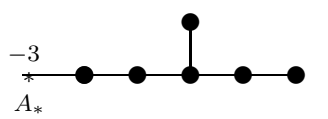

$\left.Z\right|_{A_{*} \cup \Gamma_{1}}=\underline{3} 21$

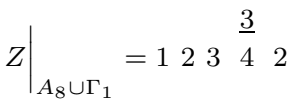

$\left.Z\right|_{A_{*} \cup \Gamma_{1}}=12345 \begin{aligned} & \underline{3} \\ & 6\end{aligned} 42$

$\left.Z\right|_{A_{*} \cup \Gamma_{1}}=12345678 \frac{3}{8} 642$

$\left.Z\right|_{A_{*} \cup \Gamma_{1}}=\underline{3} 456 \begin{array}{lll}3 \\ 6 & 4 & \end{array}$

Proof. The proof is similar to those of Propositions 6.9, 6.10 and 6.11

Proposition 6.21. Let $\Gamma$ be the minimal resolution graph of a minimally elliptic singularity with fundamental cycle $Z$. Let $\Gamma^{\prime}$ be the subgraph of $\Gamma$ obtained by removing all the effective components of $\Gamma$. Let $A_{*}$ be an effective component of $\Gamma$. Suppose that $\Gamma_{1}$ is a connected component of $\Gamma^{\prime}$ which corresponds to a rational double point graph in Theorem 4.2. Suppose also that $\Gamma_{1}$ intersects with $A_{*}$, but is disjoint from other effective components. Let $Z_{1}$ be the fundamental cycle on $\Gamma_{1}$. Suppose $A_{*} \cdot Z_{1}=1$. If the coefficient $z_{*}$ of $A_{*}$ in $Z$ is 1 and $A_{*}^{2} \leq-3$, then such a graph does not exist.

Proof. The proof is similar to those of Propositions 6.9, 6.10, and 6.11,

Proposition 6.22. Let $\Gamma$ be the minimal resolution graph of a minimally elliptic singularity with fundamental cycle $Z$. Let $\Gamma^{\prime}$ be the subgraph of $\Gamma$ obtained by removing all the effective components of $\Gamma$. Let $A_{* 1}$ and $A_{* 2}$ be two effective components of $\Gamma$. Suppose that $\Gamma_{1}$ is a connected component of $\Gamma^{\prime}$ which corresponds to a rational double point graph in Theorem 4.2. Suppose also that $\Gamma_{1}$ intersects with $A_{* 1}$ and $A_{* 2}$, but is disjoint from other effective components. Let $Z_{1}$ be the fundamental cycle on $\Gamma_{1}$. Suppose $A_{* 1} \cdot Z_{1}=1=A_{* 2} \cdot Z_{1}$. If $A_{* 1} \cdot A_{* 2}=0$, the coefficients $z_{* 1}$ and $z_{* 2}$ of $A_{* 1}$ and $A_{* 2}$ are 3 and 1 respectively, and $A_{* 1}^{2}=A_{* 2}^{2}=-3$, then $A_{* 1} \cup A_{* 2} \cup \Gamma_{1}$ and the restriction of $Z$ on $A_{* 1} \cup A_{2} \cup \Gamma_{1}$ must be one of the following forms.

(1)

(2)
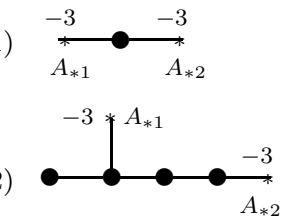

$\left.Z\right|_{A_{* 1} \cup A_{* 2} \cup \Gamma_{1}}=\underline{3} 2 \underline{1}$

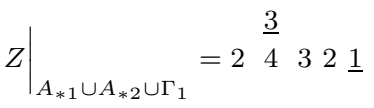


(3)

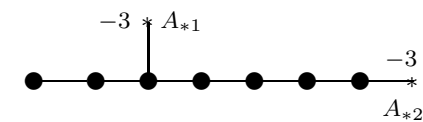

(4)

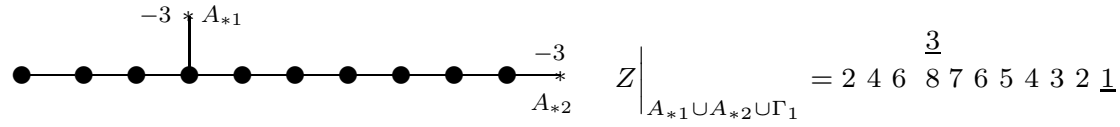

Proof. The proof is the same as those in Proposition 6.18.

Theorem 6.23. Let $(V, p)$ be a germ of minimally elliptic singularity. Let $\pi: M \rightarrow$ $V$ be the minimal resolution of $p$. If case (5) of Proposition 6.2 holds, i.e., if there exist two effective components $A_{* 1}$ and $A_{* 2}$ with $A_{* 1}^{2}=-3=A_{* 2}^{2}$ and $z_{* 1}=3$, $z_{* 2}=1$, then the weighted dual graph $\Gamma$ of the exceptional set is one of the following forms.

$(1 a)$

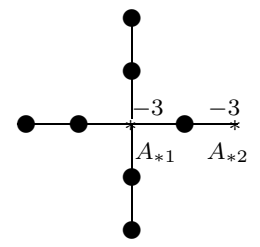

$\left(1 b_{1}\right)$

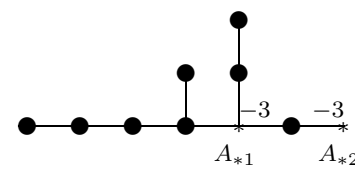

$\left(1 b_{2}\right)$

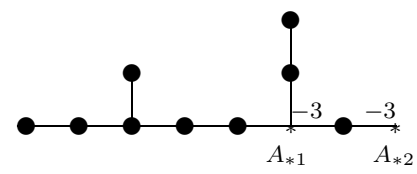

$(1 c)$

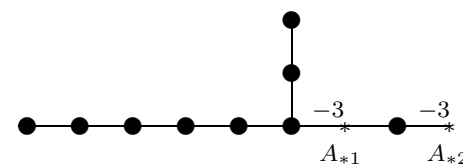

$(2 a)$

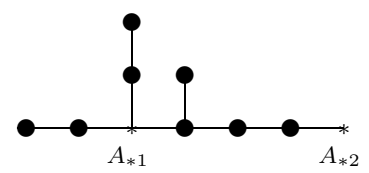

$\left(2 b_{1}\right)$

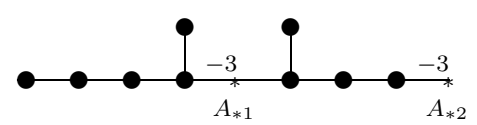

$\left(2 b_{2}\right)$

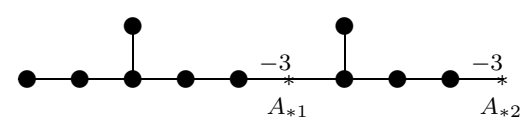

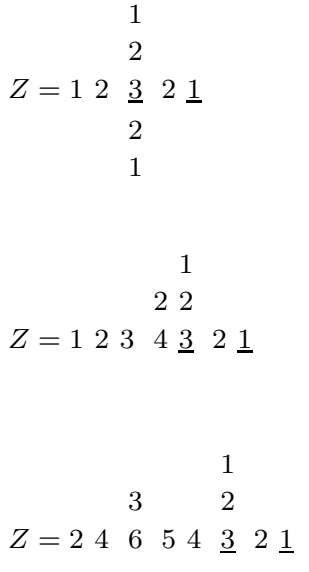

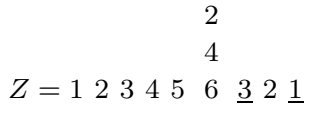

22

$Z=12 \underline{3} 4 \quad 3 \quad 2 \underline{1}$

$2 \quad 2$

$Z=12234 \underline{3} 4 \quad 3 \quad 2 \underline{1}$

$3 \quad 2$

$Z=24 \quad 6 \quad 54 \underline{3} 4 \begin{array}{llll}3 & 2 & 1\end{array}$ 
(3)

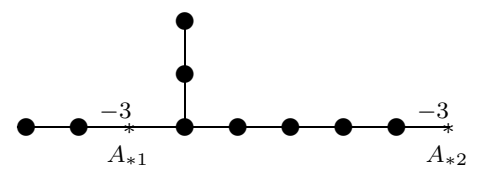

(4)

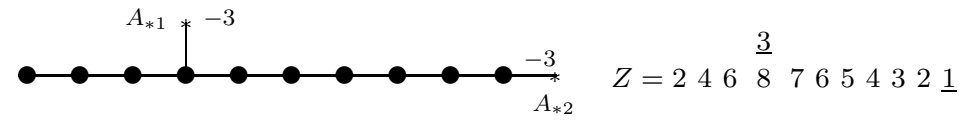

Proof. Since $z_{* 2}=1, z_{* 1}=3, A_{* 2}^{2}=-3$ and $A_{* 2} \cdot Z=-1$, we have $A_{* 1} \cdot A_{* 2}=0$. The proof is similar to those of Theorem 6.19 by using Propositions 6.20, 6.21, and 6.22 .

Proposition 6.24. Let $\Gamma$ be the minimal resolution graph of a minimally elliptic singularity with fundamental cycle $Z$. Let $\Gamma^{\prime}$ be the subgraph of $\Gamma$ obtaeind by removing all the effective components of $\Gamma$. Let $A_{* 1}$ and $A_{* 2}$ be two effective components of $\Gamma$. Suppose that $\Gamma_{1}$ is a connected component of $\Gamma^{\prime}$ which corresponds to a rational double point graph in Theorem 4.2. Suppose also that $\Gamma_{1}$ intersects with $A_{* 1}$ and $A_{* 2}$, but is disjoint from other effective components. Let $Z_{1}$ be the fundamental cycle on $\Gamma_{1}$. Suppose that $A_{* 1} \cdot Z_{1}=1=A_{* 2} \cdot Z_{1}$. If $A_{* 1} \cdot A_{* 2}=0$, $z_{* 1}=2, z_{* 2}=1$ (coefficients of $A_{* 1}$ and $A_{* 2}$ in $Z$ respectively), and $A_{* 1}^{2}=-3$, $A_{* 2}^{2}=-4$ or -3 , then $A_{* 1} \cup A_{* 2} \cup \Gamma_{1}$ and the restriction of $Z$ on $A_{* 1} \cup A_{* 2} \cup \Gamma_{1}$ must be one of the following forms. (In case $A_{* 2}^{2}=-3$, replace -4 by -3 , in the following graphs.)

(1)

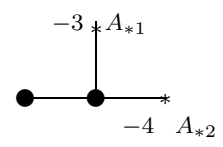

(2)

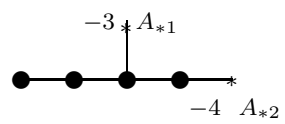

(3)

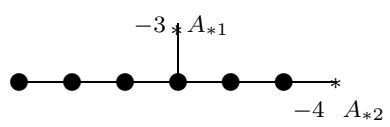

(4)

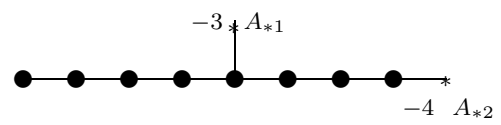

(5)

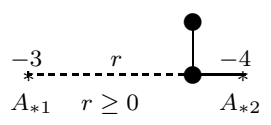

(6)

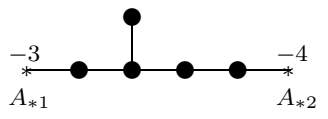

$$
\begin{aligned}
& \left.Z\right|_{A_{* 1} \cup A_{* 2} \cup \Gamma_{1}}=1 \frac{\underline{2}}{2} \underline{1} \\
& \left.Z\right|_{A_{* 1} \cup A_{* 2} \cup \Gamma_{1}}=12 \frac{\underline{2}}{3} 2 \underline{1}
\end{aligned}
$$$$
\left.Z\right|_{A_{* 1} \cup A_{* 2} \cup \Gamma_{1}}=123 \quad \underline{2} \quad 3 \quad 32 \underline{1}
$$$$
\left.Z\right|_{A_{* 1} \cup A_{* 2} \cup \Gamma_{1}}=12345 \underline{5} 432 \underline{\underline{2}}
$$$$
\left.Z\right|_{A_{* 1} \cup A_{* 2} \cup \Gamma_{1}}=\underline{2} \ldots \ldots \quad \begin{gathered}
1 \\
2
\end{gathered}
$$

2

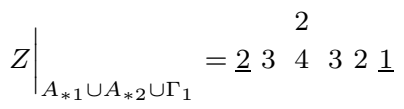


(7)

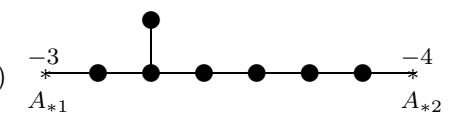

(8)

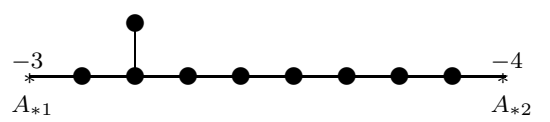

$$
\begin{aligned}
& \left.Z\right|_{A_{* 1} \cup A_{* 2} \cup \Gamma_{1}}=\underline{2} 4 \quad 6 \quad 6 \quad 5432 \underline{1} \\
& \left.Z\right|_{A_{* 1} \cup A_{* 2} \cup \Gamma_{1}}=\underline{2} 5 \quad 8 \quad 8 \quad 7655432 \underline{1}
\end{aligned}
$$

Proof. The proof is the same as that in Proposition 6.22.

Theorem 6.25. Let $(V, p)$ be a germ of minimally elliptic singularity. Let $\pi: M \rightarrow$ $V$ be the minimal resolution of p. If case (6) of Proposition 6.2 holds, i.e., if there exist two effective components $A_{* 1}$ and $A_{* 2}$ with $A_{* 1}^{2}=-3, A_{* 2}^{2}=-4$ and $z_{* 1}=2$, $z_{* 2}=1$, then the weighted dual graph $\Gamma$ of the exceptional set is one of the following forms.

(1a)

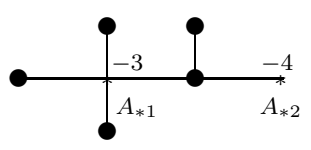

(1b)

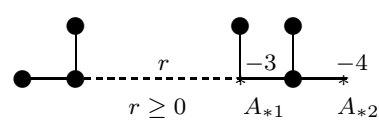

$\left(1 c_{1}\right)$

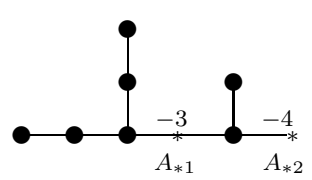

$\left(1 c_{2}\right)$

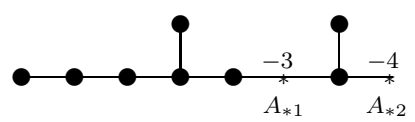

$\left(1 c_{3}\right)$

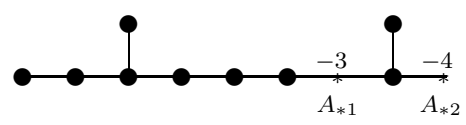

(2a)

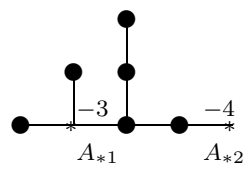

(2b)

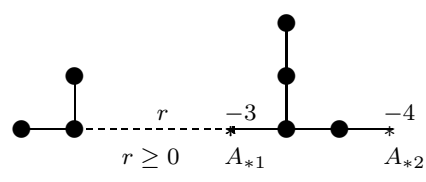

11

$Z=1 \underline{2} 2 \underline{1}$

1

$Z=\begin{array}{llllll} & 1 & & 1 & 1 \\ 1 & 2 & \ldots & \underline{2} & 2 & \underline{1}\end{array}$

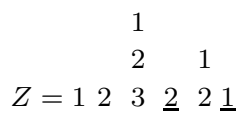

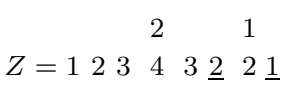

$3 \quad 1$

$Z=24 \quad 6 \quad 543 \underline{2} 2 \underline{1}$

12

$Z=1 \underline{2} 3 \quad 2 \underline{1}$

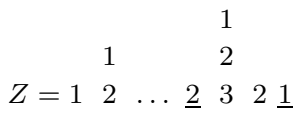


$(3 \mathrm{a})$

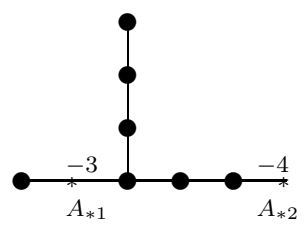

(4)

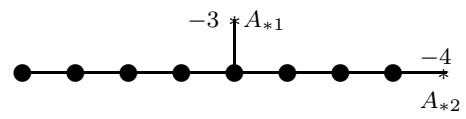

(5a)

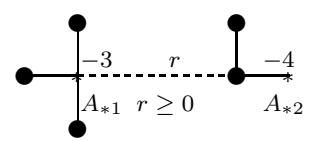

$(5 \mathrm{~b})$

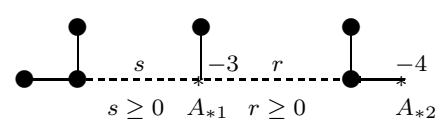

$\left(5 c_{1}\right)$

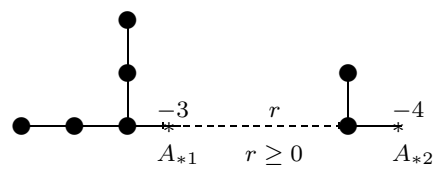

$\left(5 c_{2}\right)$

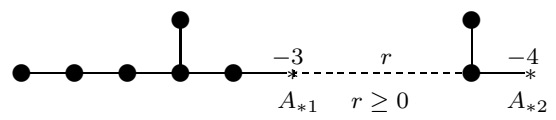

$\left(5 c_{3}\right)$

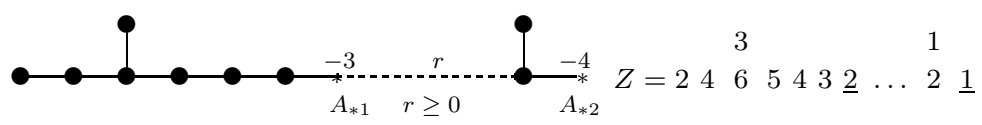

(6a)

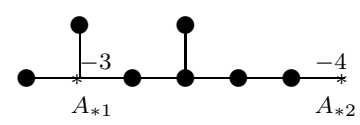

(6b)

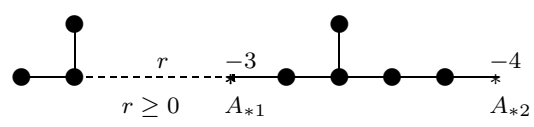

(7a)

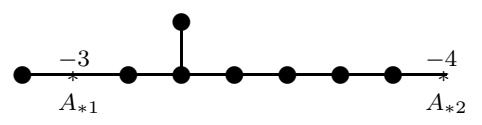

(8)

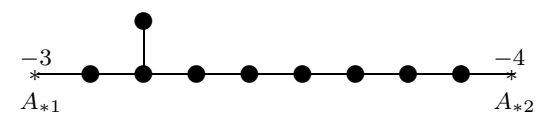

(9)

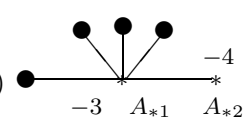

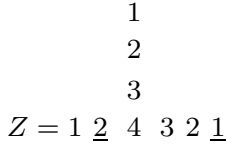

$Z=12234$\begin{tabular}{l}
$\underline{2}$ \\
\hline
\end{tabular}

$Z=1 \quad \begin{array}{lllll}1 & & 1 & \\ \underline{2} & \ldots & 2 & \underline{1} \\ 1 & & & \end{array}$

$\begin{array}{lllllll} & & & 1 & & 1 \\ & & & & & & \\ 2 & 2 & \ldots & 2 & \underline{1}\end{array}$

$\begin{array}{ll}1 & \\ 2 & 1\end{array}$

$Z=12 \quad 3 \quad 2 \quad \ldots \quad 2 \quad \underline{1}$

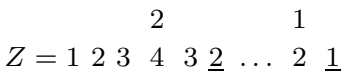

$Z=1 \quad \begin{array}{llllll}1 & & 2 & & \\ & \underline{2} & 3 & 4 & 3 & 2\end{array}$

$Z=$\begin{tabular}{lllllllll} 
& 1 & \multicolumn{9}{c}{2} \\
$Z$ & 2 & $\ldots$ & $\underline{2}$ & 3 & 4 & 3 & 2 & 1
\end{tabular}

3

$Z=1 \underline{2} 4 \quad 6 \quad 5432 \underline{1}$

4

$Z=\underline{2} 587765432 \underline{1}$

$Z=1 \quad \begin{array}{lll}1 & 1 & 1 \\ 2 & \underline{1}\end{array}$ 
(10)

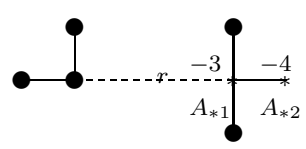

(11)

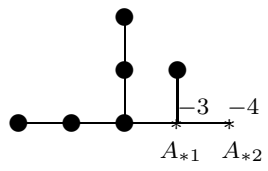

(12)

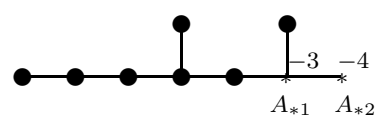

(13)

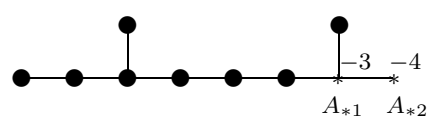

(14)

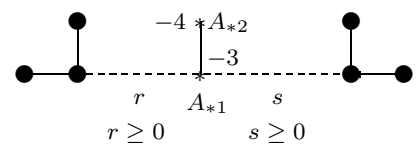

(15)

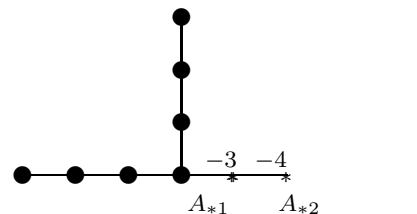

(16)

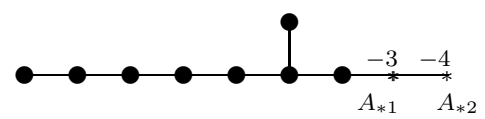

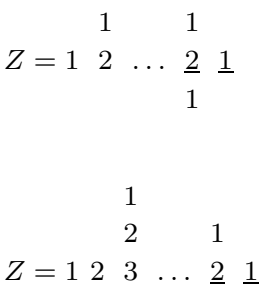

21

$Z=123 \quad 4 \quad 3 \quad \underline{2} \underline{1}$

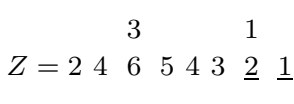

$Z=\begin{array}{llllllll} & 1 & & \underline{1} & & 1 & \\ 2 & 2 & \ldots & \underline{2} & \ldots & 2 & 1\end{array}$

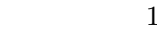

1

3

$Z=123 \quad 4 \quad \underline{2} \underline{1}$

3

$Z=12345 \quad 6 \quad 4 \underline{2} \underline{1}$

Proof. If $A_{* 1} \cdot A_{* 2}=0$, then the proof is similar to that of Theorem 6.23 by using Proposition 6.21, Proposition 6.24 and Proposition 6.17. We have case (1a) to case (8).

If $A_{* 1} \cdot A_{* 2} \neq 0$, then $A_{* 1} \cdot A_{* 2}=1$. It follows that $A_{* 1} \cdot\left(Z-2 A_{* 1}-A_{* 2}\right)=$ $-A_{* 1} \cdot\left(K+2 A_{* 1}+A_{* 2}\right)=-A_{* 1}^{2}+1=4$. For $4=1+1+1+1$, we are in case (9). For $4=1+1+2$, we are in case (10). For $4=1+3$, we are in case (11), case (12) and case (13). For $4=2+2$, we are in case (15). For $4=4$, we are in case (16) and case (17).

Proposition 6.26. Let $\Gamma$ be the minimal resolution graph of a minimally elliptic singularity with fundamental cycle $Z$. Let $\Gamma^{\prime}$ be the subgraph of $\Gamma$ obtained by removing all the effective components of $\Gamma$. Let $A_{* 1}$ and $A_{* 2}$ be two effective components of $\Gamma$. Suppose that $\Gamma_{1}$ is a connected component of $\Gamma^{\prime}$ which corresponds to a rational double point graph in Theorem 4.2. Suppose also that $\Gamma_{1}$ intersects 
with both $A_{* 1}$ and $A_{* 2}$, but with no other effective component. Let $Z_{1}$ be the fundamental cycle on $\Gamma_{1}$. Suppose $A_{* 1} \cdot Z_{1}=A_{* 2} \cdot Z_{1}=1$. If $A_{* 1} \cdot A_{* 2}=0$ and the coefficients $z_{* 1}$ of $A_{* 1}$ and $z_{* 2}$ of $A_{* 2}$ in $Z$ are one and $A_{* 1}^{2} \leq-3, A_{* 2}^{2} \leq-3$, then $A_{* 1} \cup A_{* 2} \cup \Gamma_{1}$ and the restriction of $Z$ on $A_{* 1} \cup A_{* 2} \cup \Gamma_{1}$ must be one of the following forms.

(1)

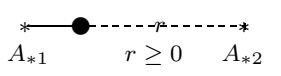

(2)

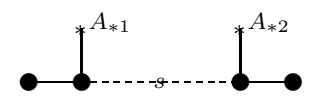

(3)

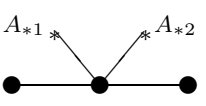

(4)

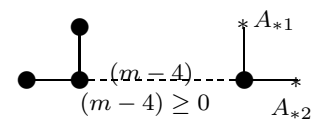

(5)

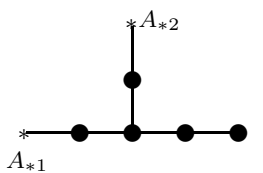

(6)

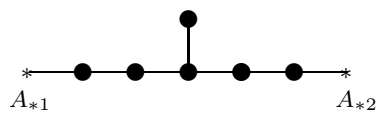

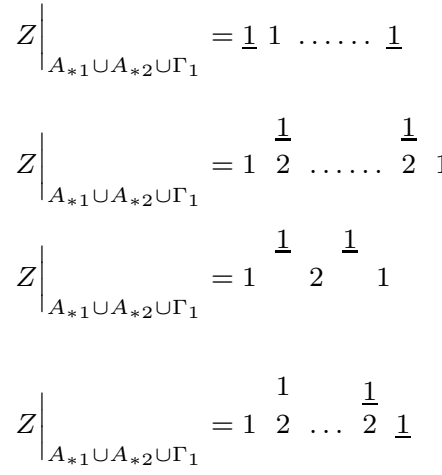

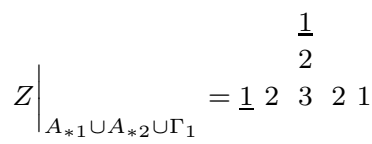

$\left.Z\right|_{A_{* 1} \cup A_{* 2} \cup \Gamma_{1}}=\underline{1} 2 \begin{array}{r}2 \\ 2\end{array}$

Proof. The proof is the same as that in Proposition 6.24.

Theorem 6.27. Let $(V, p)$ be a germ of minimally elliptic singularity. Let $\pi: M \rightarrow$ $V$ be the minimal resolution of p. If case (7) of Proposition 6.2 holds, i.e., if there exist two effective components $A_{* 1}$ and $A_{* 2}$ with $A_{* 1}^{2}=-4=A_{* 2}^{2}$ and $z_{* 1}=1=$ $z_{* 2}$, then the weighted dual graph $\Gamma$ of the exceptional set is one of the following forms.

(1)

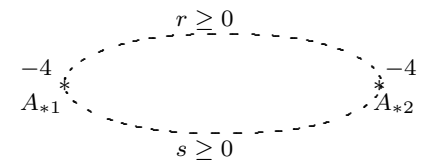

(2)

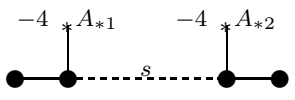

(3)

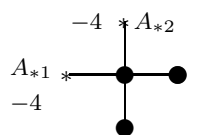

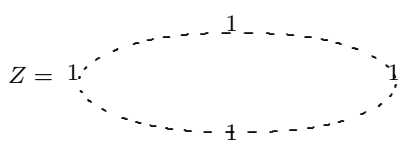

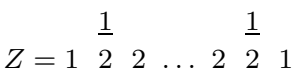

$$
Z=\underline{1} \begin{array}{ll}
\frac{1}{2} & 1 \\
1 &
\end{array}
$$


(4)

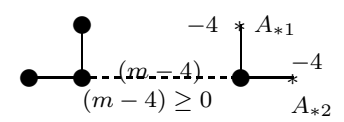

(5)

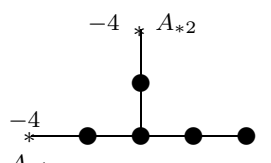

$A_{* 1}$

(6)

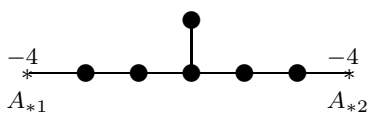

$Z=\begin{array}{lllll} & 1 & & \underline{1} \\ & 2 & \ldots & 2 & 1\end{array}$

$\frac{1}{2}$

$Z=\underline{1} 2 \begin{array}{llll}2 & 3 & 2 & 1\end{array}$

$Z=\underline{1} 234432 \underline{1}$

Proof. This follows from Proposition 6.3. Proposition 6.21 and Proposition 6.26.

Proposition 6.28. Let $\Gamma$ be the minimal resolution graph of a minimally elliptic singularity with fundamental cycle $Z$. Let $\Gamma^{\prime}$ be the subgraph of $\Gamma$ obtained by removing all the effective components of $\Gamma$. Let $A_{* 1}$ and $A_{* 2}$ be two effective components of $\Gamma$. Suppose that $\Gamma_{1}$ is a connected component of $\Gamma^{\prime}$ which corresponds to a rational double point graph in Theorem 4.2. Suppose also that $\Gamma_{1}$ intersects with $A_{* 1}$ and $A_{* 2}$, but is disjoint from other effective components. Let $Z_{1}$ be the fundamental cycle on $\Gamma_{1}$. Suppose that $A_{* 1} \cdot Z_{1}=1=A_{* 2} \cdot Z_{1}$. If $A_{* 1} \cdot A_{* 2}=0$, $z_{* 1}=1=z_{* 2}$, and $A_{* 1}^{2}=-3, A_{* 2}^{2}=-5$, then $A_{* 1} \cup A_{* 2} \cup \Gamma_{1}$ and the restriction of $Z$ on $A_{* 1} \cup A_{* 2} \cup \Gamma_{1}$ must be one of the following forms.

(1)

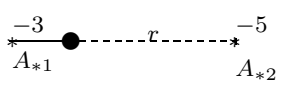

(2)

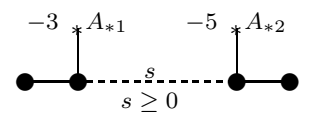

(3)

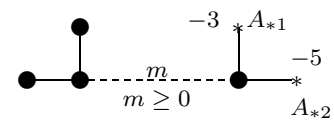

(4)

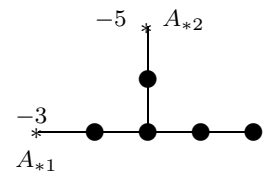

(5)

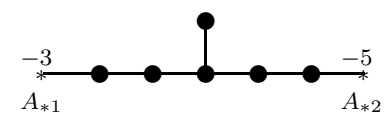

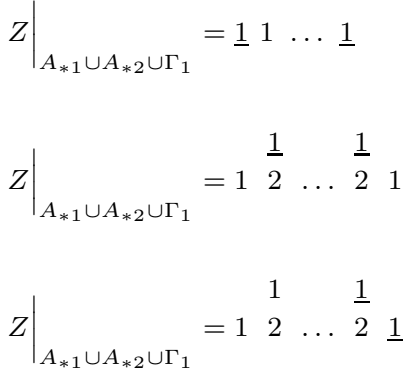

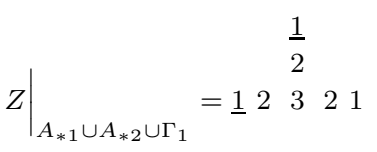

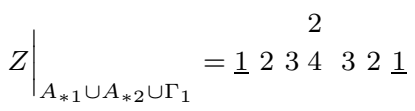


Proof. The proof is the same as that in Proposition 6.26.

Theorem 6.29. Let $(V, p)$ be a germ of minimally elliptic singularity. Let $\pi: M \rightarrow$ $V$ be the minimal resolution of $p$. If case (8) of Proposition 6.2 holds, i.e., if there exist two effective components $A_{* 1}$ and $A_{* 2}$ with $A_{* 1}^{2}=-3, A_{* 2}^{2}=-5$ and $z_{* 1}=z_{* 2}=1$, then the weighted dual graph $\Gamma$ of the exceptional set is one of the following forms.

(1)

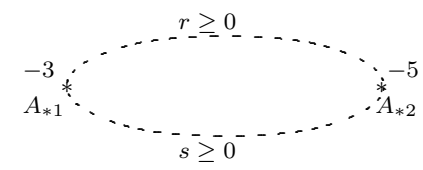

(2)

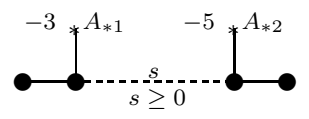

(3)

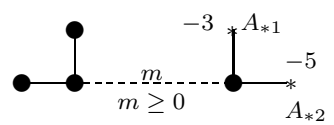

(4)

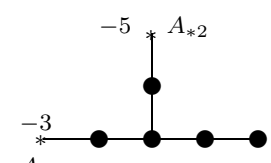

(5)

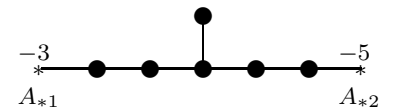

$Z=1:{ }_{1 \cdots}^{1 \cdots}$

$Z=1 \frac{1}{2} \quad \ldots \quad \frac{1}{2} \quad 1$

$Z=1 \quad 2 \quad \ldots \quad \frac{1}{2} \underline{1}$

$\frac{1}{2}$

$Z=\underline{1} 2 \begin{array}{llll}2 & 3 & 2\end{array}$

2

$Z=\underline{1} 233442 \underline{1}$

Proof. This follows from Proposition 6.3, Proposition 6.21 and Proposition 6.28.

Proposition 6.30. Let $\Gamma$ be the minimal resolution graph of a minimally elliptic singularity with fundamental cycle $Z$. Let $\Gamma^{\prime}$ be the subgraph of $\Gamma$ obtained by removing all the effective components of $\Gamma$. Let $A_{* 1}, A_{* 2}$ and $A_{* 3}$ be three effective components of $\Gamma$. Suppose that $\Gamma_{1}$ is a connected component of $\Gamma^{\prime}$ which corresponds to a rational double point graph in Theorem 4.2. Suppose also that $\Gamma_{1}$ intersects with $A_{* 1}, A_{* 2}$ and $A_{* 3}$, but is disjoint from other effective components. Let $Z_{1}$ be the fundamental cycle on $\Gamma_{1}$. Suppose that $A_{* 1} \cdot Z_{1}=1=A_{* 2} \cdot Z_{1}=A_{* 3} \cdot Z_{1}$. If $A_{* 1}, A_{* 2}$ and $A_{* 3}$ are mutually disjoint, $z_{* 1}=1=z_{* 2}, z_{* 3}=2$, and $A_{* 1}^{2}=-3=A_{* 2}^{2}=A_{* 3}^{2}$, then $A_{* 1} \cup A_{* 2} \cup A_{* 3} \cup \Gamma_{1}$ and the restriction of $Z$ on $A_{* 1} \cup A_{* 2} \cup A_{* 3} \cup \Gamma_{1}$ must be one of the following forms.

(1)

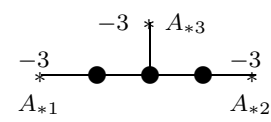

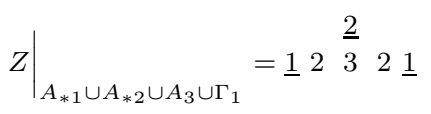


(2)

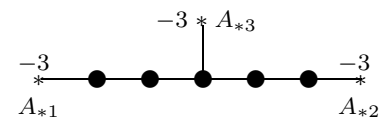

(3)

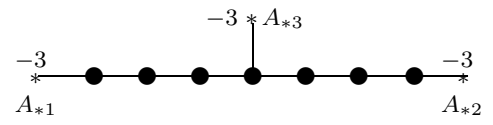

(4)

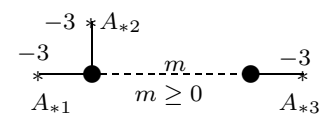

$\left.Z\right|_{A_{* 1} \cup A_{* 2} \cup A_{3} \cup \Gamma_{1}}=\underline{1} 2 \begin{array}{llll}\underline{2} & 4 & 3 & 2\end{array}$

$\left.Z\right|_{A_{* 1} \cup A_{* 2} \cup A_{3} \cup \Gamma_{1}}=\underline{1} 234 \frac{\underline{2}}{5} 432 \underline{1}$

$\left.Z\right|_{A_{* 1} \cup A_{* 2} \cup A_{3} \cup \Gamma_{1}}=\underline{1}{ }^{\underline{1}} \ldots 2 \underline{2}$

Proof. The proof is the same as that in Proposition 6.28.

Proposition 6.31. Let $\Gamma$ be the minimal resolution graph of a minimally elliptic singularity with fundamental cycle $Z$. Let $\Gamma^{\prime}$ be the subgraph of $\Gamma$ obtained by removing all the effective components of $\Gamma$. Let $A_{*}$ be an effective component of $\Gamma$. Suppose that $\Gamma_{1}$ is a connected component of $\Gamma^{\prime}$ which corresponds to a rational double point graph in Theorem 4.2. Suppose also that $\Gamma_{1}$ intersects with $A_{*}$ but is disjoint from other effective components. Let $Z_{1}$ be the fundamental cycle on $\Gamma_{1}$. Suppose $A_{*} \cdot Z_{1}=1$. If the coefficient $z_{*}$ of $A_{*}$ in $Z$ is 2 and $A_{*}^{2}=-3$, then $A_{*} \cup \Gamma_{1}$ and the restriction of $Z$ on $A_{*} \cup \Gamma_{1}$ must be one of the following forms.

(1)

(2)

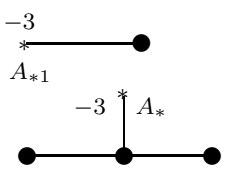

(3)

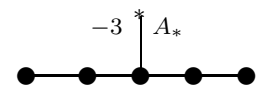

(4)

(5)

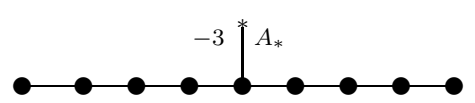

(6)

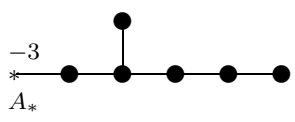

(7)

(8)
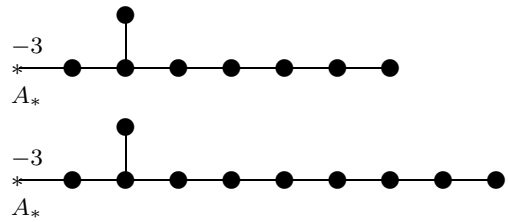

$\left.A\right|_{A_{*} \cup \Gamma_{1}}=\underline{2} 1$

$\left.A\right|_{A_{*} \cup \Gamma_{1}}=1 \frac{2}{2} 1$

$\left.A\right|_{A_{*} \cup \Gamma_{1}}=12 \begin{array}{llll}3 & 2 & 2 & \end{array}$

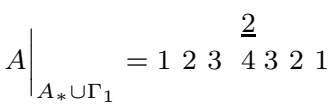

$\left.A\right|_{A_{*} \cup \Gamma_{1}}=1234 \frac{2}{5} 4321$

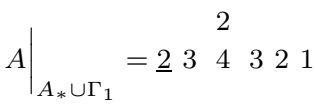

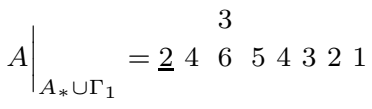

$\left.A\right|_{A_{*} \cup \Gamma_{1}}=\underline{2} 58 \begin{array}{r}4 \\ 8\end{array}$ 
(9)

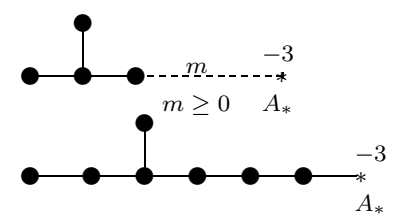

$$
\begin{aligned}
& \left.A\right|_{A_{*} \cup \Gamma_{1}}=1 \quad \begin{array}{ll}
1 & \\
& 2
\end{array} \ldots \underline{2} \\
& \left.A\right|_{A_{*} \cup \Gamma_{1}}=246543 \underline{2}
\end{aligned}
$$

Proof. The proof is the same as that in Proposition 6.13.

Proposition 6.32. Let $\Gamma$ be the minimal resolution graph of a minimally elliptic singularity with fundamental cycle $Z$. Let $\Gamma^{\prime}$ be the subgraph of $\Gamma$ obtained by removing all the effective components of $\Gamma$. Let $A_{* 1}$ and $A_{* 2}$ be two effective components of $\Gamma$. Suppose that $\Gamma_{1}$ is a connected component of $\Gamma^{\prime}$ which corresponds to a rational double point graph in Theorem 4.2, Suppose also that $\Gamma_{1}$ intersects with $A_{* 1}$ and $A_{* 2}$, but is disjoint from other effective components. Let $Z_{1}$ be the fundamental cycle on $\Gamma_{1}$. Suppose that $A_{* 1} \cdot Z_{1}=1=A_{* 2} \cdot Z_{1}$. If $A_{* 1} \cdot A_{* 2}=0, z_{* 1}=2$, $z_{* 2}=1$ (coefficient of $A_{* 1}$ and $A_{* 2}$ in $Z$ respectively), and $A_{* 1}^{2}=-3=A_{* 2}^{3}$, then $A_{* 1} \cup A_{* 2} \cup \Gamma_{1}$ and the restriction of $Z$ on $A_{* 1} \cup A_{* 2} \cup \Gamma_{1}$ must be one of the following forms.

(1)

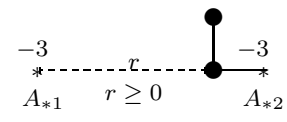

$(2)$

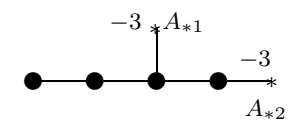

(3)

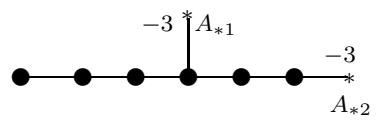

(4)

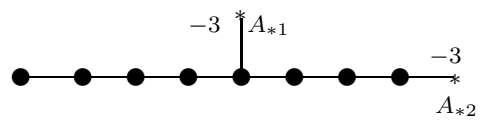

(5)

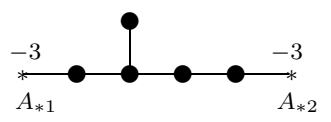

(6)

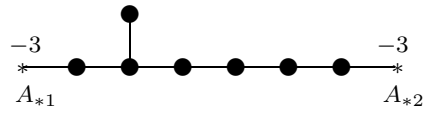

(7)

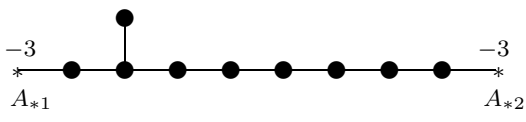

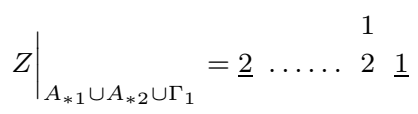

$$
\begin{aligned}
& \left.Z\right|_{A_{* 1} \cup A_{* 2} \cup \Gamma_{1}}=12 \stackrel{\underline{2}}{3} 2 \underline{1}
\end{aligned}
$$

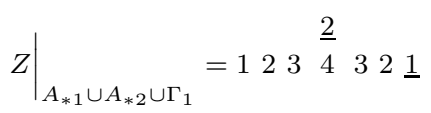$$
\left.Z\right|_{A_{* 1} \cup A_{* 2} \cup \Gamma_{1}}=1234 \stackrel{\underline{2}}{5} 432 \underline{1}
$$$$
\left.Z\right|_{A_{* 1} \cup A_{* 2} \cup \Gamma_{1}}=\underline{2} 3 \begin{array}{llll}
2 & 4 & 3 & 2
\end{array}
$$

$\left.Z\right|_{A_{* 1} \cup A_{* 2} \cup \Gamma_{1}}=\underline{2} 4 \begin{array}{llllll}3 & 6 & 5 & 4 & & \\ & & & \end{array}$

$\left.Z\right|_{A_{* 1} \cup A_{* 2} \cup \Gamma_{1}}=\underline{2} 5 \quad 8 \quad 7 \quad 765432 \underline{1}$

Proof. The proof is the same as that in Proposition 6.24. 
Theorem 6.33. Let $(V, p)$ be a germ of minimally elliptic singularity. Let $\pi: M \rightarrow$ $V$ be the minimal resolution of p. If case (9) of Proposition 6.2 holds, i.e., if there exist three effective components $A_{* 1}, A_{* 2}$ and $A_{* 3}$ with $A_{* 1}^{2}=A_{* 2}^{2}=A_{* 3}^{2}=-3$ and $z_{* 1}=z_{* 2}=1, z_{* 3}=2$, then the weighted dual graph $\Gamma$ of the exceptional set is one of the following forms.

(1)

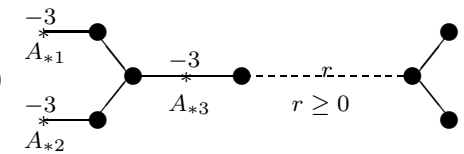

(2)

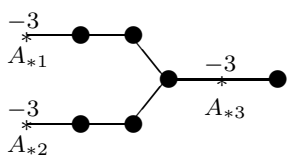

(3)

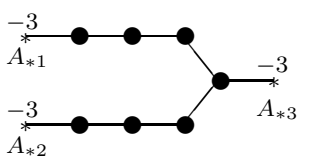

(4)

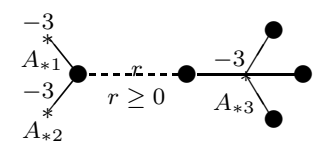

(5)

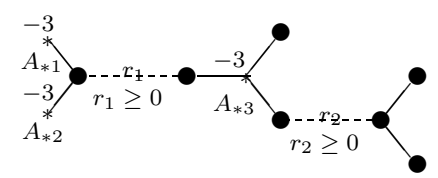

(6)

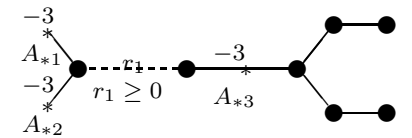

(7)

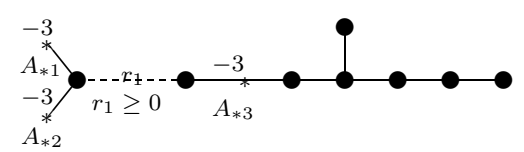

(8)

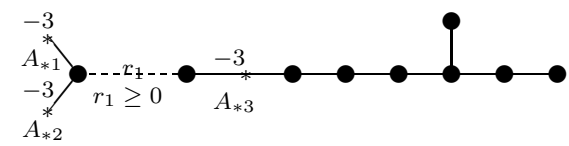

(9)

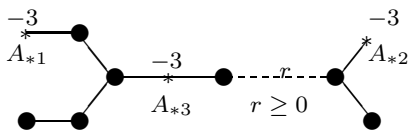

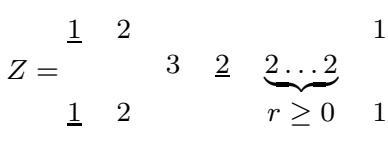

$$
\begin{aligned}
& Z=\begin{array}{llllll}
\underline{1} & 2 & 3 & & & \\
\underline{1} & 2 & 3 & & \underline{2} & 1
\end{array} \\
& Z=\begin{array}{llllll}
\underline{1} & 2 & 3 & 4 & & \\
\underline{1} & 2 & 3 & 4 & & \underline{2}
\end{array}
\end{aligned}
$$$$
Z=\underbrace{\underline{1}}_{\underline{1}} \underbrace{2 \ldots 2}_{r \geq 0} \quad \underline{2} \quad \begin{aligned}
& 1 \\
& 1
\end{aligned}
$$

$$
Z=\underline{\underline{1}} \underbrace{2 \ldots 2}_{r_{1} \geq 0} \quad \underline{2} \underbrace{1 \ldots 2}_{r_{2} \geq 1} 1
$$

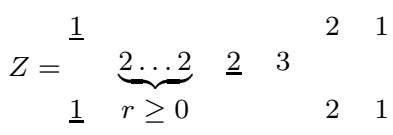$$
Z=\underbrace{1}_{\underline{1}} \underbrace{2 \ldots 2}_{r \geq 0} \quad \underline{2} \quad 3 \quad \begin{array}{lllll}
2 & 4 & 3 & 2 & 1
\end{array}
$$

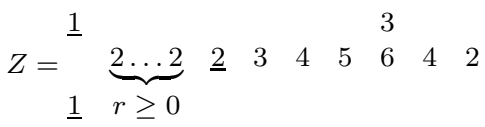

$$
\begin{aligned}
& Z=\underbrace{12}_{12} \underbrace{22 \ldots 2^{1}}_{r \geq 01}
\end{aligned}
$$


(10)

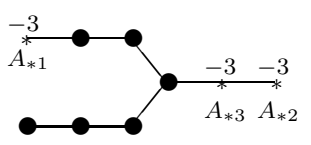

(11)

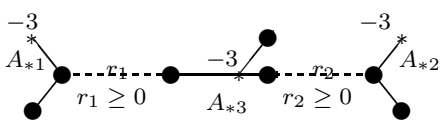

(12)

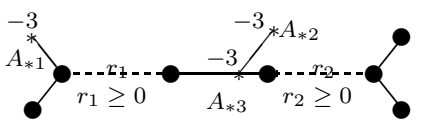

(13)

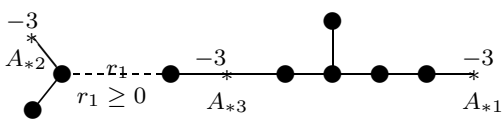

(14)

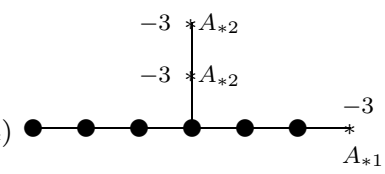

(15)

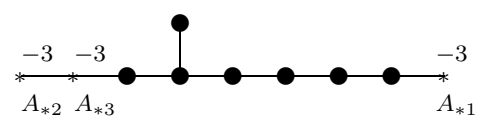

$Z=\begin{array}{llllll}1 & 2 & 3 & & \\ 1 & 2 & 3 & 4 & \underline{2} & 1\end{array}$

$Z=\underline{1} \underbrace{2 \ldots 2}_{r_{1} \geq 0} \quad \underline{2} \underbrace{1 \ldots 2}_{r_{2} \geq 0} \underline{1}$

$Z=\underline{1} \underbrace{2 \ldots 2}_{r_{1} \geq 0} \quad \underline{2} \underbrace{\frac{1}{2} \ldots 2}_{r_{2} \geq 1} 1$

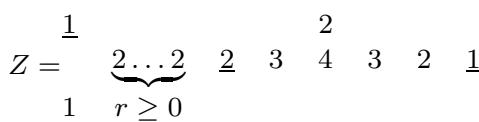

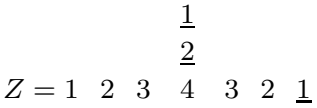

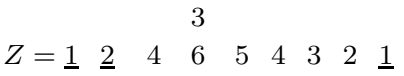

Proof. Observe that $A_{* i} \cdot\left(Z-A_{* i}\right)=2$ for $i=1,2$ and that $A_{* 3} \cdot\left(Z-2 A_{* 3}\right)=$ $2-A_{* 3}^{2}=5$.

Also, by Lemma 6.6 $A_{* 1}$ and $A_{* 2}$ must be of degree one in $Z$. Otherwise Lemma 6.6 says that the $\Gamma$ must be a circular graph and that will force $a_{* 3}=1$, while we assume that $z_{* 3}=2$. It follows that $A_{* 3}$ intersects with every connected component of $\Gamma^{\prime}=\Gamma-\left\{A_{* 1}, A_{* 2}, A_{* 3}\right\}$ and $A_{* i}, i=1,2$, intersects with at most one connected component.

If $A_{* 1}, A_{* 2}$ and $A_{* 3}$ are mutually disjoint and they are all attached to the same connected component of $\Gamma^{\prime}$, by Proposition 6.30 and Proposition 6.31 we are in cases (1)-(8).

If $A_{* 1}, A_{* 2}$ and $A_{* 3}$ are mutually disjoint and they are not all attached to the same connected component of $\Gamma^{\prime}$, then $A_{* 1}$ and $A_{* 3}$ are both attached to a connected component of $\Gamma^{\prime}$, while $A_{* 2}$ and $A_{* 3}$ are both attached to another connected component. By Proposition 6.32 and Proposition 6.31 we are in case (9), (11) and (13).

If $A_{* 1} \cdot A_{* 3}=0$ and $A_{* 2} \cdot A_{* 3}=1$, then $\Gamma^{\prime}$ has only one connected component where $A_{* 1}$ and $A_{* 3}$ are both attached and $A_{* 2}$ does not intersect with any connected component of $\Gamma^{\prime}$. By Proposition 6.32 and Proposition 6.31, we are in case (9) with 
$r=0$, case (10), case (11) with $r_{2}=0$, case (12), case (13) with $r=0$, case (14) and case (15).

If $A_{* i} \cdot A_{* 3}=1, i=1,2$, then every connected component of $\Gamma^{\prime}$ must only intersect with $A_{* 3}$. By Proposition 6.31 we are in case (4) with $r=0$, case (5) with $r_{1}=0$, case (6), (7), (8) with $r=0$, case (11) with $r_{1}=r_{2}=0$ and case (12) with $r_{1}=0$.

Proposition 6.34. Let $\Gamma$ be the minimal resolution graph of a minimally elliptic singularity with fundamental cycle $Z$. Let $\Gamma^{\prime}$ be the subgraph of $\Gamma$ obtained by removing all the effective components of $\Gamma$. Let $A_{* 1}, A_{* 2}$ and $A_{* 3}$ be three effective components of $\Gamma$. Suppose that $\Gamma_{1}$ is a connected component of $\Gamma^{\prime}$ which corresponds to a rational double point graph in Theorem 4.2. Suppose also that $\Gamma_{1}$ intersects with $A_{* 1}, A_{* 2}, A_{* 3}$, but is disjoint from other effective components. Let $Z_{1}$ be the fundamental cycle on $\Gamma_{1}$. Suppose that $A_{* 1} \cdot Z_{1}=1=A_{* 2} \cdot Z_{1}=A_{* 3} \cdot Z_{1}$. If $A_{* 1}$, $A_{* 2}$, and $A_{* 3}$ are mutually disjoint, $z_{* 1}=1=z_{* 2}=z_{* 3}$, and $A_{* 1}^{2} \leq-3, A_{* 2}^{2} \leq-3$, $A_{* 3}^{2} \leq-3$, then $A_{* 1} \cup A_{* 2} \cup A_{* 3} \cup \Gamma_{1}$ and the restriction of $Z$ on $A_{* 1} \cup A_{* 2} \cup A_{* 3} \cup \Gamma_{1}$ must be one of the following forms.

(1)

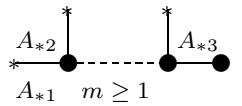

(2)

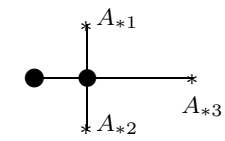

(3)

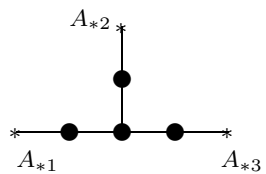

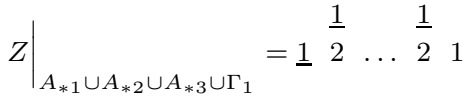

$\left.Z\right|_{A_{* 1} \cup A_{* 2} \cup A_{* 3} \cup \Gamma_{1}}=1 \frac{\underline{1}}{2} \underline{1}$

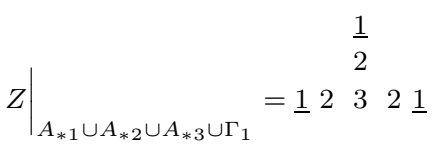

Proof. The proof is the same as that in Proposition 6.30.

Theorem 6.35. Let $(V, p)$ be a germ of minimally elliptic singularity. Let $\pi: M \rightarrow$ $V$ be the minimal resolution of $p$. If case (10) of Proposition 6.2 holds, i.e., if there exist three effective components $A_{* 1}, A_{* 2}$ and $A_{* 3}$ with $A_{* 1}^{2}=A_{* 2}^{2}=-3, A_{* 3}^{2}=-4$ and $z_{* 1}=z_{* 2}=z_{* 3}=1$, then the weighted dual graph $\Gamma$ of the exceptional set is one of the following forms.

(1)

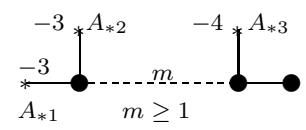

$(2)$

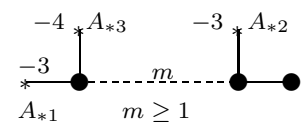

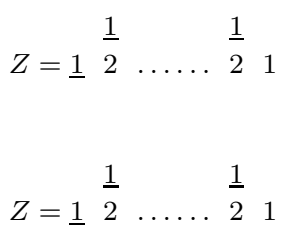


(3)

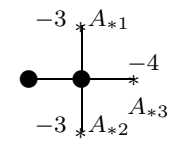

(4)

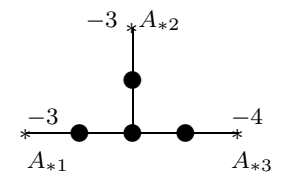

(5)

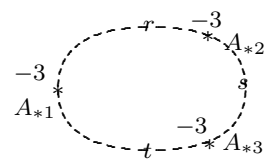

$$
\begin{aligned}
& Z=1 \stackrel{\underline{1}}{2} \underline{1} \\
& 1
\end{aligned}
$$

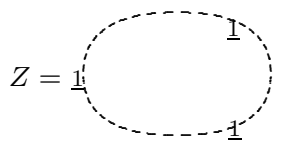

Proof. This follows from Proposition 6.3. Proposition 6.21, Proposition 6.26 and Proposition 6.32

Proposition 6.36. Let $\Gamma$ be the minimal resolution graph of a minimally elliptic singularity with fundamental cycle $Z$. Let $\Gamma^{\prime}$ be the subgraph of $\Gamma$ obtained by removing all the effective components of $\Gamma$. Let $A_{* 1}, A_{* 2}, A_{* 3}$ and $A_{* 4}$ be four effective components of $\Gamma$. Suppose that $\Gamma_{1}$ is a connected component of $\Gamma^{\prime}$ which corresponds to a rational double point graph in Theorem 4.2. Suppose also that $\Gamma_{1}$ intersects with $A_{* 1}, A_{* 2}, A_{* 3}$ and $A_{* 4}$, but is disjoint from other effective components. Let $Z_{1}$ be the fundamental cycle on $\Gamma_{1}$. Suppose that $A_{* 1} \cdot Z_{1}=1=$ $A_{* 2} \cdot Z_{1}=A_{* 3} \cdot Z_{1}=A_{* 4} \cdot Z_{1}$. If $A_{* 1}, A_{* 2}, A_{* 3}$ and $A_{* 4}$ are mutually disjoint, $z_{* 1}=z_{* 2}=z_{* 3}=z_{* 4}=1$, and $A_{* 1}^{2} \leq-3, A_{* 2}^{2} \leq-3, A_{* 3}^{2} \leq-3, A_{* 4}^{2} \leq-3$, then $A_{* 1} \cup A_{* 2} \cup A_{* 3} \cup A_{* 4} \cup \Gamma_{1}$ and the restriction of $Z$ on $A_{* 1} \cup A_{* 2} \cup A_{* 3} \cup A_{* 4} \cup \Gamma_{1}$ must be one of the following forms.

(1)

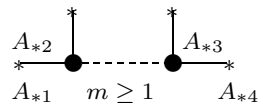

(2)

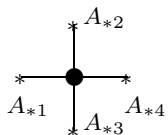

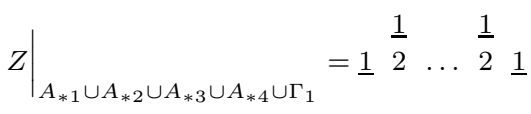

$\left.Z\right|_{A_{* 1} \cup A_{* 2} \cup A_{* 3} \cup A_{* 4} \cup \Gamma_{1}}=\underline{1} \begin{aligned} & \underline{1} \\ & \underline{1}\end{aligned}$

Proof. The proof is the same as that in Proposition 6.32.

Theorem 6.37. Let $(V, p)$ be a germ of minimally elliptic singularity. Let $\pi$ : $M \longrightarrow V$ be the minimal resolution of $p$. If case (11) of Proposition 6.2 holds, i.e., if there exist four effective components $A_{* 1}, A_{* 2}, A_{* 3}$ and $A_{* 4}$ with $A_{* 1}^{2}=-3=$ $A_{* 2}^{2}=A_{* 3}^{2}=A_{* 4}^{2}$ and $z_{* 1}=1=z_{* 2}=z_{* 3}=z_{* 4}$, then the weighted dual graph $\Gamma$ of the exceptional set is one of the following forms. 
(1)

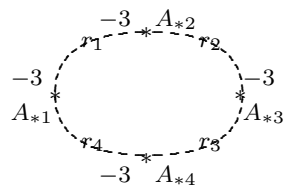

$(2)$

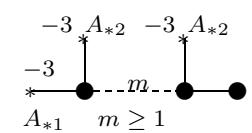

(3)

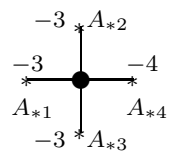

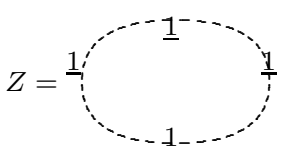

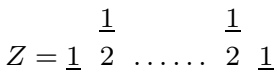

$Z=\underline{1} \begin{array}{ll}\underline{1} & \\ 2 & 1\end{array}$

Proof. This follows from Proposition 6.3, Proposition 6.21, Proposition 6.26, Proposition 6.32 and Proposition 6.34.

\section{Complete List of Weighted dual GRAphS OF Minimally ELliPtic SINGULARITIES WITH $Z^{2}=-4$}

In the following, we shall list all the weighted dual graphs of minimally elliptic singularities with $Z^{2}=-4$ according to Proposition 6.2 Before we do this, we shall adopt the following notation, some of which was used by Laufer La4. The special cases of Proposition 3.7, where it is not true that the $A_{i}$ are nonsingular rational curves with normal crossings, are described and named individually.

(1) with $r$ vertices and $r+1$ edges.

The case $r=0$ is included.

- is a nonsingular rational curve with weight -2 .

(2) $E_{\ell} \quad *$ The vertex $A_{*}$ is a nonsingular elliptic curve.

(3) $N_{0}$ (a) $\quad *$ The vertex $A_{*}$ is a rational curve with a node singularity.

(b)

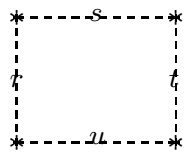

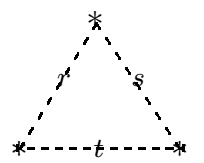
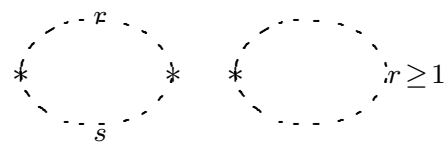

Each $A_{*}$ is a nonsingular rational curve.

(4) $C_{u}$

$*$

(5) $T_{a}$

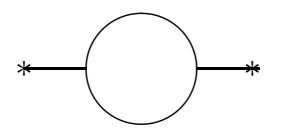

The two $A_{*}$ are nonsingular rational curves which meet tangentially to first order.

(6) $T_{r}$

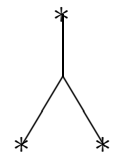

The three $A_{*}$ are nonsingular rational curves which meet transversely at the same point. 
(7) $A_{*, m, n}$

(a) $A$

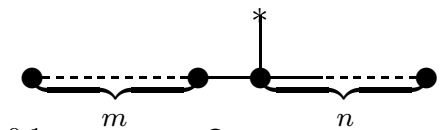

$A_{*, 0,2}$

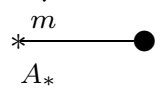

$A_{*}$

$A_{*, 0,3}$

(b) $A_{*, 1,2}$

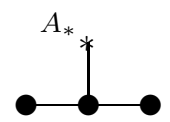

$A_{*, 1,4}$

$$
A
$$

$A_{*, 1,6}$

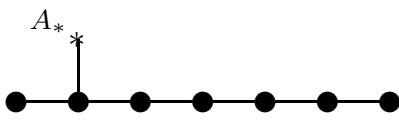

(c) $A_{*, 2,3}$
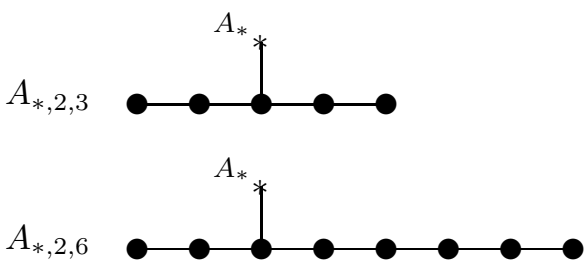

$$
A_{*}
$$

$A_{*, 2,9}$

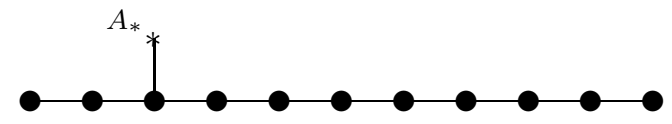

(d) $A_{*, 3,4}$
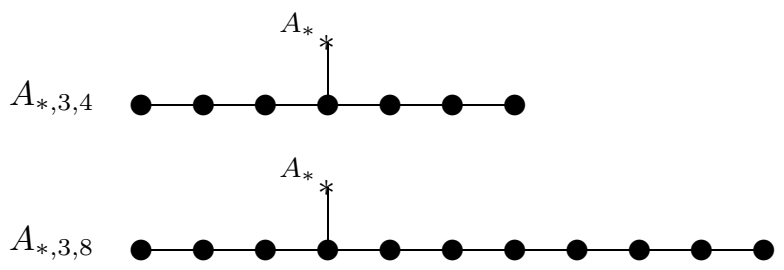

$A_{*}$

(e) $A_{*, 4,5}$

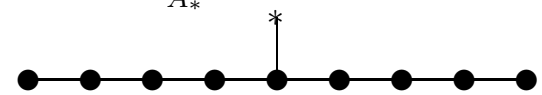

$A_{*}$

(f) $A_{*, 5,6}$

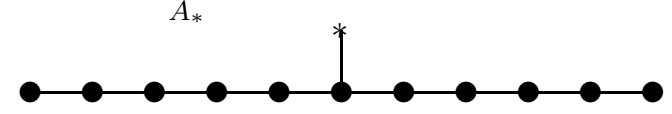

(8) $A_{* *, n, m, \ell}$

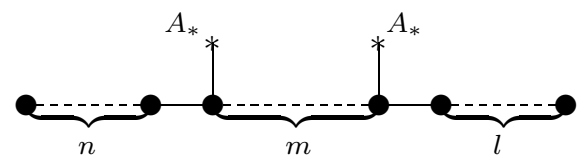


(a) $A_{* *, 0,1,2}$

$A_{* 1} \quad A_{* 2}$

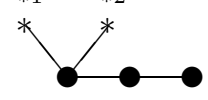

$A_{* *, 0,1,1}$

$A_{* 1} \quad A_{* 2}$

.

$A_{* 1} \quad A_{* 2}$

$A_{* *, 1,1,1}$

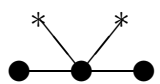

$A_{* 1} \quad A_{* 2}$

(b) $A_{* *, 3,2,0}$

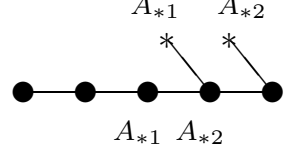

$A_{* *, 2,2,0}$

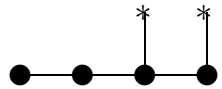

(c) $A_{* *, 1,3,0}$
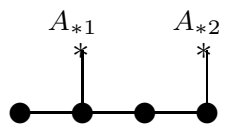

$A_{* *, 3,3,0}$

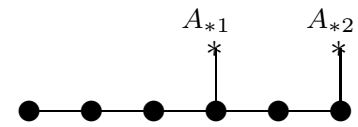

$A_{* *, 4,3,0}$
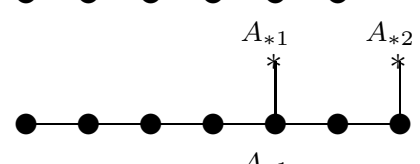

(d) $A_{* *, 4,4,0}$

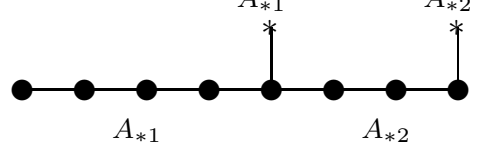

(e) $A_{* *, 2,5,0}$

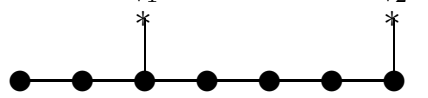

(f) $A_{* *, 3,7,0}$

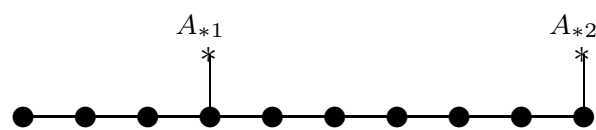

(g) $A_{* *, 0, m, 0}$
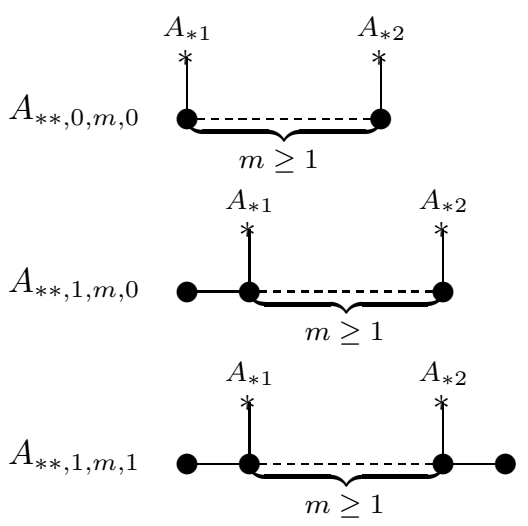
(9) $A_{* * *, m, n, k}$

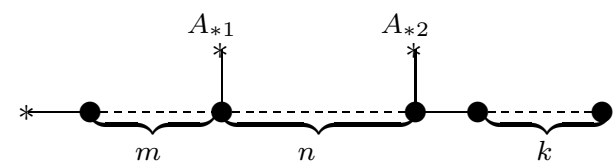

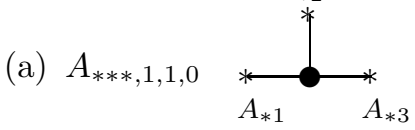

(b) $A_{* * *, 1, n, 0}$

(c) $A_{* *, 2,2,0}$

(d) $A_{* * *, 3,3,0}$

(e) $A_{* *, 4,4,0}$

(f) $A_{* * *, 1, n, 1}$

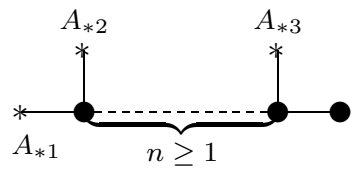

(10) $A_{* * * *, m}$

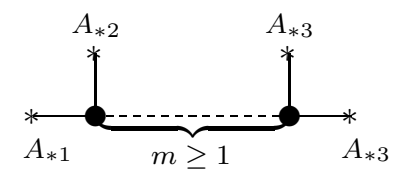

(11) $D_{m, *}$

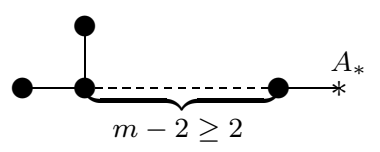

(12)

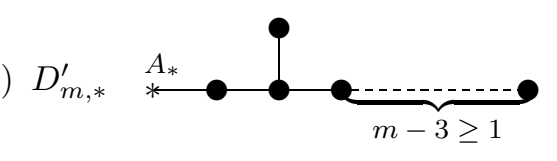

(a) $D_{5, *}^{\prime} \stackrel{A_{*}}{\longrightarrow} \longrightarrow$

(b) $D_{6, *}^{\prime}$ 
(c) $D_{7, *}^{\prime} \quad A_{*} \bullet$

(d) $D_{8, *}^{\prime} \quad A_{*} \bullet$
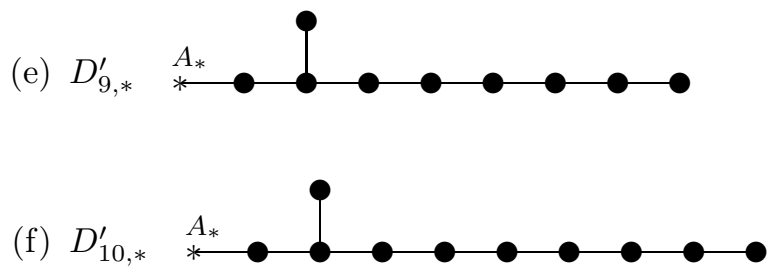

(g) $D_{11, *}^{\prime}$

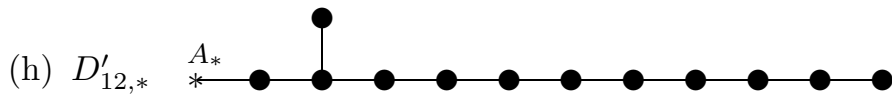

(13) $D_{m, *}^{\prime \prime}$

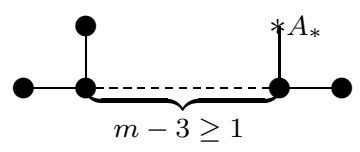

(14) $D_{m, * *}$

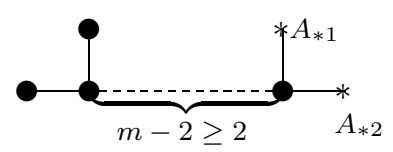

(15) $D_{5, * *}^{\prime}$

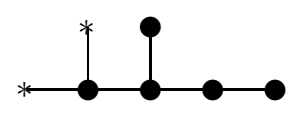

(16) (a) $D_{4, * *}^{\prime \prime}$

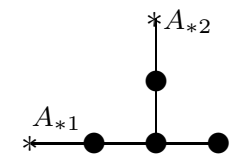

(b) $D_{5, * *}^{\prime \prime}$

(17) (a) $D_{5, * *}^{\prime \prime \prime}$

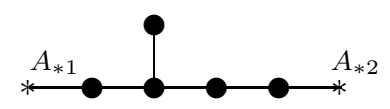


(b) $D_{6, * *}^{\prime \prime \prime}$

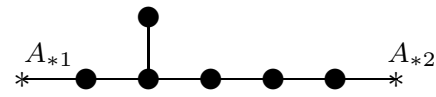

(c) $D_{7, * *}^{\prime \prime \prime}$

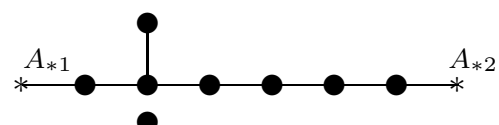

(d) $D_{8, * *}^{\prime \prime \prime}$

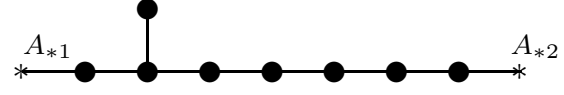

(e) $D_{9, * *}^{\prime \prime \prime}$

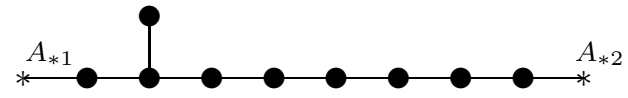

(18)

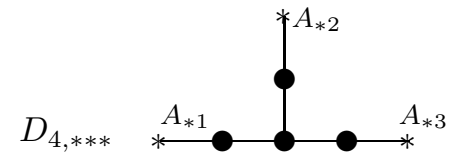

(19)

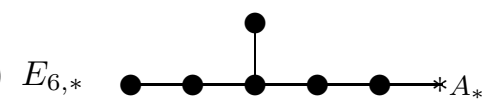

(20)

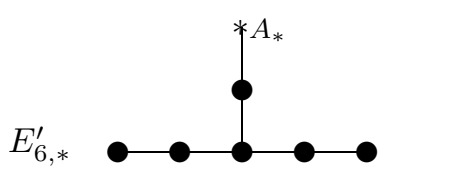

(21)

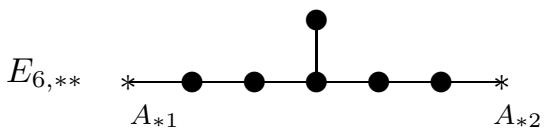

(22)

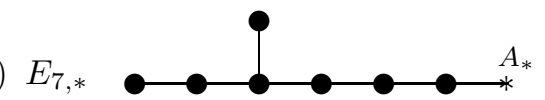

(23)
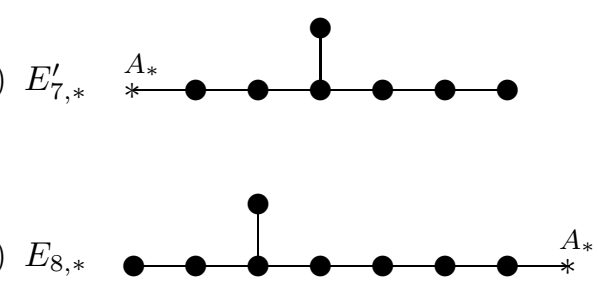

In the weighted dual graphs in the following tables, we may use $*^{\prime}$ or o to replace the $*$ in graphs (1)-(24) above if it is necessary. Except in part I of the tables, at the beginning of each part of the tables, we will list values of $A_{*} \cdot A_{*}, A *^{\prime} \cdot A_{*^{\prime}}$, $A_{o} \cdot A_{o}, z_{*}, z_{*^{\prime}}$, and $z_{o}$ when they are used in the dual graphs of that part.

Example 1. At the beginning of Table V, we give the values $A_{*} \cdot A_{*}=A_{*^{\prime}} \cdot A_{*^{\prime}}=-3$ and $z_{*}=z_{*^{\prime}}=2$. Therefore the notation $A_{*, 0,1}+A_{*, 0,1}+A_{*, *^{\prime}, 0,1,2}+A_{*^{\prime}, 0,1}+A_{*^{\prime}, 0,1}$ denotes the weighted dual graph 

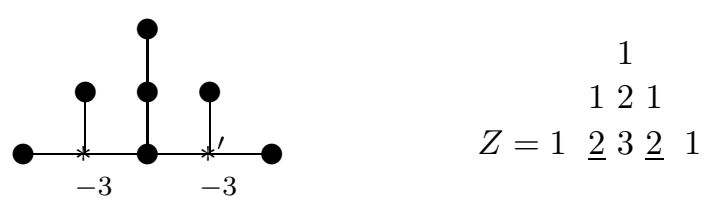

Example 2. At the beginning of Table VII, we give $A_{*} \cdot A_{*}=-4, A_{*^{\prime}} \cdot A_{*^{\prime}}=-3$, $z_{*}=1$ and $z_{*^{\prime}}=2$. The notation $A_{*, *^{\prime}, m, 0}+A_{*^{\prime}, 0,1}+A_{*^{\prime}, 0,1}+A_{*^{\prime}, 0,1}$ denotes the graph

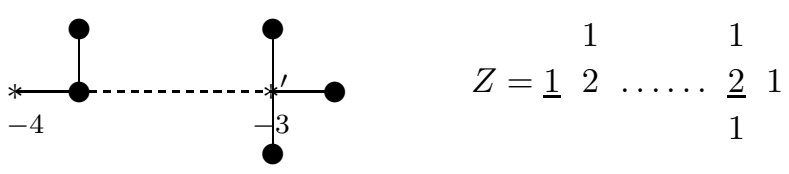

Example 3. At the beginning of Table X, we give $A_{*} \cdot A_{*}=A_{*^{\prime}} \cdot A_{*^{\prime}}=A_{\mathbf{o}} \cdot A_{\mathbf{o}}=-3$ $z_{*}=z_{*^{\prime}}=1$ and $z_{\mathbf{o}}=2$. The notation $A_{*, \mathbf{o}, 0,0,0}+A_{\mathbf{o}, 0,1}+A_{\mathbf{o}, *^{\prime}, 1,1,0}$ denotes the graph

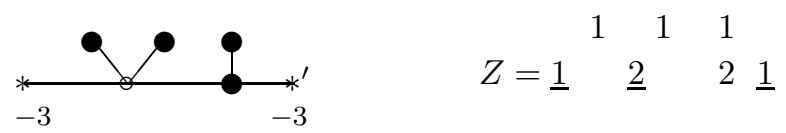

\section{TABLE}

The weighted dual graphs for minimally elliptic singularities with $Z \cdot Z=-4$.

I. The following graphs correspond to those exceptional cases in Proposition 3.7

$A_{*} \cdot A_{*}$

1. $E_{\ell}$

2. $N_{0}$

3. $C_{u}$

4. $T_{a}$

5. $T_{r}$

6. $T_{a}$

7. $T_{r}$

8. $T_{a}$

9. $T_{r}$

10. $T_{r}$

$$
\begin{aligned}
& -4 \\
& -4 \\
& -4 \\
& -2,-6 \\
& -2,-2,-6 \\
& -3,-5 \\
& -2,-3,-5 \\
& -4,-4 \\
& -2,-4,-4 \\
& -3,-3,-4
\end{aligned}
$$

II. The following graphs correspond to those in Theorem 6.12
$A_{*} \cdot A_{*}=-3, z_{*}=4$.
1. $A_{*, 0,1}+A_{*, 0,3}+A_{*, 0,3}+A_{*, 0,3}$
2. $A_{*, 0,1}+A_{*, 0,3}+A_{*, 1,6}$
3. $A_{*, 0,1}+A_{*, 2,9}$
4. $A_{*, 0,3}+A_{*, 0,3}+D_{9, *}^{\prime}$
5. $D_{5, *}^{\prime}+A_{*, 1,6}$ 
III. The following graphs correspond to those in Theorem 6.14

$A_{*} \cdot A_{*}=-4, z_{*}=2$.

1. $A_{*, 0,1}+A_{*, 0,1}+A_{*, 0,1}+A_{*, 0,1}+A_{*, 0,1}+A_{*, 0,1}$

2. $A_{*, 0,1}+A_{*, 0,1}+A_{*, 0,1}+A_{*, 0,1}+A_{*, 1,2}$

3. $A_{*, 0,1}+A_{*, 0,1}+A_{*, 0,1}+A_{*, 0,1}+D_{m, *}$

4. $A_{*, 0,1}+A_{*, 0,1}+A_{*, 0,1}+A_{*, 2,3}$

5. $A_{*, 0,1}+A_{*, 0,1}+A_{*, 0,1}+D_{6, *}^{\prime 2,}$

6. $A_{*, 0,1}+A_{*, 0,1}+A_{*, 0,1}+E_{7, *}$

7. $A_{*, 1,2}+A_{*, 0,1}+A_{*, 0,1}+A_{*, 1,2}$

8. $D_{r, *}+A_{*, 0,1}+A_{*, 0,1}+A_{*, 1,2}$

9. $D_{r, *}+A_{*, 0,1}+A_{*, 0,1}+D_{s, *}$

10. $A_{*, 0,1}+A_{*, 0,1}+D_{8, *}^{\prime}$

11. $A_{*, 0,1}+A_{*, 0,1}+A_{*, 3,4}$

12. $A_{*, 1,2}+A_{*, 0,1}+A_{*, 2,3}$

13. $D_{m, *}+A_{*, 0,1}+A_{*, 2,3}$

14. $A_{*, 1,2}+A_{*, 0,1}+D_{6, *}^{\prime}$

15. $D_{m, *}+A_{* 0,1}+D_{6, *}^{\prime}$

16. $A_{*, 1,2}+A_{*, 0,1}^{\prime}+E_{7, *}$

17. $D_{m, *}+A_{*, 0,1}+E_{7, *}$

18. $A_{*, 0,1}+A_{*, 4,5}$

19. $A_{*, 0,1}+D_{10, *}^{\prime}$

20. $A_{*, 1,2}+A_{*, 1,2}+A_{*, 1,2}$

21. $A_{*, 1,2}+A_{* 1,2}+D_{m, *}$

22. $A_{*, 1,2}+D_{m, *}+D_{n, *}$

23. $D_{m, *}+D_{n, *}+D_{k, *}$

24. $A_{*, 1,2}+A_{*, 3,4}$

25. $D_{m, *}+A_{*, 3,4}$

26. $A_{*, 1,2}+D_{8, *}^{\prime}$

27. $D_{m, *}+D_{8, *}^{\prime}$

28. $A_{*, 2,3}+A_{*, 2,3}$

29. $A_{*, 2,3}+D_{6, *}^{\prime}$

30. $A_{*, 2,3}+E_{7, *}$

31. $D_{6, *}^{\prime}+D_{6, *}^{\prime}$

32. $D_{6, *}^{\prime}+E_{7, *}$

33. $E_{7, *}+E_{7, *}$

34. $A_{*, 5,6}$

35. $D_{12, *}^{\prime}$

IV. The following graphs correspond to those in Theorem 6.16 . $A_{*} \cdot A_{*}=-6, z_{*}=1$.

1. $N o, r \geq 1$

2. $D_{m, *}^{\prime \prime}$

3. $E_{6, *}^{\prime}$

4. $E_{7, *}^{\prime}$

5. $E_{8, *}$ 
V. The following graphs correspond to those in Theorem 6.19,

$A_{*} \cdot A_{*}=A_{*^{\prime}} \cdot A_{*^{\prime}}=-3, z_{*}=z_{*^{\prime}}=2$.

1. $A_{*, 0,1}+A_{*, 0,1}+A_{*, *^{\prime}, 0,1,2}+A_{*^{\prime}, 0,1}+A_{*^{\prime}, 0,1}$

2. $A_{*, 0,1}+A_{*, 0,1}+A_{*, *^{\prime}, 0,1,2}+A_{*^{\prime}, 1,2}$

3. $A_{*, 0,1}+A_{*, 0,1}+A_{*, *^{\prime}, 0,1,2}+D_{m, *^{\prime}}^{\prime}$

4. $A_{*, 1,2}+A_{*, *^{\prime}, 0,1,2}+A_{*^{\prime}, 1,2}$

5. $A_{*, 1,2}+A_{*, *^{\prime}, 0,1,2}+D_{m, *^{\prime}}$

6. $D_{m, *}+A_{*, *^{\prime}, 0,1,2}+D_{n, *^{\prime}}$

7. $A_{*, 0,1}+A_{*, 0,1}+A_{*, 0,1}+A_{*, *^{\prime}, 0, m, 0}+A_{*^{\prime}, 0,1}+A_{*^{\prime}, 0,1}+A_{*^{\prime}, 0,1}$

8. $A_{*, 0,1}+A_{*, 0,1}+A_{*, 0,1}+A_{*, *^{\prime}, 0, m, 0}+A_{*^{\prime}, 0,1}+A_{*^{\prime}, 1,2}$

9. $A_{*, 0,1}+A_{*, 0,1}+A_{*, 0,1}+A_{*, *^{\prime}, 0, m, 0}+A_{*^{\prime}, 0,1}+D_{m, *^{\prime}}$

10. $A_{*, 0,1}+A_{* .0,1}+A_{*, 0,1}+A_{*, *^{\prime}, 0, m, 0}+A_{*^{\prime}, 2,3}$

11. $A_{*, 0,1}+A_{* .0,1}+A_{*, 0,1}+A_{*, *^{\prime}, 0, m, 0}+D_{6, *^{\prime}}^{\prime}$

12. $A_{*, 0,1}+A_{* .0,1}+A_{*, 0,1}+A_{*, *^{\prime}, 0, m, 0}+E_{7, *^{\prime}}$

13. $A_{*, 1,2}+A_{*, 0,1}+A_{*, *^{\prime}, 0, m, 0}+A_{*^{\prime}, 0,1}+A_{*^{\prime}, 1,2}$

14. $D_{n, *}+A_{*, 0,1}+A_{*, *^{\prime}, 0, m, 0}+A_{*^{\prime}, 0,1}+A_{*^{\prime}, 1,2}$

15. $D_{n, *}+A_{*, 0,1}+A_{*, *^{\prime}, 0, m, 0}+A_{*^{\prime}, 0,1}+D_{k, *^{\prime}}$

16. $A_{*, 1,2}+A_{*, 0,1}+A_{*, *^{\prime}, 0, m, 0}+A_{*^{\prime}, 2,3}$

17. $D_{n, *}+A_{*, 0,1}+A_{*, *^{\prime}, 0, m, 0}+A_{*^{\prime}, 2,3}$

18. $A_{*, 1,2}+A_{*, 0,1}+A_{*, *^{\prime}, 0, m, 0}+D_{6, *^{\prime}}^{\prime}$

19. $D_{n, *}+A_{*, 0,1}+A_{*, *^{\prime}, 0, m, 0}+D_{6, *^{\prime}}^{\prime}$

20. $A_{*, 1,2}+A_{*, 0,1}+A_{*, *^{\prime}, 0, m, 0}+E_{7, *^{\prime}}$

21. $D_{n, *}+A_{*, 0,1}+A_{*, *^{\prime}, 0, m, 0}+E_{7, *^{\prime}}$

22. $A_{*, 2,3}+A_{*, *^{\prime}, 0, m, 0}+A_{*^{\prime}, 2,3}$

23. $A_{*, 2,3}+A_{*, *^{\prime}, 0, m, 0}+D_{6, *^{\prime}}^{\prime}$

24. $A_{*, 2,3}+A_{*, *^{\prime}, 0, m, 0}+E_{7, *^{\prime}}$

25. $D_{6, *}^{\prime}+A_{*, *^{\prime}, 0, m, 0}+D_{6, *^{\prime}}^{\prime}$

26. $D_{6, *}^{\prime}+A_{*, *^{\prime}, 0, m, 0}+E_{7, *^{\prime}}$

27. $E_{7, *}+A_{*, *^{\prime}, 0, m, 0}+E_{7, *^{\prime}}$

28. $A_{*, 0,1}+A_{*, *^{\prime}, 3,2,0}+A_{*^{\prime}, 0,1}+A_{*^{\prime}, 0,1}$

29. $A_{*, 0,1}+A_{*, *^{\prime}, 3,2,0}+A_{*^{\prime}, 1,2}$

30. $A_{*, 0,1}+A_{*, *^{\prime}, 3,2,0}+D_{m, *^{\prime}}$

31. $A_{*, *^{\prime}, 4,3,0}+A_{*^{\prime}, 0,1}+A_{*^{\prime}, 0,1}$

32. $A_{*, *^{\prime}, 4,3,0}+A_{*^{\prime}, 1,2}$

33. $A_{*, *^{\prime}, 4,3,0}+D_{m, *^{\prime}}$

34. $A_{*, 0,1}+A_{*, 0,1}+D_{4, *, *^{\prime}}^{\prime \prime}+A_{*^{\prime}, 0,1}+A_{*^{\prime}, 0,1}$

35. $A_{*, 0,1}+A_{*, 0,1}+D_{4, *, *^{\prime}}^{\prime \prime}+D_{m, *^{\prime}}$

36. $A_{*, 0,1}+A_{*, 0,1}+D_{4, *, *^{\prime}}^{\prime \prime}+A_{*^{\prime}, 1,2}$

37. $D_{m, *}+D_{4, *, *^{\prime}}^{\prime \prime}+A_{*^{\prime}, 1,2}$

38. $D_{m, *}+D_{4, *, *^{\prime}}^{\prime \prime}+D_{n, *^{\prime}}$

39. $A_{*^{\prime}, 1,2}+D_{4}^{\prime \prime}$

40. $A_{*, 0,1}+D_{6, *, *^{\prime}}^{\prime \prime \prime}+A_{*^{\prime}, 0,1}+A_{*^{\prime}, 0,1}$

41. $A_{*, 0,1}+D_{6, *, *^{\prime}}^{\prime \prime \prime}+A_{*^{\prime}, 1,2}^{\prime \prime \prime}$

42. $A_{*, 0,1}+D_{6, *, *^{\prime}}^{\prime \prime \prime}+D_{n, *^{\prime}}$

43. $D_{8, *, *^{\prime}}^{\prime \prime \prime}+A_{*^{\prime}, 0,1}+A_{*^{\prime}, 0,1}$

44. $D_{8, *, *^{\prime}}^{\prime \prime \prime \prime}+A_{*^{\prime}, 1,2}$

45. $D_{8, *, *^{\prime}}^{\prime \prime \prime \prime}+D_{m, *^{\prime}}$ 
VI. The following graphs correspond to those in Theorem 6.23
$A_{*} \cdot A_{*}=A_{*^{\prime}} \cdot A_{*^{\prime}}=-3, z_{*}=3, z_{*^{\prime}}=1$.
1. $A_{*, 0,2}+A_{*, 0,2}+A_{*, 0,2}+A_{*, *^{\prime}, 0,1,0}$
2. $A_{*, 1,4}+A_{*, 0,2}+A_{*, *^{\prime}, 0,1,0}$
3. $E_{6, *}+A_{*, 0,2}+A_{*, *^{\prime}, 0,1,0}$
4. $A_{*, 2,6}+A_{*, *^{\prime}, 0,1,0}$
5. $A_{*, 0,2}+A_{*, 0,2}+A_{*, *^{\prime}, 1,3,0}$
6. $A_{*, 1,4}+A_{*, *^{\prime}, 1,3,0}$
7. $E_{6, *}+A_{*, *^{\prime}, 1,3,0}$
8. $A_{*, 0,2}+A_{*, *^{\prime}, 2,5,0}$
9. $A_{*, *^{\prime}, 3,7,0}$

VII. The following graphs correspond to those in Theorem 6.25

$A_{*} \cdot A_{*}=-3, A_{*^{\prime}} \cdot A_{*^{\prime}}=-4, z_{*}=2, z_{*^{\prime}}=1$.

1. $A_{*, 0,1}+A_{*, 0,1}+A_{*, 0,1}+A_{*, *^{\prime}, 1,1,0}$

2. $A_{*, 1,2}+A_{*, 0,1}+A_{*, *^{\prime}, 1,1,0}$

3. $D_{n, *}+A_{*, 0,1}+A_{*, *^{\prime}, 1,1,0}$

4. $A_{*, 2,3}+A_{*, *^{\prime}, 1,1,0}$

5. $D_{6, *}^{\prime}+A_{*, *^{\prime}, 1,1,0}$

6. $E_{7, *}+A_{*, *^{\prime}, 1,1,0}$

7. $A_{*, 0,1}+A_{*, 0,1}+A_{*, *^{\prime}, 2,2,0}$

8. $A_{*, 1,2}+A_{*, *^{*}, 2,2,0}$

9. $D_{n, *}+A_{*, *^{\prime}, 2,2,0}$

10. $A_{*, 0,1}+A_{*, *^{\prime}, 3,3,0}$

11. $A_{*, *^{\prime}, 4,4,0}$

12. $A_{*, 0,1}+A_{*, 0,1}+A_{*, 0,1}+A_{*, *^{\prime}, 0, m, 1}$

13. $A_{*, 1,2}+A_{*, 0,1}+A_{*, *^{\prime}, 0, m, 1}$

14. $D_{n, *}+A_{*, 0,1}+A_{*, *^{\prime}, 0, m, 1}$

15. $A_{*, 2,3}+A_{*, *^{\prime}, 0, m, 1}$

16. $D_{6, *}^{\prime}+A_{*, *^{\prime}, 0, m, 1}$

17. $E_{7, *}+A_{*, *^{\prime}, 0, m, 1}$

18. $A_{*, 0,1}+A_{*, 0,1}+D_{5, *, *^{\prime}}^{\prime \prime \prime}$

19. $A_{*, 1,2}+D_{5, *, *^{\prime}}^{\prime \prime \prime}$

20. $D_{n, *}+D_{5, *, *^{\prime}}^{\prime \prime \prime}$

21. $A_{*, 0,1}+D_{7, *, *^{\prime}}^{\prime \prime \prime}$

22. $D_{9, *, *^{\prime}}^{\prime \prime \prime}$

23. $A_{*, 0,1}+A_{*, 0,1}+A_{*, 0,1}+A_{*, 0,1}+A_{*, *^{\prime}, 0,0,0}$

24. $A_{*, 1,2}+A_{*, 0,1}+A_{*, 0,1}+A_{*, *^{\prime}, 0,0,0}$

25. $D_{n, *}+A_{*, 0,1}+A_{*, 0,1}+A_{*, *^{\prime}, 0,0,0}$

26. $A_{*, 2,3}+A_{*, 0,1}+A_{*, *^{\prime}, 0,0,0}$

27. $D_{6, *}^{\prime}+A_{*, 0,1}+A_{*, *^{\prime}, 0,0,0}$

28. $E_{7, *}+A_{*, 0,1}+A_{*, *^{\prime}, 0,0,0}$

29. $A_{*, 1,2}+A_{*, 1,2}+A_{*, *^{\prime}, 0,0,0}$

30. $D_{n, *}+A_{*, 1,2}+A_{*, *^{\prime}, 0,0,0}$

31. $D_{n, *}+D_{m, *}+A_{*, *^{\prime}, 0,0,0}$

32. $A_{*, 3,4}+A_{*, *^{\prime}, 0,0,0}$

33. $D_{8, *}^{\prime}+A_{*, *^{\prime}, 0,0,0}$ 
VIII. The following graphs correspond to those in Theorem 6.27
$A_{*} \cdot A_{*}$
1. $N o$
2. $A_{*, *^{\prime}, 1, m, 1}$
3. $A_{*, *^{\prime}, 1,1,1}$
4. $D_{m, *, *^{\prime}}$
5. $D_{5, *, *^{\prime}}^{\prime \prime}$
6. $E_{6, *, *^{\prime}}$

IX. The following graphs correspond to those in Theorem 6.29 $A_{*} \cdot A_{*}=-3, A_{*^{\prime}} \cdot A_{*^{\prime}}=-5, z_{*}=z_{*^{\prime}}=1$.

1. No

2. $A_{*, *^{\prime}, 1, m, 1}$

3. $D_{m, *, *^{\prime}, m \geq 4}$

4. $D_{5, *, *^{\prime}}^{\prime \prime}$

5. $E_{6, *, *^{\prime}}$

$\mathbf{X}$. The following graphs correspond to those in Theorem 6.33.

$A_{*} \cdot A_{*}=A_{*^{\prime}} \cdot A_{*^{\prime}}=A_{\mathbf{o}} \cdot A_{\mathbf{o}}=-3, z_{*}=z_{*^{\prime}}=1, z_{\mathbf{o}}=2$.

1. $A_{*, \mathbf{o}, *^{\prime}, 2,2,0}+A_{\mathbf{o}, 1,2}$

2. $A_{*, \mathbf{o}, *^{\prime}, 2,2,0}+D_{n, \mathbf{o}}$

3. $A_{*, \mathbf{o}, *^{\prime}, 3,3,0}+A_{\mathbf{o}, 0,1}$

4. $A_{*, \mathbf{o}, *^{\prime}, 4,4,0}$

5. $A_{*, \mathbf{o}, 0,0,0}+A_{*^{\prime}, \mathbf{o}, 0,0,0}+A_{\mathbf{o}, 0,1}+A_{\mathbf{o}, 0,1}+A_{\mathbf{o}, 0,1}$

6. $A_{*, *^{\prime}, \mathbf{o}, 1, n, 0}+A_{\mathbf{o}, 0,1}+A_{\mathbf{o}, 0,1}+A_{\mathbf{o}, 0,1}$

7. $A_{*, \mathbf{o}, 0,0,0}+A_{*^{\prime}, \mathbf{o}, 0,0,0}+A_{\mathbf{o}, 0,1}+A_{\mathbf{o}, 1,2}$

8. $A_{*, \mathbf{o}, 0,0,0}+A_{*^{\prime}, \mathbf{o}, 0,0,0}+A_{\mathbf{o}, 0,1}+D_{n, \mathbf{o}}$

9. $A_{*, \mathbf{o}, *^{\prime}, 1, n, 0}+A_{\mathbf{o}, 0,1}+A_{\mathbf{o}, 1,2}$

10. $A_{*, \mathbf{o}, *^{\prime}, 1, n, 0}+A_{\mathbf{o}, 0,1}+D_{n, \mathbf{o}}$

11. $A_{*, \mathbf{o}, 0,0,0}+A_{*^{\prime}, \mathbf{o}, 0,0,0}+A_{\mathbf{o}, 2,3}$

12. $A_{*, \mathbf{o}, *^{\prime}, 1, n, 0}+A_{\mathbf{o}, 2,3}$

13. $A_{*, \mathbf{o}, 0,0,0}+A_{*^{\prime}, \mathbf{o}, 0,0,0}+D_{6, \mathbf{o}}^{\prime}$

14. $A_{*, \mathbf{o}, *^{\prime}, 1, n, 0}+D_{6, \mathbf{o}}^{\prime}$

15. $A_{*, \mathbf{o}, 0,0,0}+A_{*^{\prime}, \mathbf{o}, 0,0,0}+E_{7, \mathbf{o}}$

16. $A_{*, \mathbf{o}, *^{\prime}, 1, n, 0}+E_{7, \mathbf{o}}$

17. $A_{*, \mathbf{o}, 0,2,2}+A_{*^{\prime}, \mathbf{o}, 0,0,0}+A_{\mathbf{o}, 0,1}$

18. $A_{*, \mathbf{o}, 0,2,2}+A_{\mathbf{o}, *^{\prime}, 0, m, 1}$

19. $A_{*, \mathbf{o}, 0,3,3}+A_{\mathbf{o}, *^{\prime}, 0,0,0}$

20. $A_{*, \mathbf{o}, 0,0,0}+A_{*^{\prime}, \mathbf{o}, 0,0,0}+A_{\mathbf{o}, 0,1}+A_{\mathbf{o}, 0,1}+A_{\mathbf{o}, 0,1}$

21. $A_{*, \mathbf{o}, 0,0,0}+A_{\mathbf{o}, 0,1}+A_{\mathbf{o}, 0,1}+A_{\mathbf{o}, *^{\prime}, 0, m, 1}$

22. $A_{*, \mathbf{o}, 1, m, 0}+A_{\mathbf{o}, 0,1}+A_{\mathbf{o}, *^{\prime}, 0, n, 1}$

23. $A_{*, \mathbf{o}, 0,0,0}+A_{*^{\prime}, \mathbf{o}, 0,0,0}+A_{\mathbf{o}, 0,1}+A_{\mathbf{o}, 1,2}$

24. $A_{*, \mathbf{o}, 0,0,0}+A_{*^{\prime}, \mathbf{o}, 0,0,0}+A_{\mathbf{o}, 0,1}+D_{n, \mathbf{o}}$

25. $A_{*, \mathbf{o}, 1, m, 0}+A_{*^{\prime}, \mathbf{o}, 0,0,0}+A_{\mathbf{o}, 1,2}$

26. $A_{*, \mathbf{o}, 1, m, 0}+A_{*^{\prime}, \mathbf{o}, 0,0,0}+D_{n, \mathbf{o}}$

27. $A_{*, \mathbf{o}, 0,0,0}+A_{\mathbf{o}, 0,1}+D_{5, \mathbf{o}, *^{\prime}}^{\prime \prime \prime}$

28. $A_{*, \mathbf{o}, 1, m, 0}+D_{5, \mathbf{o}, *^{\prime}}^{\prime \prime \prime}$

29. $A_{*^{\prime}, \mathbf{o}, 0,0,0}+A_{\mathbf{o}, *, 3,3,0}$

30. $A_{*^{\prime}, \mathbf{o}, 0,0,0}+D_{7, \mathbf{o}, *}^{\prime \prime \prime}$ 
XI. The following graphs correspond to those in Theorem 6.35

$A_{*} \cdot A_{*}=A_{*^{\prime}} \cdot A_{*^{\prime}}=-3, A_{\mathbf{o}} \cdot A_{\mathbf{o}}=-4, z_{*}=z_{*^{\prime}}=z_{\mathbf{o}}=1$.

1. $A_{*, *^{\prime}, \mathbf{o}, 1, n, 1}, n \geq 1$

2. $A_{*, \mathbf{o}, *^{\prime}, 1, n, 1}, n \geq 1$

3. $D_{4, *, *^{\prime}, \mathbf{o}}$

4. $\mathrm{NO}$

XII. The following graphs correspond to those in Theorem 6.37

$A_{*} \cdot A_{*}=-3, z_{*}=1$ for all four effective components.

1. $N o$

2. $A_{*, *, *, *, m}, m \geq 1$

\section{REFERENCES}

[Ar] M. Artin, On isolated rational singularities of surfaces, Amer. J. Math. 88 (1996), 129136. MR0199191 (33:7340)

[Ba] H. Bass, On the ubiquity of Gorenstein rings, Math. Z. 82 (1963), 8-28. MR0153708 $(27: 3669)$

[Gr] H. Grauert, Über Modifikationen und exzeptionelle analytische Mengen, Math. Ann. 146 (1962), 331-368. MR0137127 (25:583)

[Gr-Ri] H. Grauert and O. Riemenschneider, Verschwindungssätze für analytische Kohomologiegruppen auf komplexen Räumen, Invent. Math. 11 (1970), 263-292. MR0302938 (46:2081)

[HNK] F. Hirzebruch, W. Neumann, and S. Koh, Differentiable manifolds and quadratic forms, Lecture Notes in Pure and Applied Mathematics, vol. 4, Marcel Dekker, New York, 1971. MR0341499 (49:6250)

[La1] H. Laufer, Normal two-dimensional singularities, Annals of Math. Studies, no. 71, Princeton University Press, Princeton, NJ, 1971. MR.0320365 (47:8904)

[La2] - On rational singularities, Amer. J. Math. 94 (1972), 597-608. MR0330500 $(48: 8837)$

[La3] Deformations of resolutions of two-dimensional singularities, Rice University Studies 1 (1973), no. 59, 53-96. MR0367277 (51:3519)

[La4] - On minimally elliptic singularities, Amer. J. Math. 99 (1977), 1257-1295. MR $0568898(58: 27961)$

[Mi] J. Milnor, Singular points of complex hypersurfaces, Ann. Math. Studies, no. 61, Princeton University Press, Princeton, NJ, 1968. MR0239612 (39:969)

[Ne] W. Neumann, A calculus for plumbing applied to the topology of complex surface singularities and degenerating complex curves, Trans. A.M.S. 268 (1981), 299-344. MR632532 (84a:32015)

[Se] J.-P. Serre, Groupes algébriques et corps de classes, Actualités Scientifiques et Industrielles, no. 1264, Hermann, Paris, 1959. MR0103191 (21:1973)

[Wa] P. Wagreich, Elliptic singularities of surfaces, Amer. J. Math. 92 (1970), 419-454. MR.0291170 (45:264)

[Ya1] S. S.-T. Yau, Normal singularities of surfaces, Proc. Sympos. Pure Math. 72 (1978), 195-198. MR.520537 (80f:14020)

[Ya2] - On maximally elliptic singularities, Trans. A.M.S. 257 (1980), 269-329. MR:552260 (80j:32021)

Department of Mathematics, University of California at San Diego, La Jolla, CalIFORNIA 92093-0112

Department of Mathematics, John Tyler Community College, 13101 Jefferson Davis Highway, Chester, Virginia 23831-5316

Department of Mathematics, Statistics, and Computer Science (M/C 249), University of Illinois at Chicago, 851 South Morgan Street, Chicago, Illinois 60607-7045

E-mail address: yau@uic.edu 